\title{
Structure-Property Relationships in Conjugated Donor-Acceptor Molecules Based on Cyanoanthracene: Computational and Experimental Studies
}

\author{
Jui-Hsien Lin, Arumugasamy Elangovan and Tong-Ing Ho* \\ Department of Chemistry, National Taiwan University, Taipei - 106, Taiwan
}

Fax:+886-2-23636359; E-mail: hall@ntu.edu.tw

Supporting Information

Contents

Page \#

1. Complete reference 8

S1

2. Synthetic schemes

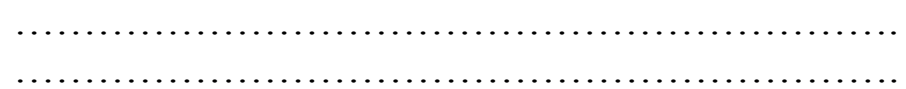

S2-S3

3. Experimental details, characterization data and theoretical method

$\mathrm{S} 4-\mathrm{S} 8$

2. Proton NMR spectra of PANs

S9-S13

3. Proton NMR spectra of PEANs

S14-S18

4. ${ }^{13} \mathrm{C}$ NMR spectra of PANs

$\mathrm{S} 19-\mathrm{S} 23$

5. ${ }^{13} \mathrm{C}$ NMR spectra of PEANs

S24-S28

6. $\mathrm{CV}$ curves of PANs and PEANs

7. Raw ECL spectra

8. Cartesian coordinates for PANs and PEANs.

S35- S51

9. UV-Vis. Solvatochromic spectra of selected compounds

S52

10. Reference

S53

Complete ref. 8(a) Anderson, J. D.; McDonald, E. M.; Lee, P. A.; Anderson, M. L.; Ritchie, E. L.; Hall, H. K.; Hopkins, T.; Mash, E. A.; Wang, J.; Padias, A.; Thayumanavan, S.; Barlow, S.; Marder, S. R.; Jabbour, G. E.; Shaheen, S.; Kippelen, B.; Peyghambarian, N.; Wightman, R. M.; Armstrong, N. R. J. Am. Chem. Soc. 1998, 120, 9646. 
Scheme 1. Synthesis of PANs.

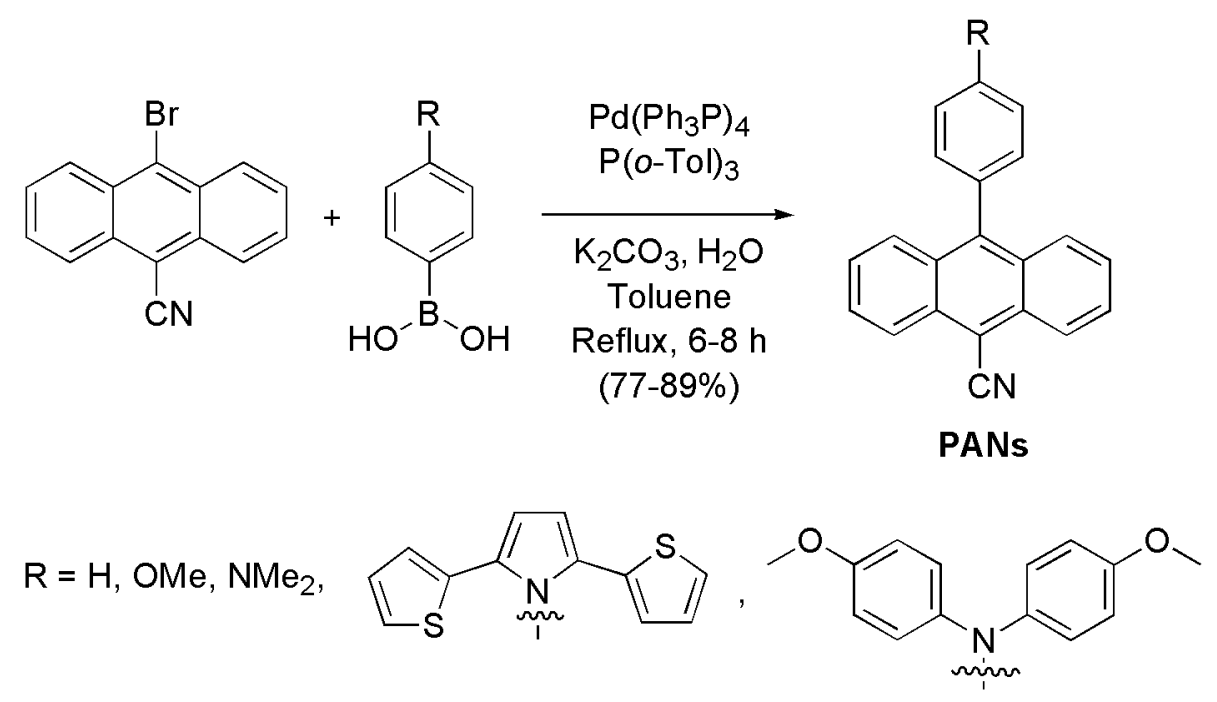

Scheme 2. Synthesis of PEANs.<smiles>[R]c1ccc(C#Cc2c3ccccc3c(CN)c3ccccc23)cc1</smiles><smiles>[R]=CON(C)c1ccc(-c2ccc(-c3cccs3)n2[Al](C)(=O)c2cccs2)cc1</smiles> 
Scheme 3. Synthesis of dithienylpyrrole donors. ${ }^{1}$

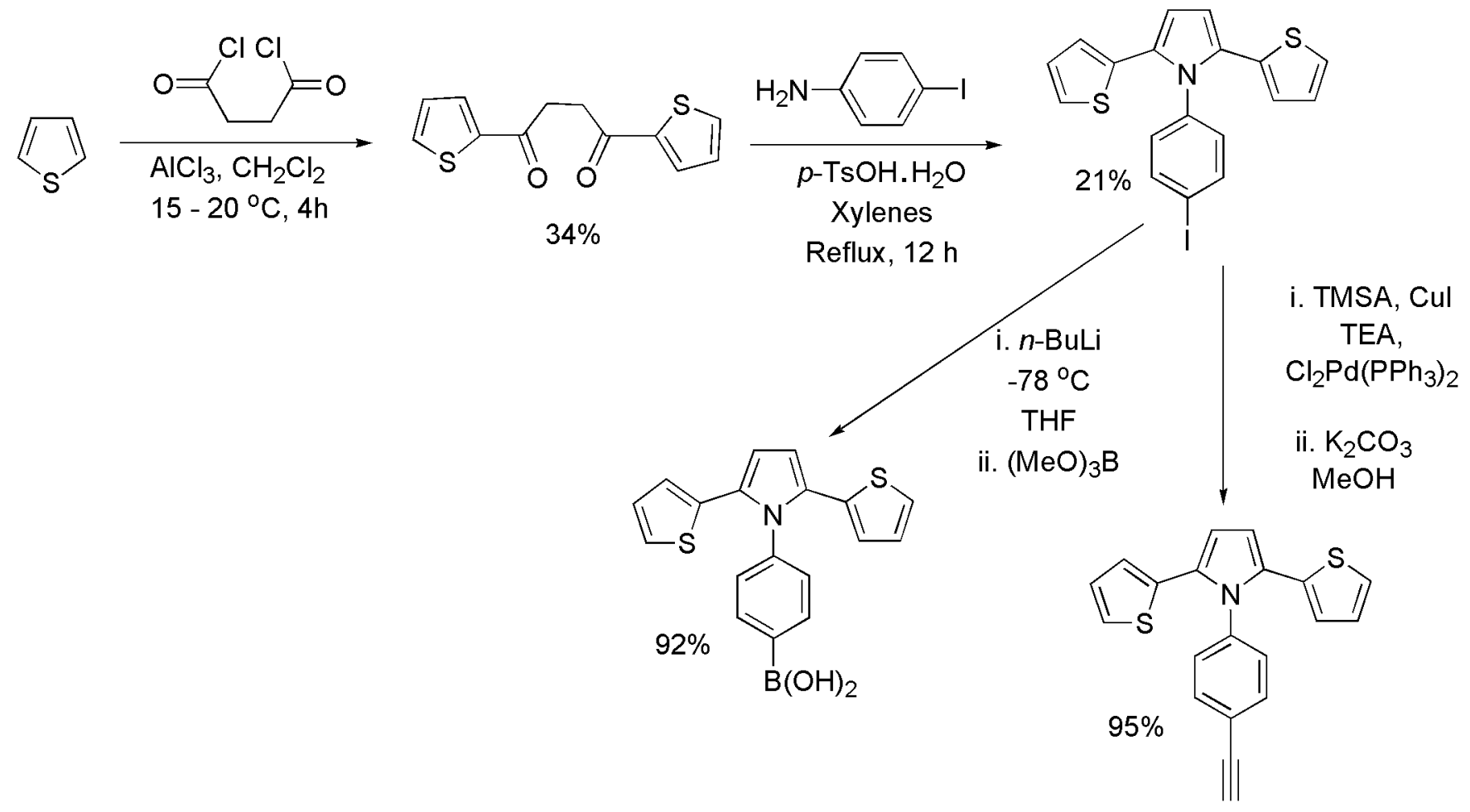

Scheme 4. Synthesis of dianisylaminophenyl donors. ${ }^{2}$

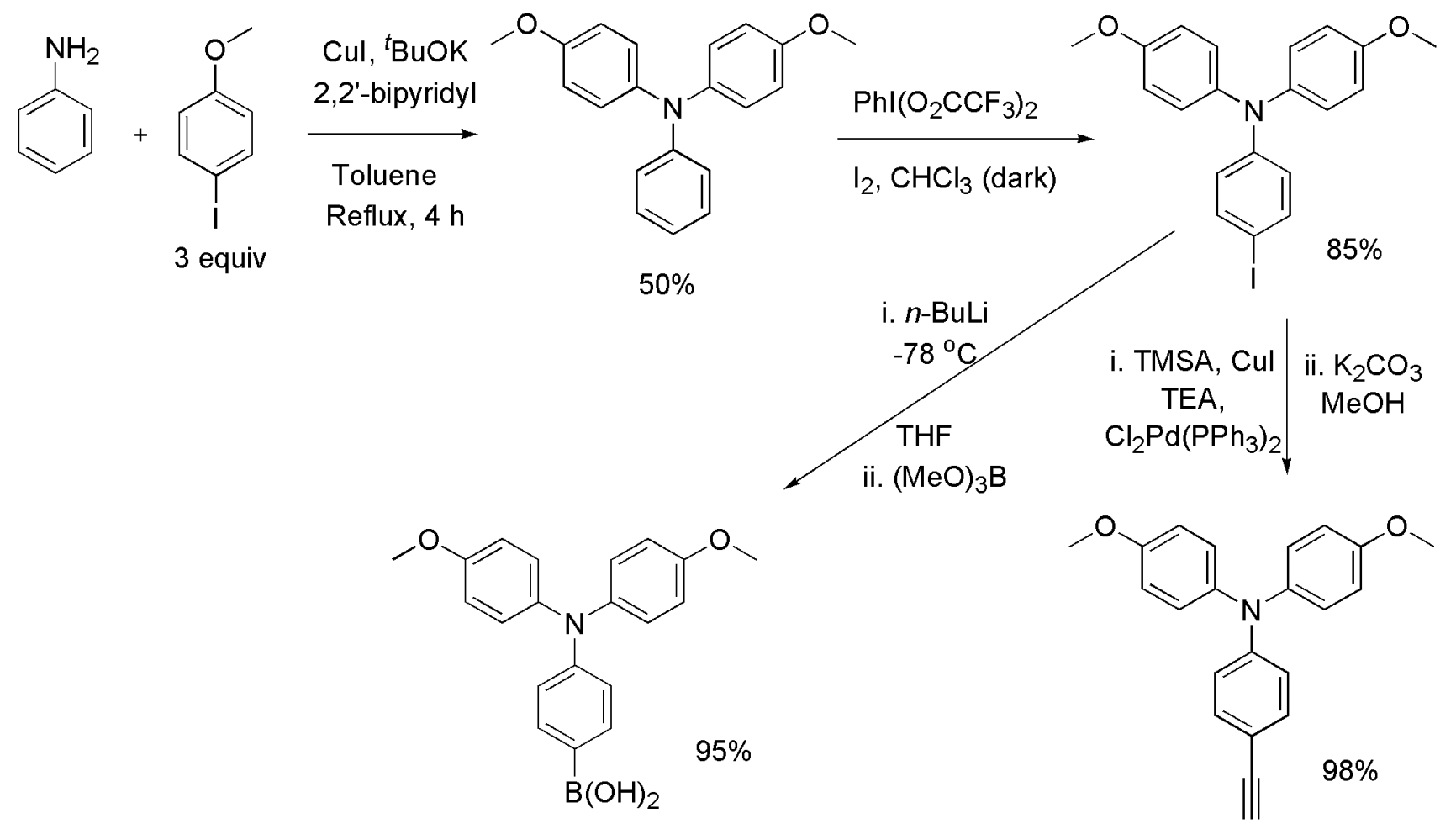




\section{Experimental section}

\section{Materials and methods}

Phenyl acetylene was from a commercial source and was used as received. $p$-Methoxyphenylethyne and $N, N$-dimethylaminophenyethynes were reported earlier. ${ }^{3}$ Phenyl boronic acid was from a commercial source. The high-spin donors, namely the DTP-PE and $\mathbf{A n} \mathbf{n}_{2} \mathbf{N}$-PE were prepared according to the schemes 3 (ref. 9) and 4 (Ref. 12b). Dichlorobis(triphenylphosphine)palladium(II) was either prepared in-house or from commercial source and tetrakis(triphenylphosphine)palladium(0) is from a commercial source. Solvents were distilled as per the standard methods and purged with argon before use. Triethylamine (TEA) and tetrahydrofuran (THF) were distilled and purged with a mixture of approximately 1:1 argon and hydrogen before use. ${ }^{1} \mathrm{H}$ NMR spectra of the samples were recorded with a $400 \mathrm{MHz}$ instrument and ${ }^{13} \mathrm{C}$ NMR spectra were recorded with the same instrument at $100.1 \mathrm{MHz}$ operator frequency in $\mathrm{CDCl}_{3}$ solvent with $\mathrm{CHCl}_{3}$ internal standard $\left(\delta 7.24 \mathrm{ppm}\right.$ for ${ }^{1} \mathrm{H}$ and $77 \mathrm{ppm}$, middle of the three peaks, for ${ }^{13} \mathrm{C}$ spectra. Mass spectra were recorded on nitrobenzylalcohol matrix. TLC was run on precoated aluminium plates ( $\left.\mathrm{Si} 60 \mathrm{~F}_{254}\right)$. Column chromatography was run on silica gel (60-120 mesh) and neutral alumina (70-230 mesh). All UVVisible spectra were recorded on a spectrophotometer with $10 \mu \mathrm{M}$ solution of the compounds and all fluorescence spectra were recorded on fluorescence spectrophotometer using similar solution concentrations in various solvents. Quantum yields were determined using coumarin 334 as standard $(\Phi=0.69$ in $\mathrm{MeOH}) .{ }^{4,5}$

CV measurements were done on an electrochemical analyzer for solutions of the compound in deaerated acetonitrile with a scan rate of $50 \mathrm{mV} / \mathrm{s}$. The cell used was three-electrode cell consisting of carbon disc (2.0 $\mathrm{mm}$ ) working electrode, a platinum wire counter electrode and a $\mathrm{Ag} / \mathrm{AgCl}$ reference electrode. All CV were recorded after calibrating the voltammograph with ferrocene as standard $\left(E_{\mathrm{p} \mathrm{Ox}}=0.5 \mathrm{~V}\right)$. ECL spectra were recorded at room temperatures using a setup consisting of F-3010 Fluorescence spectrophotometer, a voltammograph using Pt wire $(0.25 \mathrm{~mm}$ diameter $)$ and $\mathrm{Pt}$ gauss $(100 \mathrm{mesh}, 0.5 \mathrm{~cm} \times 0.5 \mathrm{~cm})$ electrodes together with a $\mathrm{Ag} / \mathrm{AgCl}$ reference electrode with a computer interface to control the pulsing. The electrode 
surfaces were prepared freshly before CV and ECL experiments. Carbon disc electrode was rubbed against alumina paste followed by rinsing with double-distilled water and $\mathrm{MeCN}$ and wiping with high quality lint free tissue. The Pt wire and the Pt gauss electrodes were cleaned by rinsing with dilute nitric acid followed by water and then they were finally fired with a naked flame to ensure maximum cleanliness of the electrode. A $1 \mathrm{mM}$ concentration of the compound in dry degassed acetonitrile along with $0.05 \mathrm{M}$ tetrabutylammonium perchlorate (TBAP) and the solution was degassed by purging it with dry argon for both CV and ECL measurements. To generate annihilation reaction for ECL, the platinum electrodes were pulsed between first reduction and first oxidation potentials and the pulse interval was controlled on a computer. All measurements were done at room temperatures $\left(22-23{ }^{\circ} \mathrm{C}\right) .^{6}$

General procedure for the synthesis of biaryls. A mixture of the aryl bromide (9-bromo-10cyanoanthracene, prepared by bromination of 9-anthronitrile in nitromethane under reflux for 20 min followed by recrystallization of the precipitate from benzene, $0.1 \mathrm{mmol})$, the boronic acid $(0.11 \mathrm{mmol})$, solid sodium carbonate (3 equiv.) and tetrakis(triphenylphosphine)palladium(0) were degassed and back-filled with argon. To this was added toluene and the mixture was refluxed under stirring for about ten hours when the TLC showed complete disappearance of the starting bromide. The reaction mixture was then quenched with $30 \%$ hydrogen peroxide $(0.5 \mathrm{~mL})$, diluted with water and extracted with ether, dried over magnesium sulphate and the combined organic solution was evaporated. The crude reaction product was purified by column chromatography to get pure coupling products. The following are the characterization data:

9-Cyano-10-phenylanthracene (PAN): Pale lemon yellow crystalline solid, m.p.: $201{ }^{\circ} \mathrm{C} ;{ }^{1} \mathrm{H}$ NMR (400 $\left.\mathrm{MHz}, \mathrm{CDCl}_{3}\right): \delta 8.47(\mathrm{~d}, J=8.8 \mathrm{~Hz}, 2 \mathrm{H}), 7.69(\mathrm{~m}, 4 \mathrm{H}), 7.59(\mathrm{~m}, 3 \mathrm{H}), 7.44(\mathrm{t}, J=6.8 \mathrm{~Hz}, 2 \mathrm{H}), 7.37(\mathrm{~m}, 2 \mathrm{H})$; ${ }^{13} \mathrm{C}$ NMR (100 MHz, $\left.\mathrm{CDCl}_{3}\right): \delta 143.28,136.79,132.64,130.19,129.20,128.21,128.14,127.86,127.44$, 125.82, 125.06, 117.20, 105.34; HR MS (m/z M ): 279.1035, calculated for $\mathrm{C}_{21} \mathrm{H}_{13} \mathrm{~N}: 279.1048$.

9-Anisyl-10-cyanoanthracene (AnAN): Brignt lemon yellow crystalline solid, mp: $230{ }^{\circ} \mathrm{C} ;{ }^{1} \mathrm{H}$ NMR (400 $\left.\mathrm{MHz}, \mathrm{CDCl}_{3}\right): \delta 8.46(\mathrm{~d}, J=8.4 \mathrm{~Hz}, 2 \mathrm{H}), 7.76(\mathrm{~d}, J=9.2 \mathrm{~Hz}, 2 \mathrm{H}), 7.68(\mathrm{t}, J=7.6 \mathrm{~Hz}, 2 \mathrm{H}), 7.44(\mathrm{t}, J=7.6$ $\mathrm{Hz}, 2 \mathrm{H}), 7.29(\mathrm{~d}, J=8.8 \mathrm{~Hz}, 2 \mathrm{H}), 7.12(\mathrm{~d}, J=8.8 \mathrm{~Hz}, 2 \mathrm{H}), 3.95(\mathrm{~s}, 3 \mathrm{H}) ;{ }^{13} \mathrm{C} \mathrm{NMR}(100 \mathrm{MHz}$ 
$\left.\mathrm{CDCl}_{3}\right): \delta 158.44,143.26,137.68,131.40,129.52,128.79,128.17,127.53,125.71,125.04,117.26,113.70$

105.14, 55.56; HR MS (m/z M+ $)$ : 309.1166, calculated for $\mathrm{C}_{22} \mathrm{H}_{15} \mathrm{NO}: 309.1154$.

9- $N, \boldsymbol{N}$-Dimethylanilino-10-cyanoanthracene (DMA-PAN): Orange solid, mp: $>300{ }^{\circ} \mathrm{C} ;{ }^{1} \mathrm{H}$ NMR $(400$ $\left.\mathrm{MHz}, \mathrm{CDCl}_{3}\right): \delta 8.45(\mathrm{~d}, J=8.4 \mathrm{~Hz}, 2 \mathrm{H}), 7.87(\mathrm{~d}, J=9.2 \mathrm{~Hz}, 2 \mathrm{H}), 7.67(\mathrm{t}, J=7.6 \mathrm{~Hz}, 2 \mathrm{H}), 7.43(\mathrm{t}, J=7.6$ $\mathrm{Hz}, 2 \mathrm{H}), 7.25(\mathrm{~d}, J=8.0 \mathrm{~Hz}, 2 \mathrm{H}), 6.92(\mathrm{~d}, J=8 \mathrm{~Hz}, 2 \mathrm{H}), 3.10(\mathrm{~s}, 3 \mathrm{H}) ;{ }^{13} \mathrm{C} \mathrm{NMR}\left(100 \mathrm{MHz}, \mathrm{CDCl}_{3}\right): \delta 150.14$, $144.88,133.32,131.70,130.21,129.07,128.59,128.41,125.93,125.49,124.57,117.98,112.19,41.24 ; \mathrm{HR}$ MS (m/z M ): 322.1467, calculated for $\mathrm{C}_{23} \mathrm{H}_{18} \mathrm{~N}_{2}: 322.1470$.

10-[4-(2,5-Di-thiophen-2-yl-pyrrol-1-yl)-phenyl]-anthracene-9-carbonitrile (DTP-PAN): Yellow solid, mp: $238{ }^{\circ} \mathrm{C} ;{ }^{1} \mathrm{H}$ NMR $\left(400 \mathrm{MHz}, \mathrm{CDCl}_{3}\right): \delta 8.51(\mathrm{~d}, J=8.4 \mathrm{~Hz}, 2 \mathrm{H}), 7.73(\mathrm{~m}, 4 \mathrm{H}), 7.56(\mathrm{~d}, J=7.6 \mathrm{~Hz}, 2 \mathrm{H})$, $7.50(\mathrm{t}, J=7.6 \mathrm{~Hz}, 2 \mathrm{H}), 7.43(\mathrm{~d}, J=7.6 \mathrm{~Hz}, 2 \mathrm{H}), 7.18(\mathrm{~d}, J=6.0 \mathrm{~Hz}, 2 \mathrm{H}), 6.95(\mathrm{t}, J=4.4 \mathrm{~Hz}, 2 \mathrm{H}), 6.81(\mathrm{~d}, J$ $=4.0 \mathrm{~Hz}, 2 \mathrm{H}), 6.61(\mathrm{~s}, 2 \mathrm{H}) ;{ }^{13} \mathrm{C} \mathrm{NMR}\left(100 \mathrm{MHz}, \mathrm{CDCl}_{3}\right): \delta 141.77,138.07,137.50,134.38,132.56,131.12$, $130.03,129.82,128.91,128.29,126.95,126.51,126.17,125.22,124.27,124.07,116.99,109.90,105.89 ;$ HR MS (m/z M $\left.{ }^{+}\right): 508.1068$, calculated for $\mathrm{C}_{33} \mathrm{H}_{20} \mathrm{~N}_{2} \mathrm{~S}_{2}: 508.6065$.

10-\{4-[Bis-(4-methoxy-phenyl)-amino]-phenyl $\}$-anthracene-9-carbonitrile $\left(\mathrm{An}_{2} \mathrm{~N}\right.$-PAN): Red solid, mp: $168{ }^{\circ} \mathrm{C} ;{ }^{1} \mathrm{H}$ NMR $\left(400 \mathrm{MHz}, \mathrm{CDCl}_{3}\right): \delta 8.45(\mathrm{~d}, J=8.4 \mathrm{~Hz}, 2 \mathrm{H}), 7.89(\mathrm{~d}, J=8.4 \mathrm{~Hz}, 2 \mathrm{H}), 7.68(\mathrm{t}, J=7.6$ $\mathrm{Hz}, 2 \mathrm{H}), 7.47(\mathrm{~d}, J=7.6 \mathrm{~Hz}, 2 \mathrm{H}), 7.21(\mathrm{~d}, J=8.0 \mathrm{~Hz}, 2 \mathrm{H}), 7.15(\mathrm{~d}, J=8.4 \mathrm{~Hz}, 2 \mathrm{H}), 7.08(\mathrm{~d}, J=8.4 \mathrm{~Hz}, 2 \mathrm{H})$, $6.89(\mathrm{~d}, J=8.0 \mathrm{~Hz}, 4 \mathrm{H}), 3.81(\mathrm{~s}, 6 \mathrm{H}) ;{ }^{13} \mathrm{C} \mathrm{NMR}\left(100 \mathrm{MHz}, \mathrm{CDCl}_{3}\right): \delta 155.69,148.25,143.84,139.99,132.78$, $130.95,129.52,128.17,127.75,127.62,126.82,125.60,125.06,118.67,117.39,114.58,104.80,55.66 ;$ HR MS (m/z M $\left.{ }^{+}\right): 506.1992$, calculated for $\mathrm{C}_{35} \mathrm{H}_{26} \mathrm{~N}_{2} \mathrm{O}_{2}: 506.1994$.

General procedure for the synthesis of internal alkynes. ${ }^{7}$ 9-Bromo-10-cyanoanthracene, palladium catalyst (2 mol \%), CuI (2 mol \%), triphenylphosphine (10 mol \%) and a magnetic stirring bar were placed in a two-neck round bottom flask fitted with a condenser. The whole set up was degassed and back-filled with gaseous mixture of argon and hydrogen. To the reaction flask was added previously degassed $5 \mathrm{~mL}$ THF and TEA (6 mmol) using syringes. The terminal acetylene was dissolved in $5 \mathrm{~mL}$ THF and added to the reaction mixture at about $80{ }^{\circ} \mathrm{C}$. The reaction mixture was stirred at reflux for $24 \mathrm{hrs}$ under the atmosphere of the gas 
mixture. The solvents were evaporated and the crude product was extracted with ether $(25 \mathrm{~mL}+2 \times 15 \mathrm{~mL})$.

The combined organic layers were washed with water followed by brine before drying and evaporating. The residue after evaporation was chromatographed on silica gel using ethyl acetate - hexane mixture (1:9) to separate the product and unreacted starting compounds. Following are the characterization data: 10-

Phenylethynyl-anthracene-9-carbonitrile (PEAN): ${ }^{7}$ Pale yellow solid; mp: 208-211 ${ }^{\circ} \mathrm{C} ;{ }^{1} \mathrm{H}-\mathrm{NMR}$ : $\delta$ ppm $8.65(\mathrm{~d}, J=8.8 \mathrm{~Hz}, 2 \mathrm{H}), 8.39(\mathrm{~d}, J=8.8 \mathrm{~Hz}, 2 \mathrm{H}), 7.76(\mathrm{~m}, 2 \mathrm{H}), 7.71(\mathrm{t}, J=7.2 \mathrm{~Hz}, 2 \mathrm{H}), 7.65(\mathrm{t}, J=7.2 \mathrm{~Hz}$, 2H), 7.47 (m, 3H); ${ }^{13} \mathrm{C}-\mathrm{NMR}: \delta$ 132.20, 131.46, 130.99, 129.02, 128.60, 128.29, 127.14, 126.90, 125.37, 123.69, 122.23, 116.91, 105.59, 104.62, 85.26; HR MS (m/z M $\left.{ }^{+}\right): 303.1047$ Calculated for $\mathrm{C}_{23} \mathrm{H}_{13} \mathrm{~N}$ : 303.1048 .

10-(4-Methoxy-phenylethynyl)-anthracene-9-carbonitrile (AnEAN): ${ }^{21 \mathrm{e}}$ Bright yellow solid; mp: 190$192{ }^{\circ} \mathrm{C} ;{ }^{1} \mathrm{H}-\mathrm{NMR}(400 \mathrm{MHz}):, \delta 8.65(\mathrm{~d}, J=8.4 \mathrm{~Hz}, 2 \mathrm{H}), 8.39(\mathrm{~d}, J=8.4 \mathrm{~Hz}, 2 \mathrm{H}), 7.70(\mathrm{~m}, 4 \mathrm{H}), 7.64(\mathrm{t}, J=$ $8.4 \mathrm{~Hz}, 2 \mathrm{H}), 6.98(\mathrm{~d}, J=8.8 \mathrm{~Hz}, 2 \mathrm{H}), 3.89(\mathrm{~s}, 3 \mathrm{H}) ;{ }^{13} \mathrm{C}-\mathrm{NMR}: \delta=159.91,133.04,132.21,130.75,128.52$, $127.20,126.66,125.31,124.21,117.01,114.35,114.04,105.14,104.96,84.40,55.58 ; \mathrm{HR} \mathrm{MS}\left(\mathrm{m} / \mathrm{z} \mathrm{M}^{+}\right)$: 333.1154 Calculated for $\mathrm{C}_{24} \mathrm{H}_{15} \mathrm{NO}: 333.1154$.

10-(4-Dimethylamino-phenylethynyl)-anthracene-9-carbonitrile (DMA-PEAN): ${ }^{21 \mathrm{e}}$ Red needles; mp: 200-202 ${ }^{\circ} \mathrm{C} ;{ }^{1} \mathrm{H}-\mathrm{NMR}: \delta 8.67(\mathrm{~d}, J=8.4 \mathrm{~Hz}, 2 \mathrm{H}), 8.37(\mathrm{~d}, J=8.4 \mathrm{~Hz}, 2 \mathrm{H}), 7.69(\mathrm{t}, J=7.6 \mathrm{~Hz}, 2 \mathrm{H}), 7.63(\mathrm{~m}$, 4H), $6.71(\mathrm{~d}, J=7.6 \mathrm{~Hz}, 2 \mathrm{H}), 3.05(\mathrm{~s}, 6 \mathrm{H}) ;{ }^{13} \mathrm{C}-\mathrm{NMR}: \delta 150.25,132.82,132.40,130.56,128.50,127.47$, $126.40,125.29,125.18,117.29,111.53,108.73,107.37,104.03,84.36,40.43 ; \mathrm{HR}$ MS (m/z M+): 346.1478 Calculated for $\mathrm{C}_{25} \mathrm{H}_{18} \mathrm{~N}_{2}: 346.1470$.

\section{0-[4-(2,5-Di-thiophen-2-yl-pyrrol-1-yl)-phenylethynyl]-anthracene-9-carbonitrile}

(DTP-PEAN):

Orange needles, mp: $284{ }^{\circ} \mathrm{C} ;{ }^{1} \mathrm{H}$ NMR $\left(400 \mathrm{MHz}, \mathrm{CDCl}_{3}\right): \delta 8.64(\mathrm{~d}, J=8.4 \mathrm{~Hz}, 2 \mathrm{H}), 8.41(\mathrm{~d}, J=8.8 \mathrm{~Hz}$, 2H), $7.79(\mathrm{~d}, J=8.0 \mathrm{~Hz}, 2 \mathrm{H}), 7.73(\mathrm{t}, J=7.6 \mathrm{~Hz}, 2 \mathrm{H}), 7.66(\mathrm{t}, J=7.6 \mathrm{~Hz}, 2 \mathrm{H}), 7.38(\mathrm{~d}, J=8.0 \mathrm{~Hz}, 2 \mathrm{H}), 7.11$ $(\mathrm{d}, J=5.6 \mathrm{~Hz}, 2 \mathrm{H}), 6.86(\mathrm{t}, J=4.4 \mathrm{~Hz}, 2 \mathrm{H}), 6.61(\mathrm{~d}, J=4.0 \mathrm{~Hz}, 2 \mathrm{H}), 6.56(\mathrm{~s}, 2 \mathrm{H}) ;{ }^{13} \mathrm{C} \mathrm{NMR}(100 \mathrm{MHz}$, $\left.\mathrm{CDCl}_{3}\right): \delta 139.24,134.54,132.66,132.59,131.56,130.25,129.94,129.10,127.50,127.41,127.05,125.89$, 
$124.95,124.52,123.48,123.13,117.11,110.53,106.36,103.72,86.76 \mathrm{HR} \mathrm{MS}\left(\mathrm{m} / \mathrm{z} \mathrm{M}^{+}\right): 532.1069$ calculated for $\mathrm{C}_{35} \mathrm{H}_{20} \mathrm{~N}_{2} \mathrm{~S}_{2}: 532.1068$.

10-\{4-[Bis-(4-methoxy-phenyl)-amino]-phenylethynyl\}-anthracene-9-carbonitrile $\left(\mathrm{An}_{2} \mathrm{~N}\right.$-PEAN): Red solid, mp: $221{ }^{\circ} \mathrm{C}{ }^{1} \mathrm{H}$ NMR (400MHz, $\left.\mathrm{CDCl}_{3}\right): \delta 8.66(\mathrm{~d}, J=8.8 \mathrm{~Hz}, 2 \mathrm{H}), 8.39(\mathrm{~d}, J=8.8 \mathrm{~Hz}, 2 \mathrm{H}), 7.71(\mathrm{~d}, J$ $=7.6 \mathrm{~Hz}, 2 \mathrm{H}), 7.63(\mathrm{t}, J=7.6 \mathrm{~Hz}, 2 \mathrm{H}), 7.53(\mathrm{~d}, J=8.8 \mathrm{~Hz}, 2 \mathrm{H}), 7.12(\mathrm{~d}, J=8.4 \mathrm{~Hz}, 2 \mathrm{H}), 6.93(\mathrm{~d}, J=8.4 \mathrm{~Hz}$ 2H), $6.87(\mathrm{~d}, J=8.4 \mathrm{~Hz}, 2 \mathrm{H}), 3.82(\mathrm{~s}, 6 \mathrm{H}) ;{ }^{13} \mathrm{C} \mathrm{NMR}\left(100 \mathrm{MHz}, \mathrm{CDCl}_{3}\right): \delta 156.04,149.27,139.26,132.43$, $132.34,130.73,128.53,127.35,126.99,126.57,125.33,124.67,118.44,117.16,114.61,112.39,106.36$ 104.53, 84.65, 55.65; HR MS (m/z M ${ }^{+}$): 530.2006; calculated for $\mathrm{C}_{37} \mathrm{H}_{26} \mathrm{~N}_{2} \mathrm{O}_{2}: 530.1994$.

Theoretical calculations: Density Functional Theoretical calculations were performed using Spartan'04(W). ${ }^{8}$ Structures were drawn at the entry-level of input and minimized. Equilibrium geometry was obtain at B3LYP level of DFT for each molecule at the ground state from its initial geometry subject to symmetry with $6-31 G^{*}$ basis set. ${ }^{9}$ The total charge was kept neutral or anion/cation as required and the multiplicity at singlet or doublet as required. Orbitals and energies, atomic charges, vibrational modes and thermodynamic properties were chosen as output parameters. HOMO and LUMO orbital surfaces and electrostatic potential density maps were then obtained from the output. 


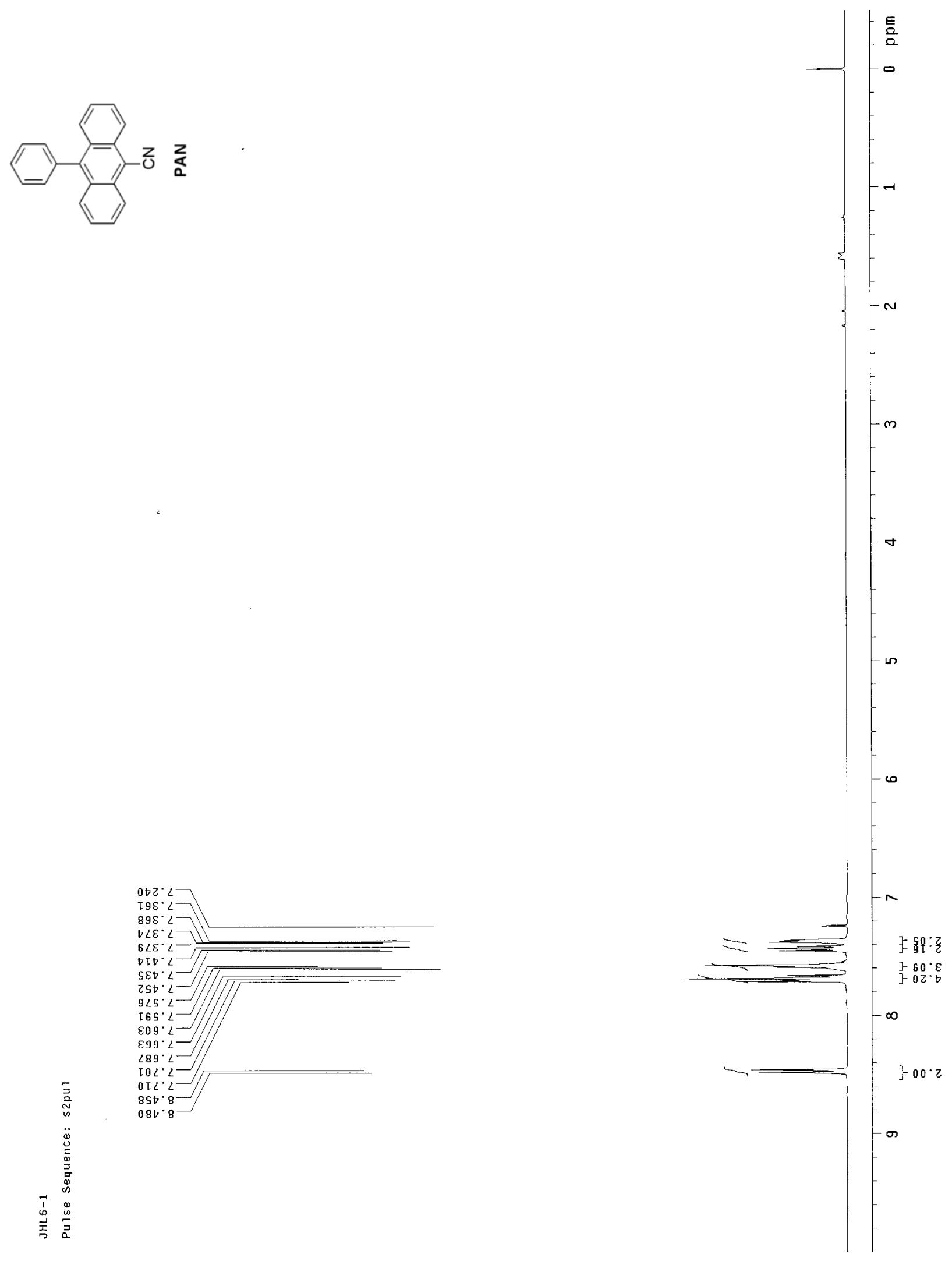



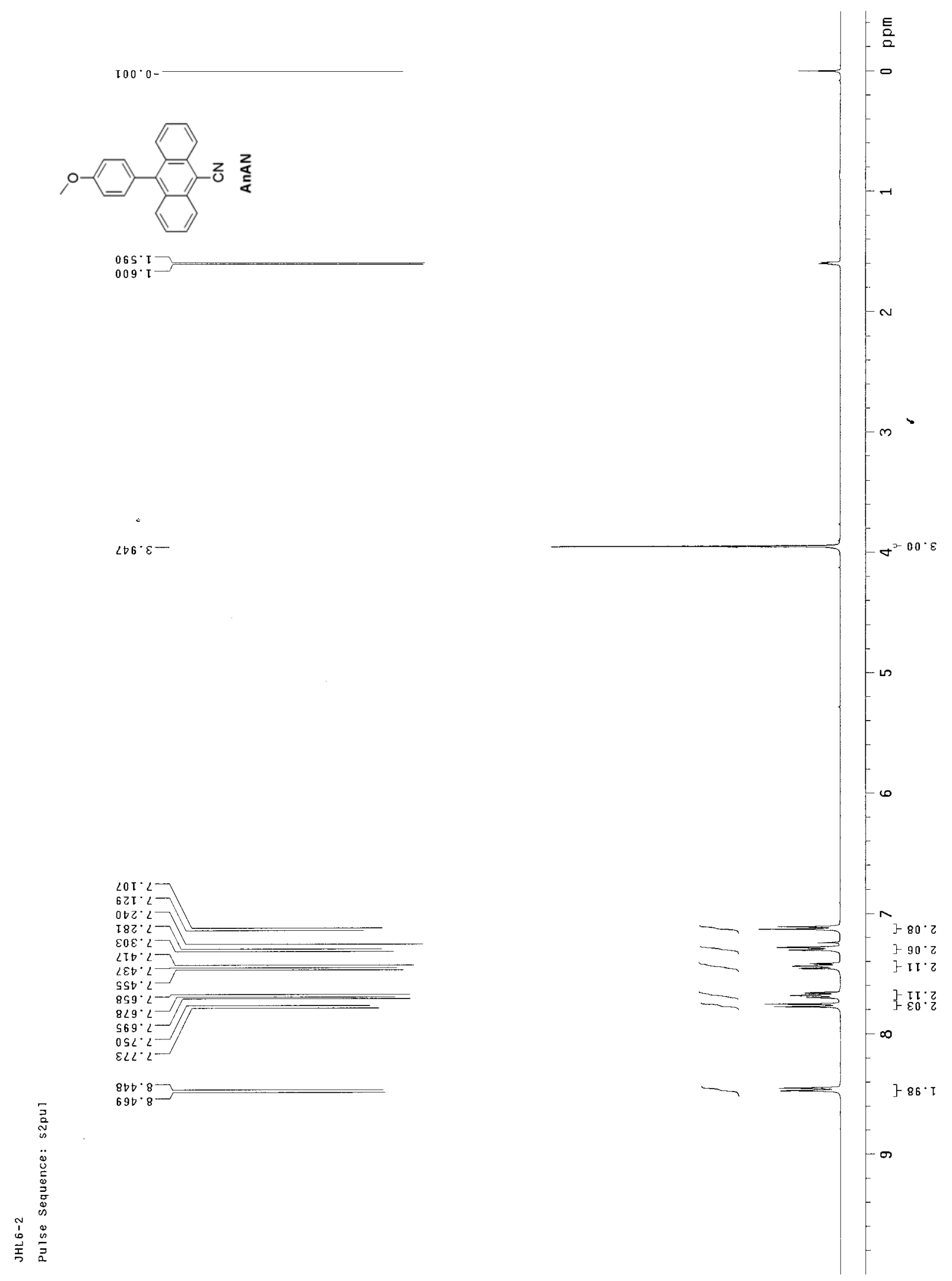


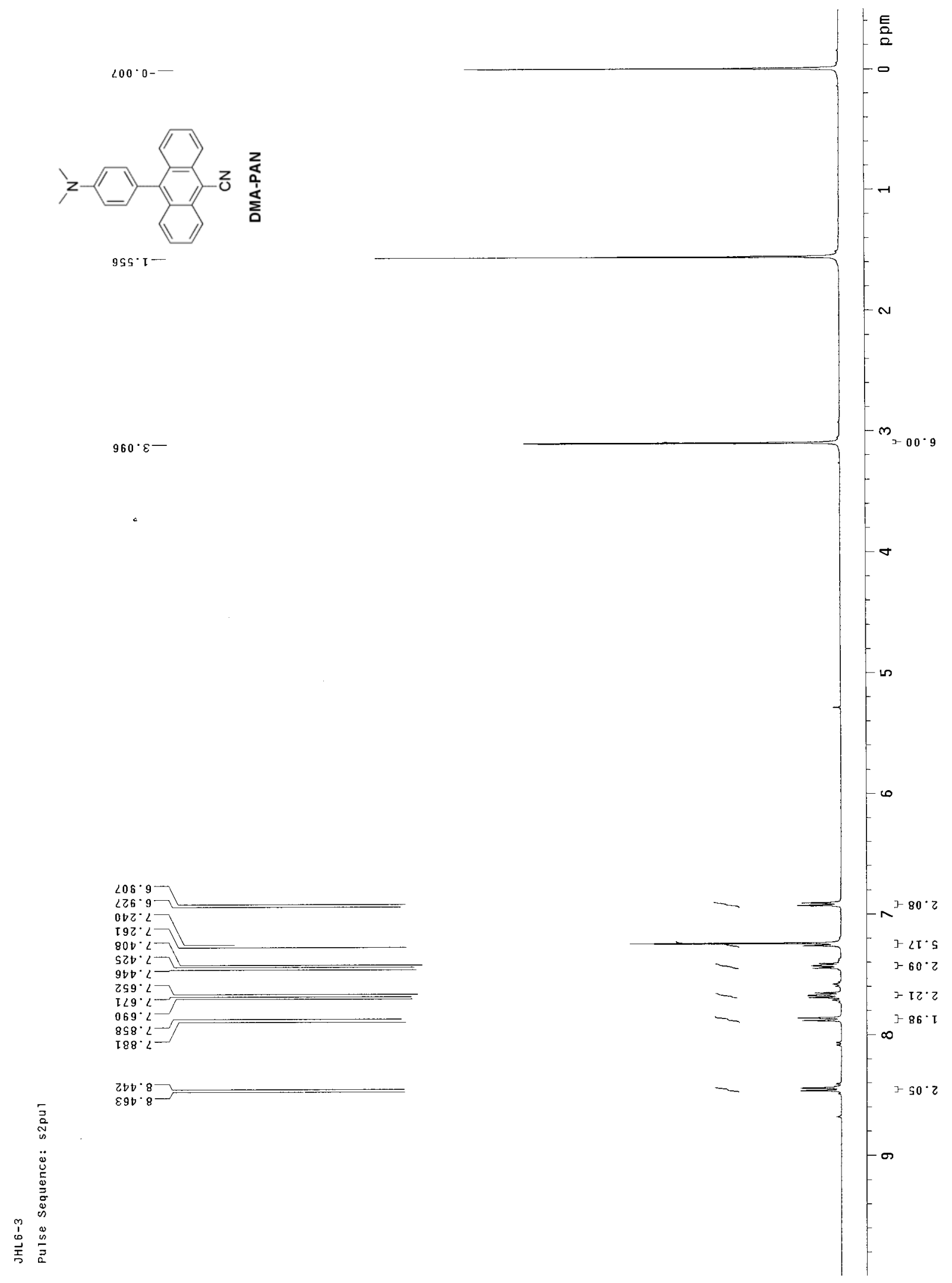



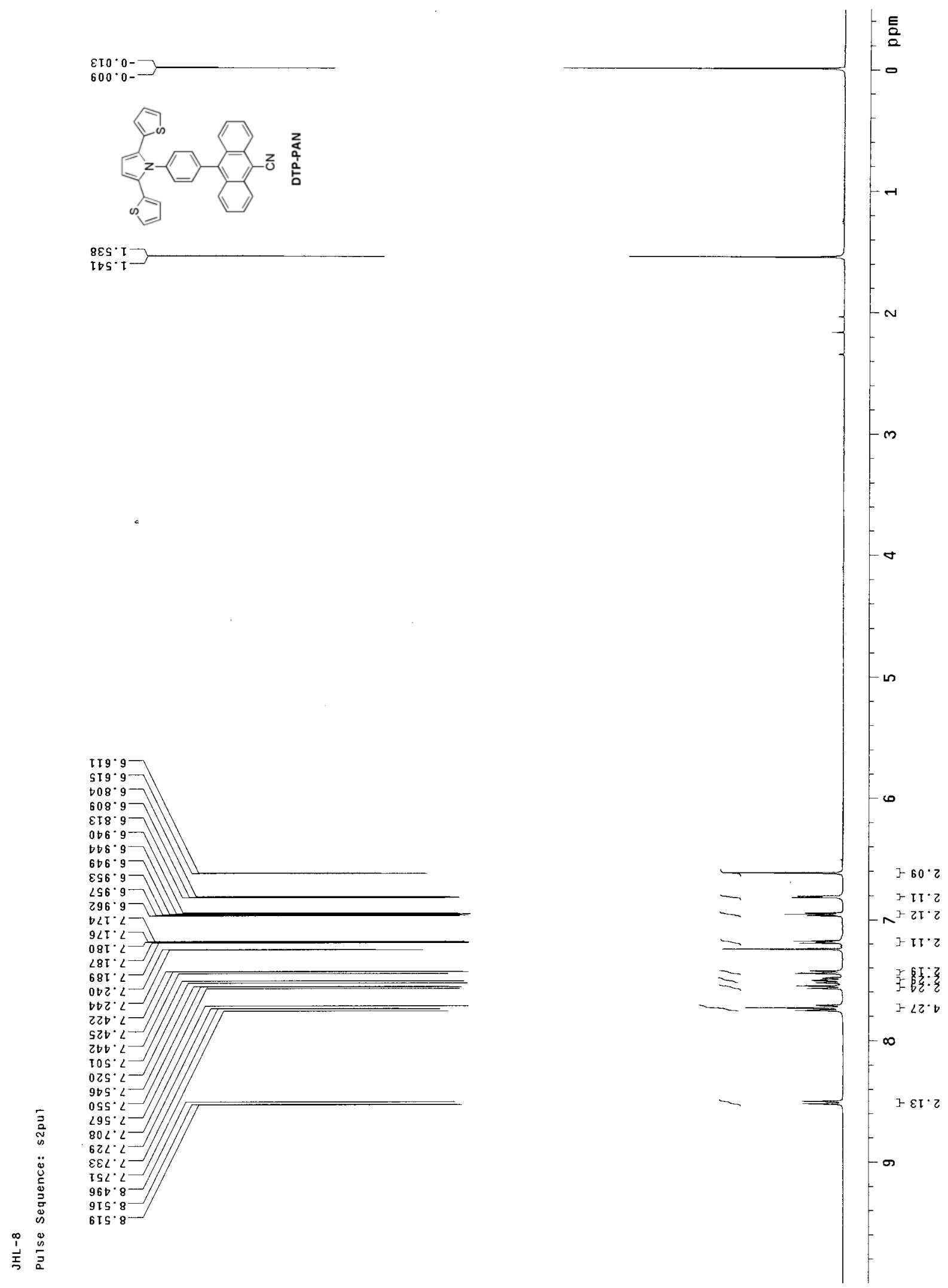


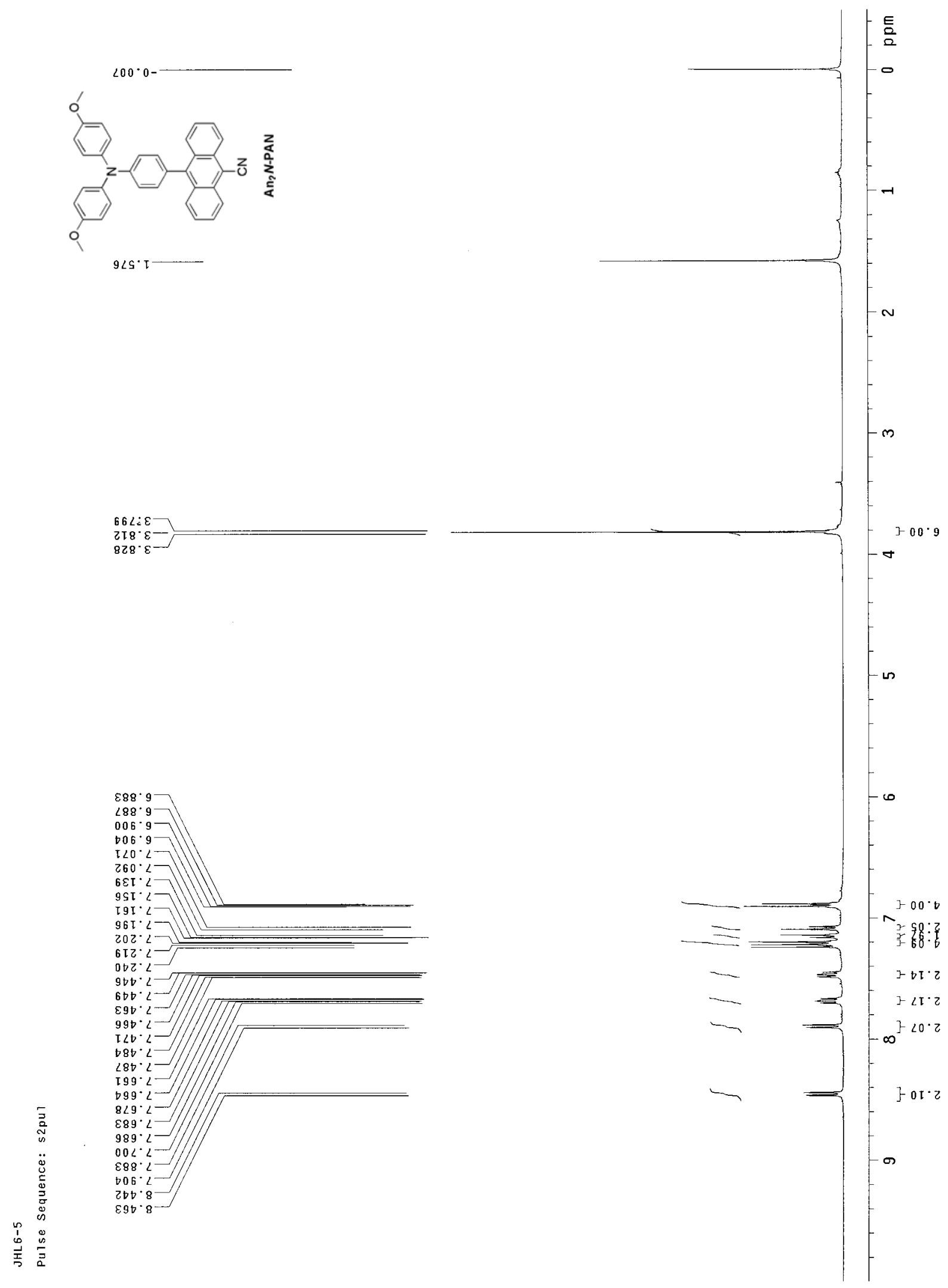



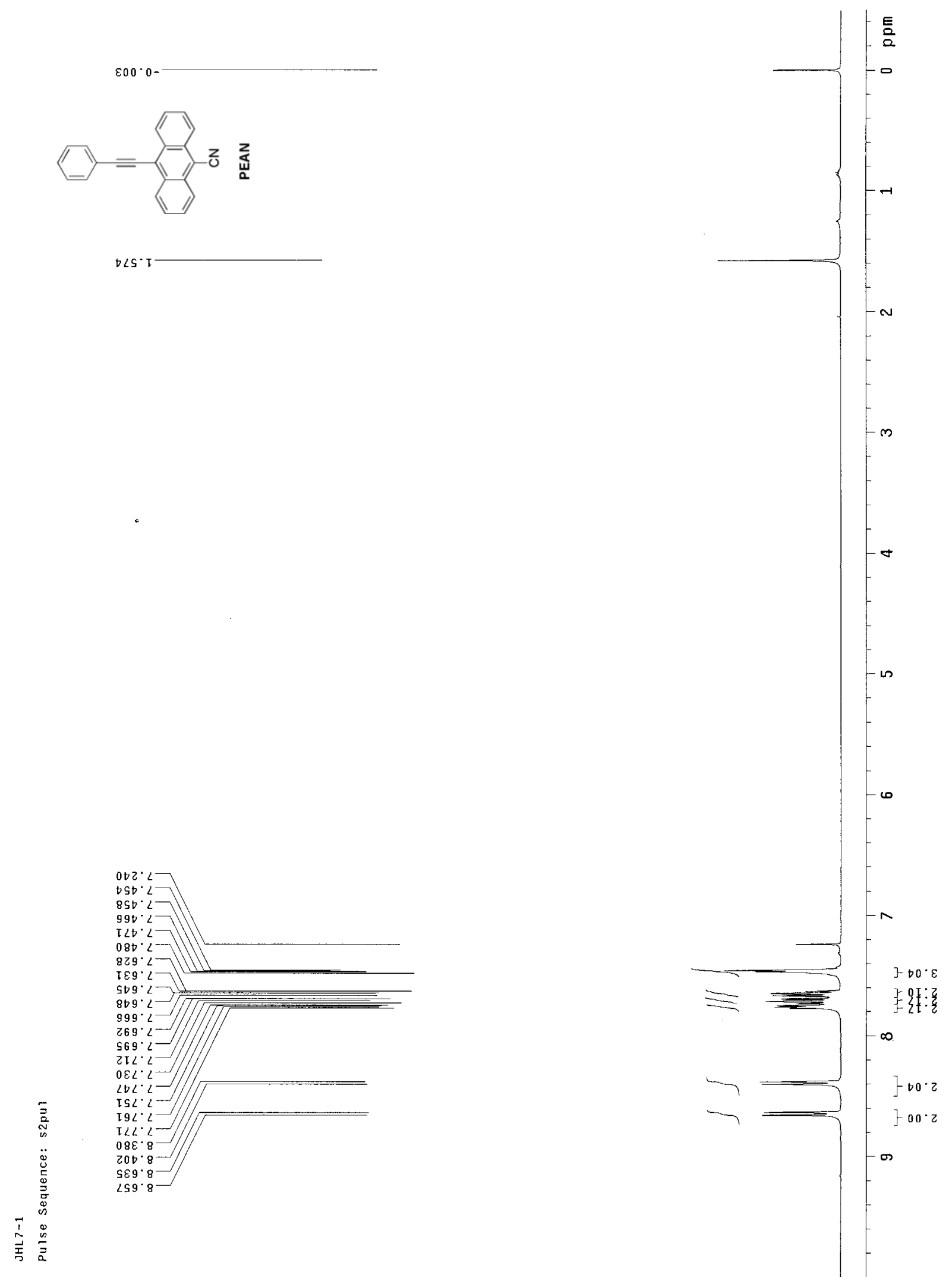


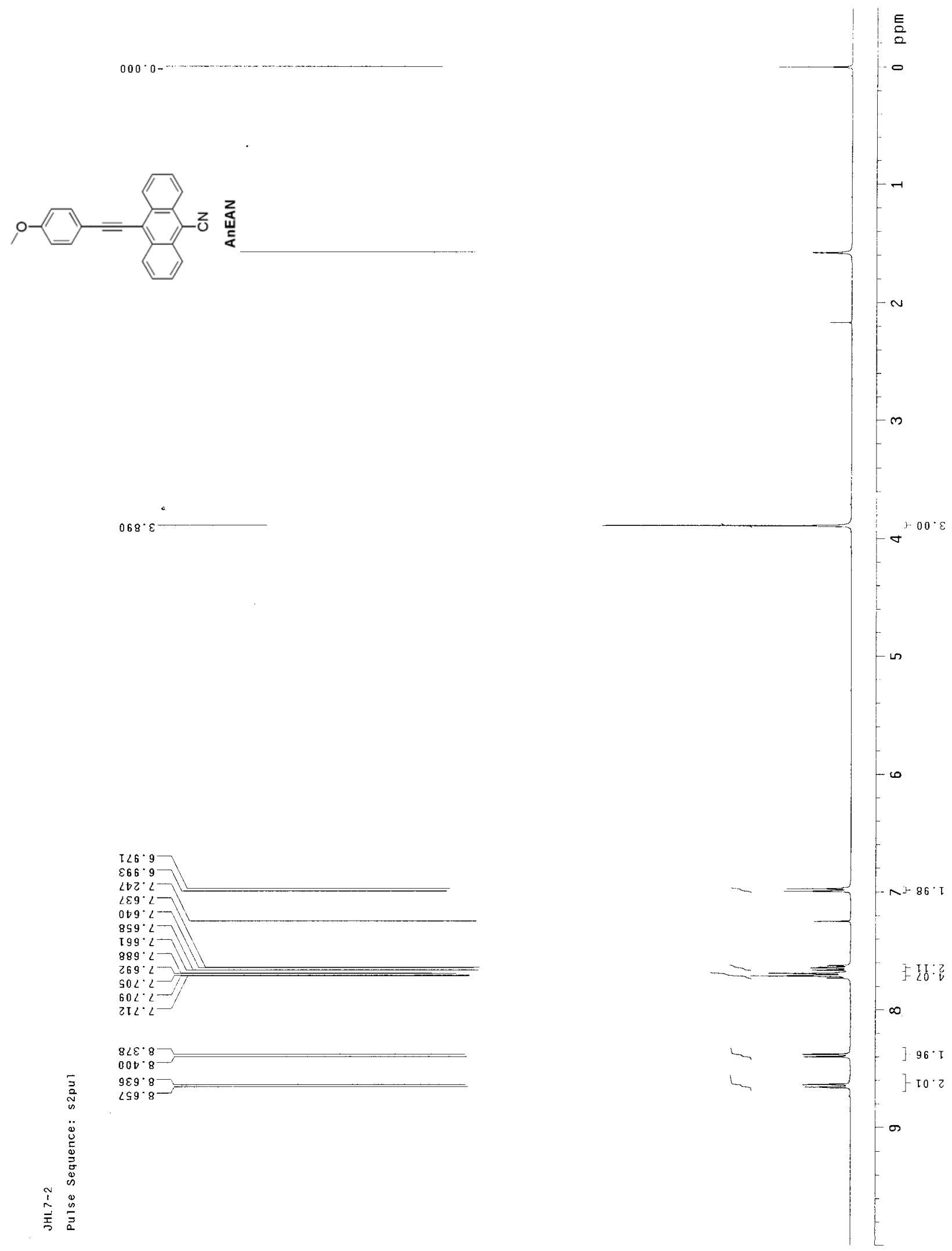




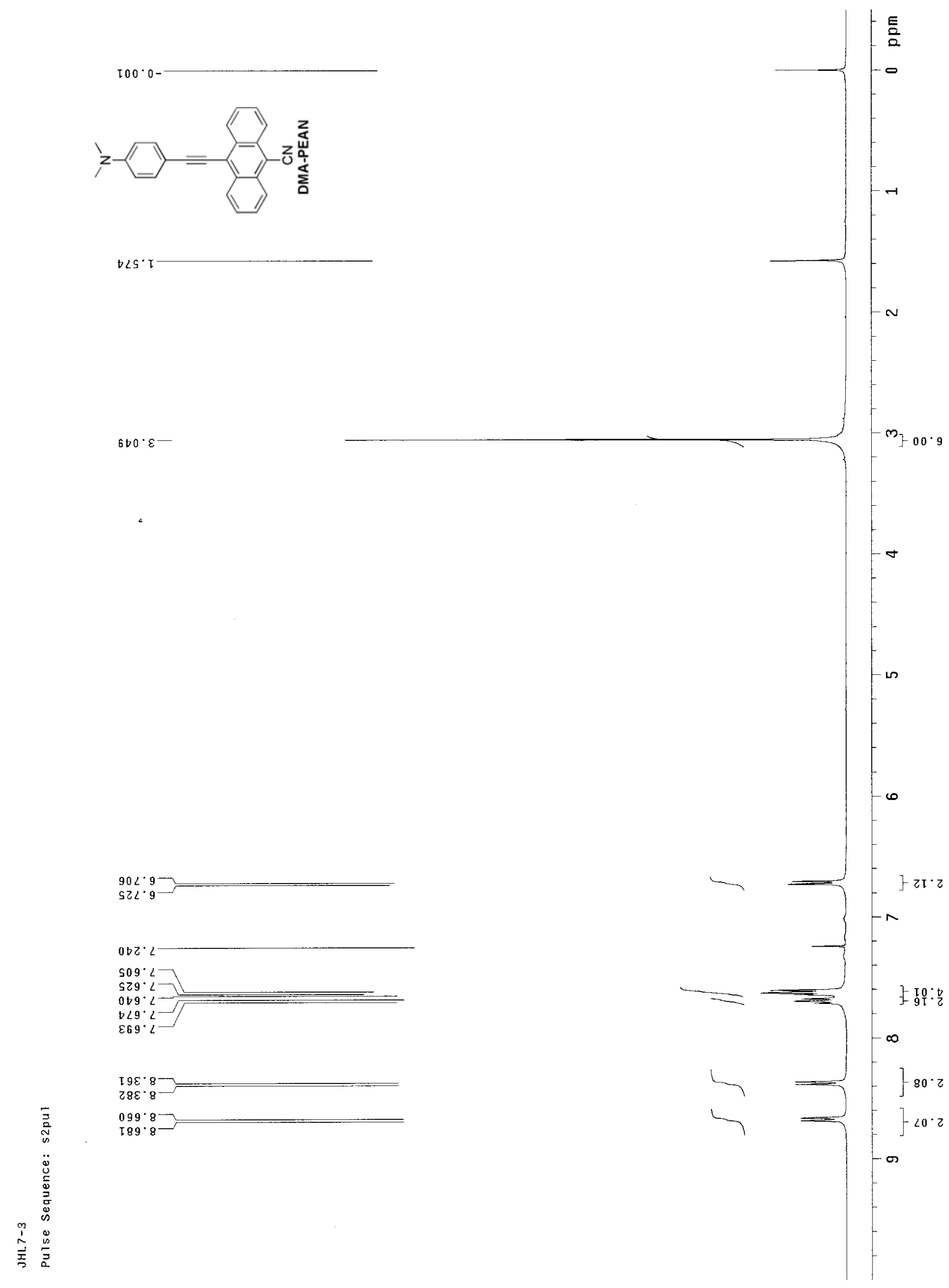




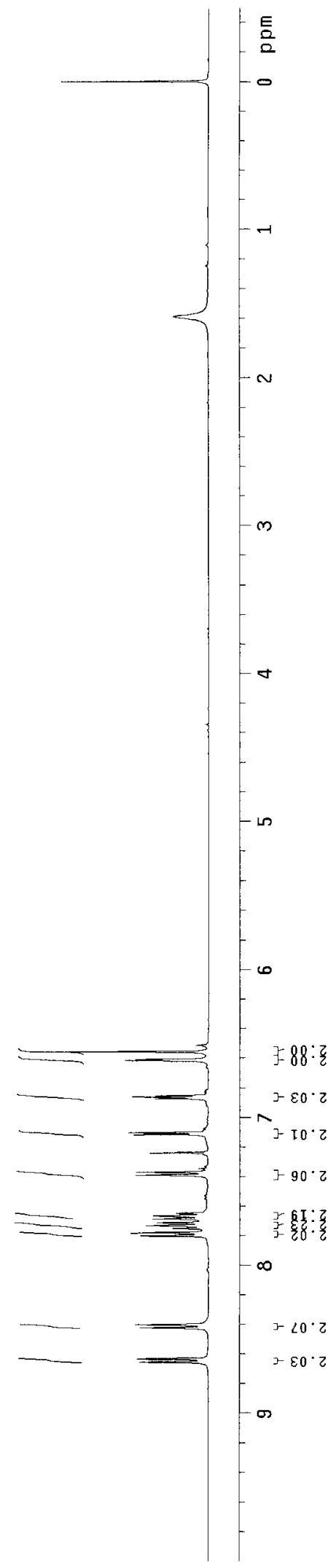

$889^{\circ} I^{2}+\ldots$

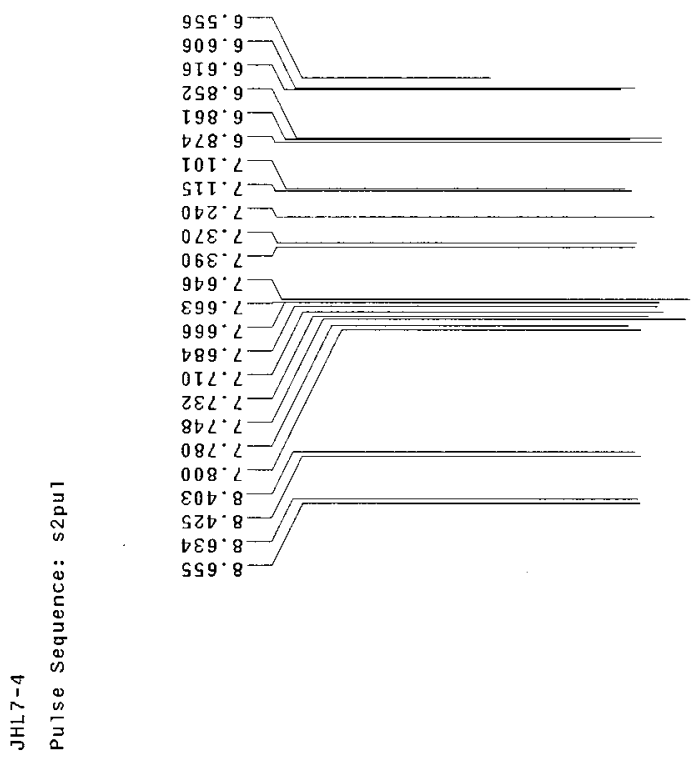




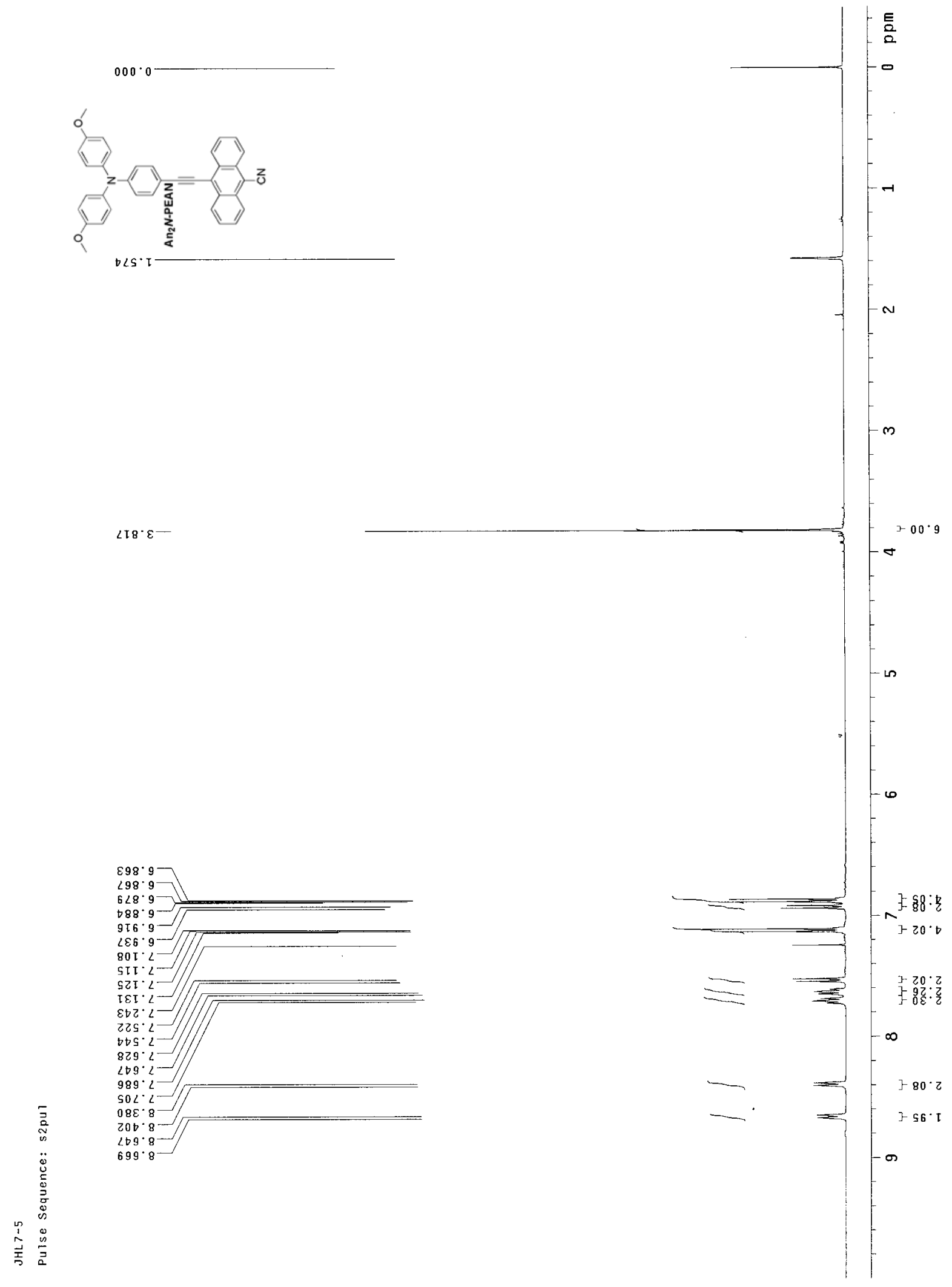



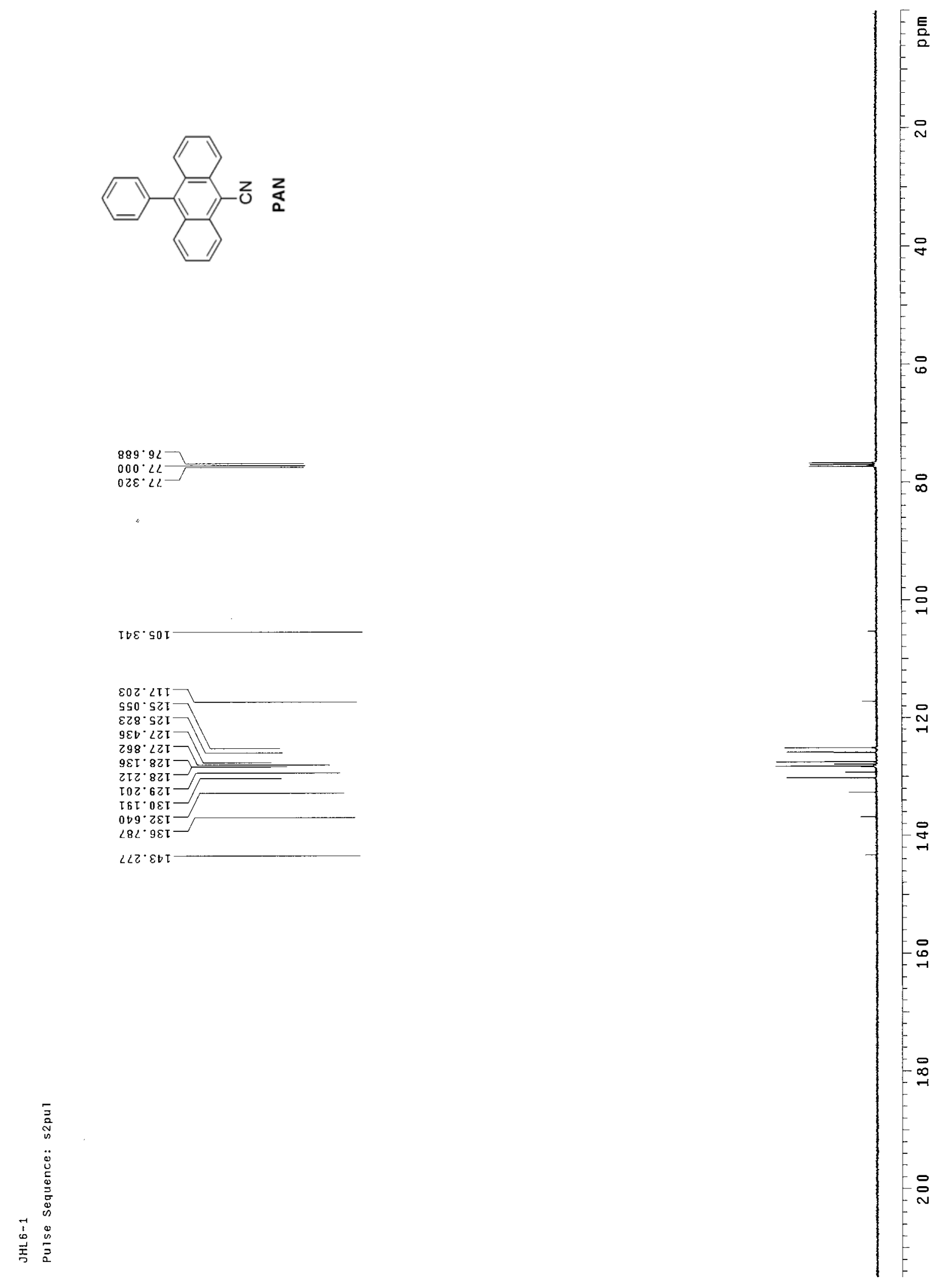

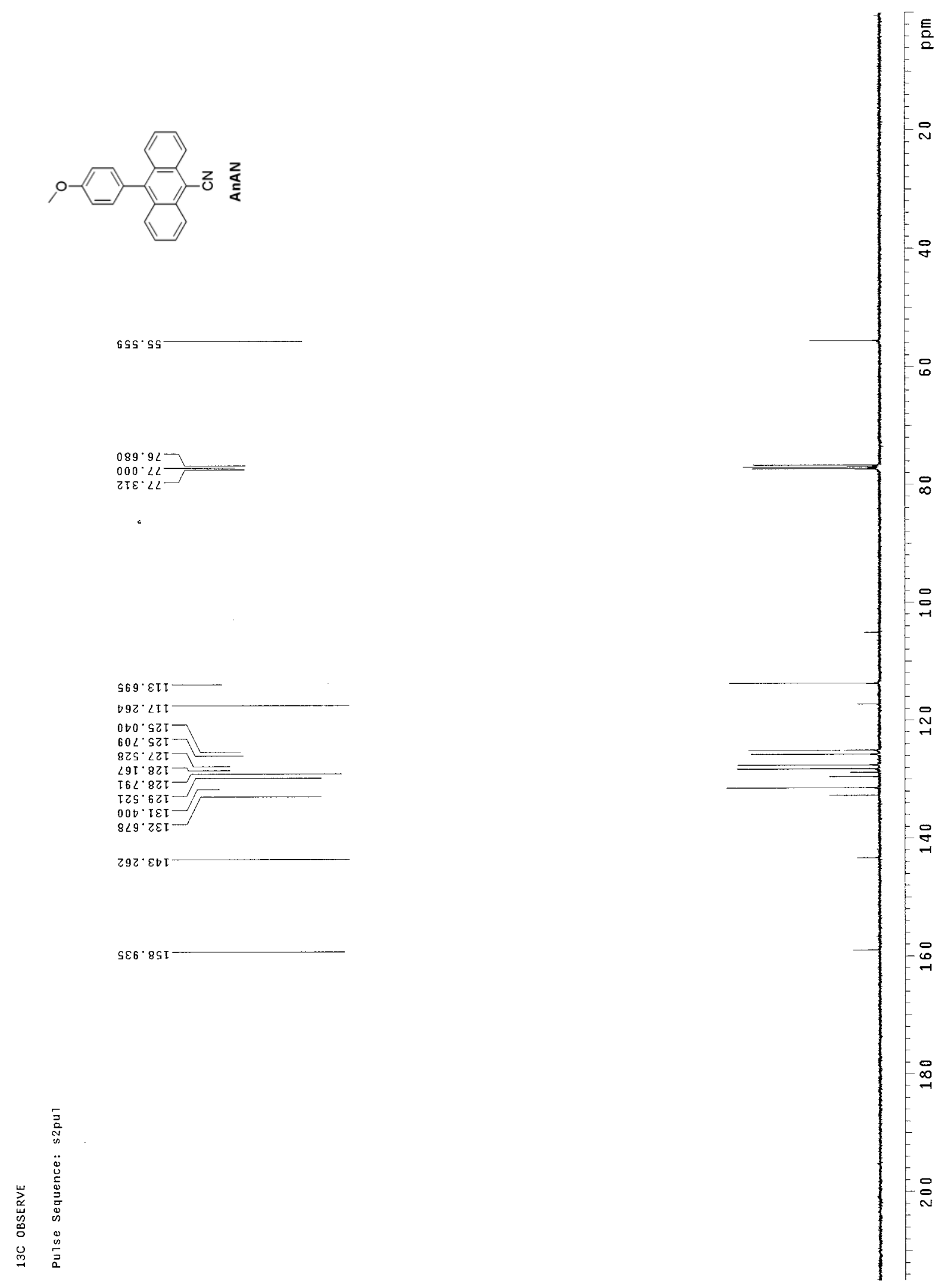


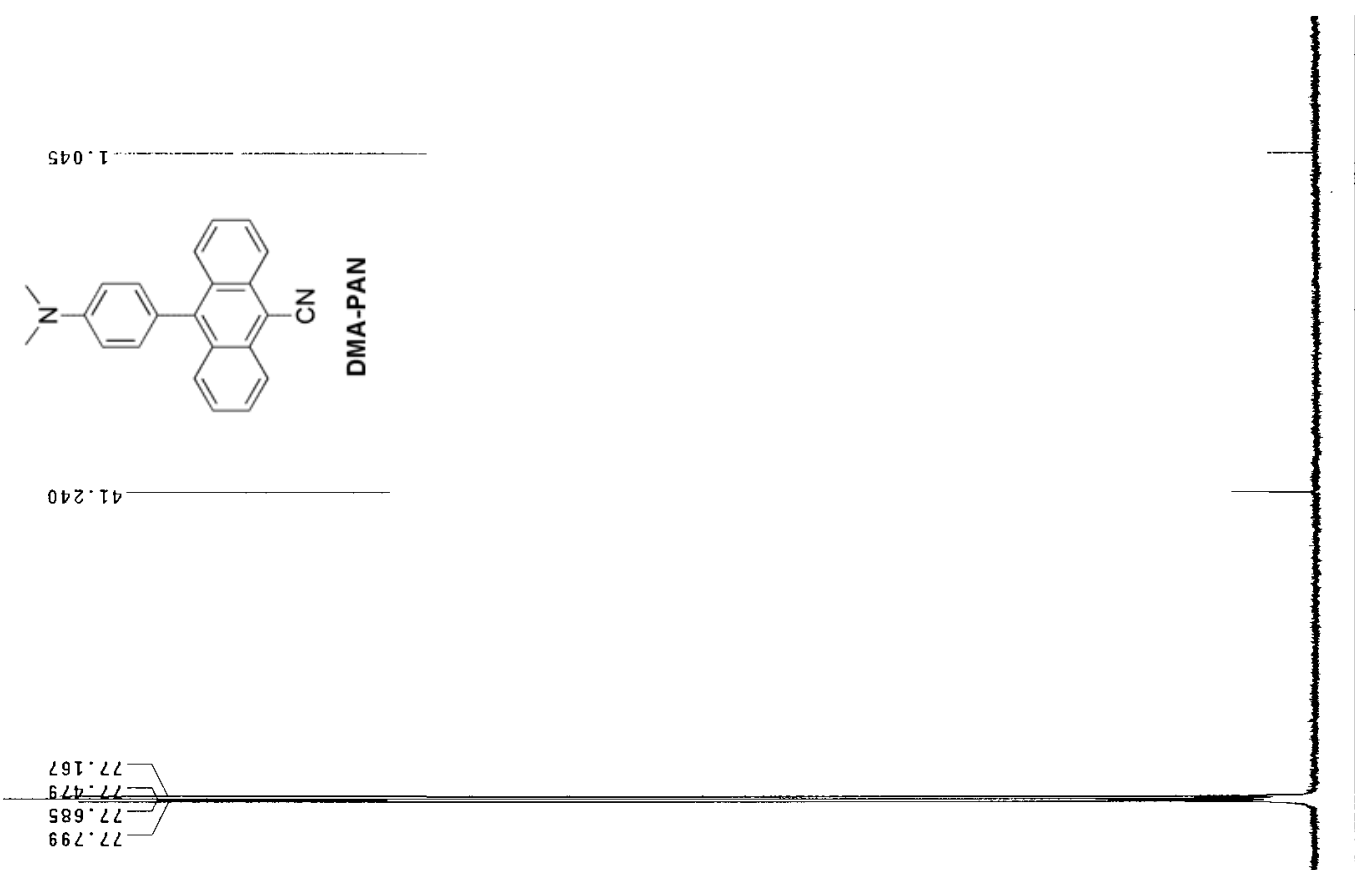

$68 \mathrm{I}^{2} \mathrm{2IT}-$

$6 \angle 6 \cdot \angle \pi T$

$895 \cdot \circ 217$

$880 \cdot 521$

$0 \varepsilon 6 \cdot s Z I$
$0 I D \cdot 8 Z T$

E6S.82I

$3 \angle 0.621$

$90 Z \cdot 0 \varepsilon \mathrm{I}$

$\angle 69^{\circ} \tau \varepsilon I$

$5 \angle 8 \cdot \circ D I$

ODI.0SI

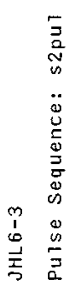



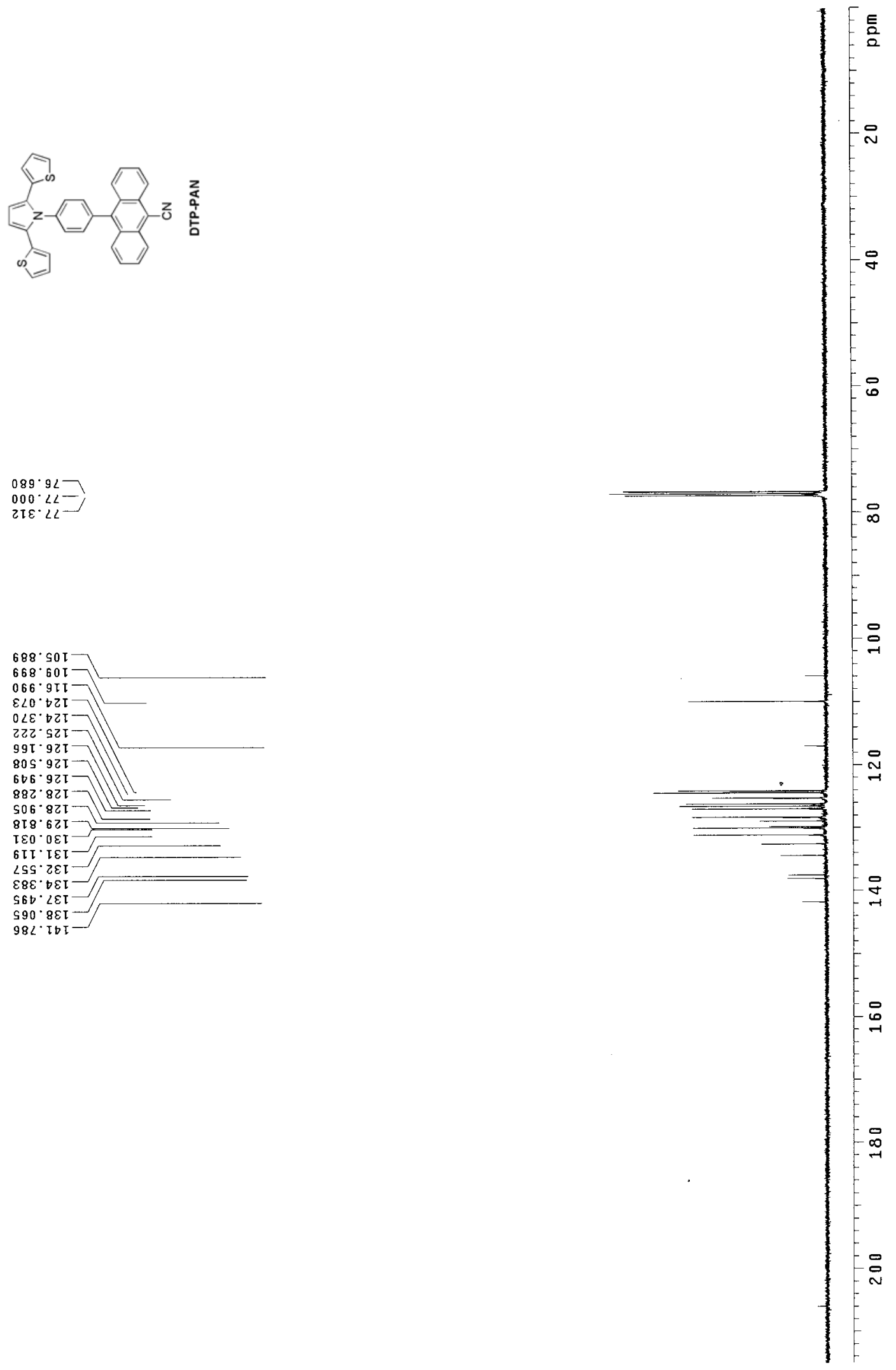

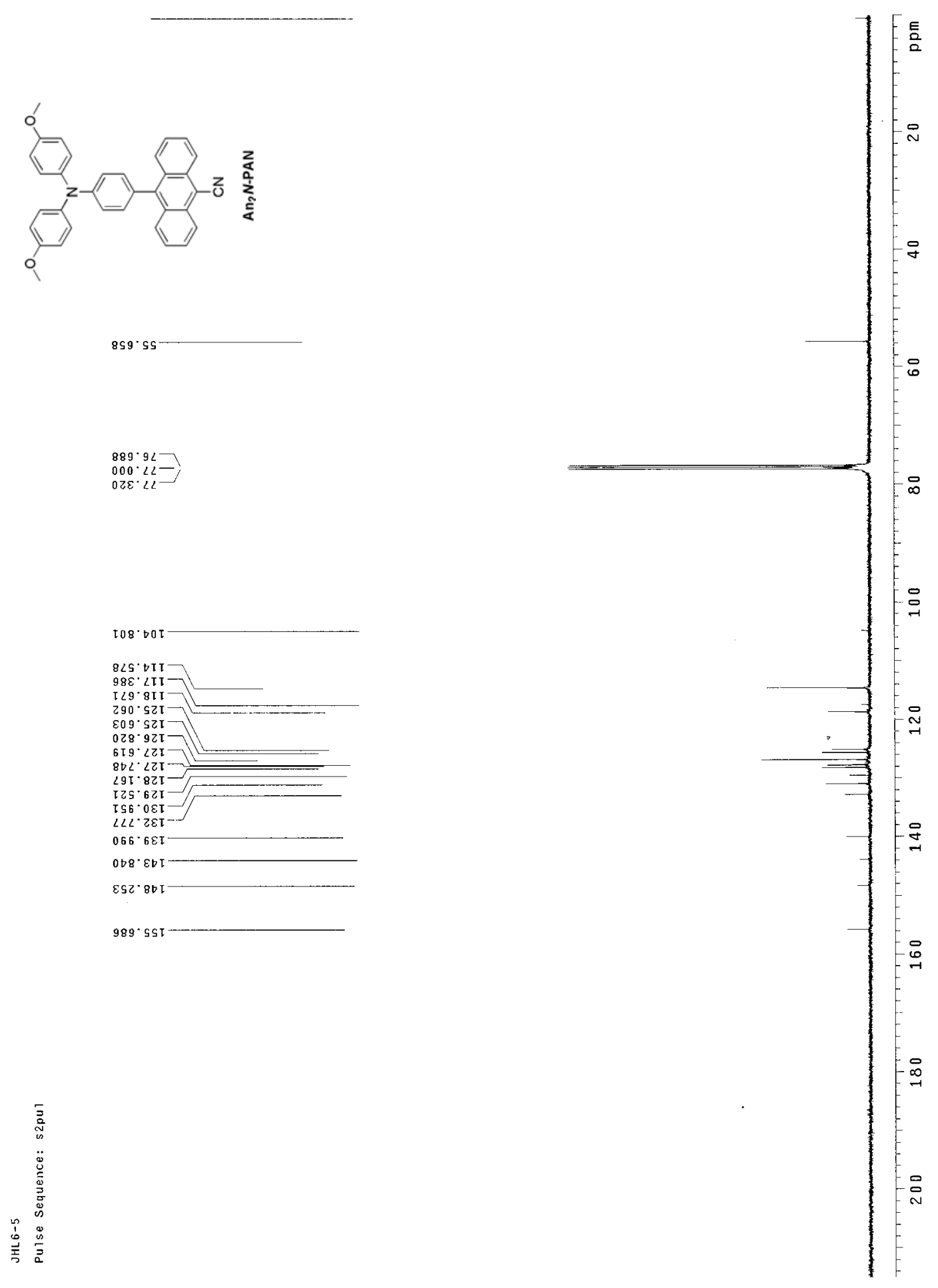

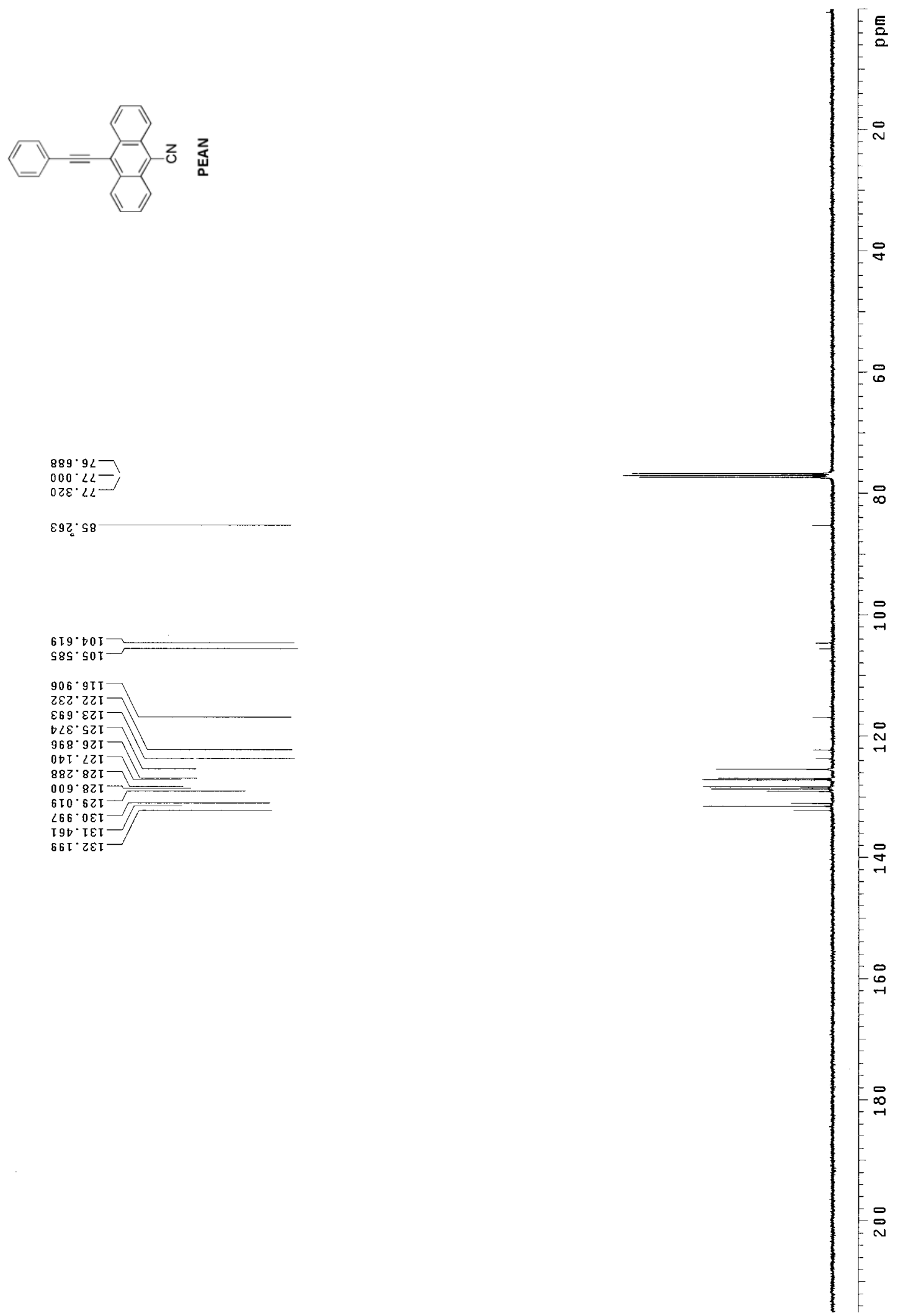

$889 \cdot 92$

$000^{\circ} \angle 2-$

892.58

S85.50T

$906 \cdot 9 I \tau$

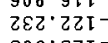

$\varepsilon 69 \cdot \varepsilon \zeta T-$

$\checkmark<\varepsilon^{\circ} s z$

968.9ZI

$0 D I \cdot \angle 2 I$

$88 \mathrm{Z}^{\circ} 8 \mathrm{I} \mathrm{I}$
$009.82 \mathrm{~T}$

$610^{\circ} 62 \mathrm{I}$

$\angle 66^{\circ} 0 E \mathrm{I}$

โ $90^{\circ}$ TEI

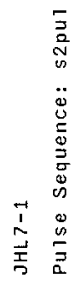



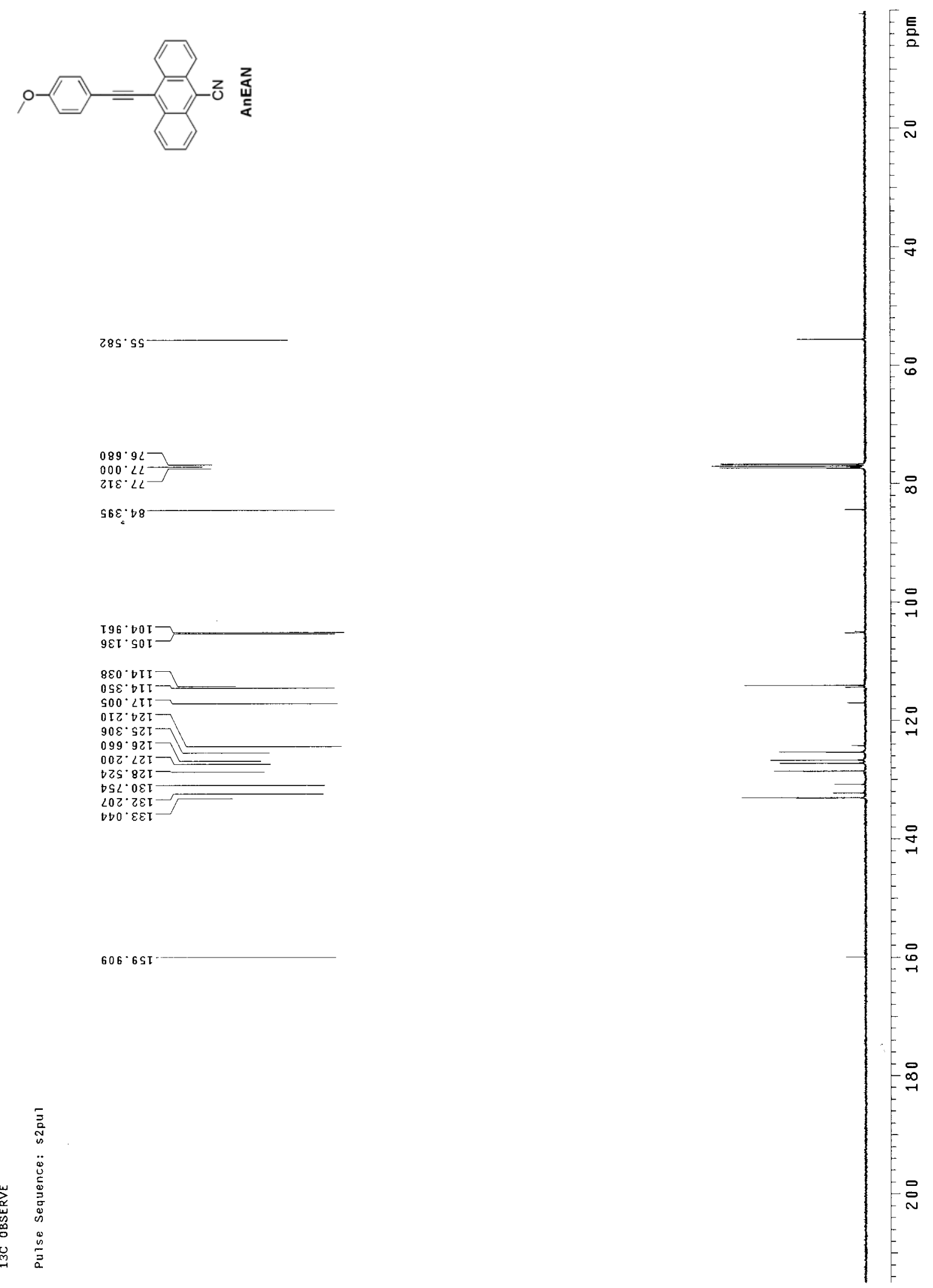

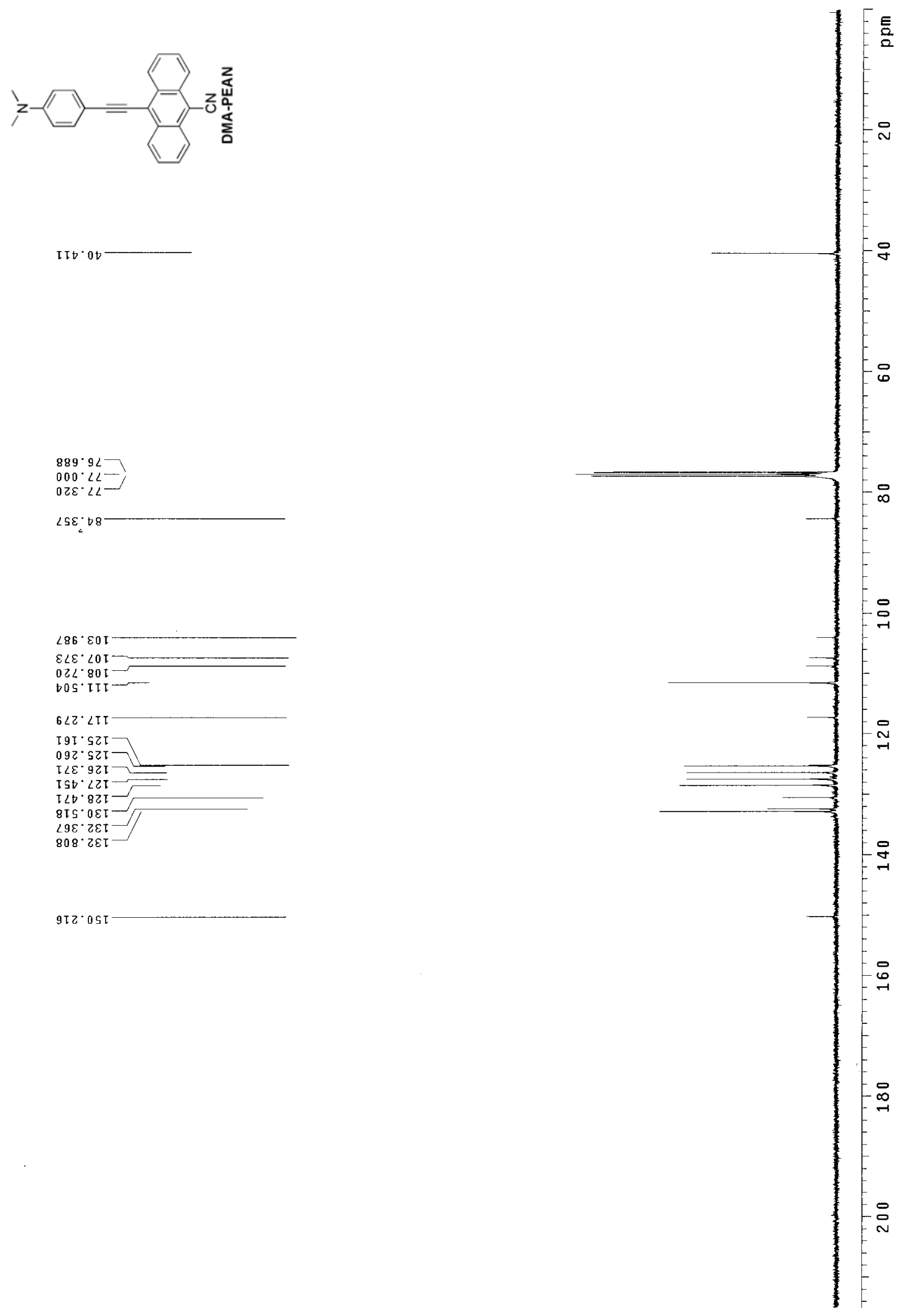


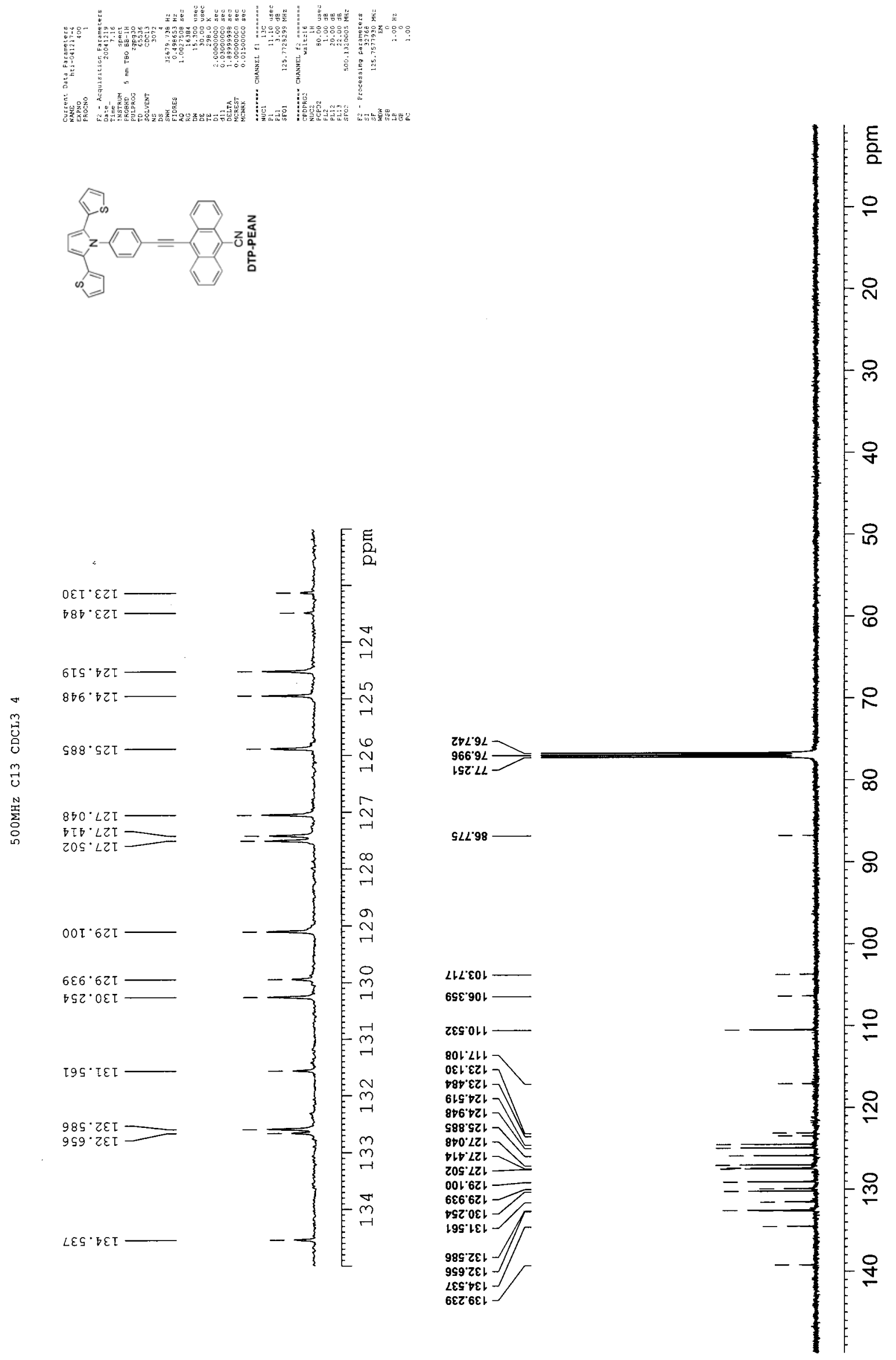



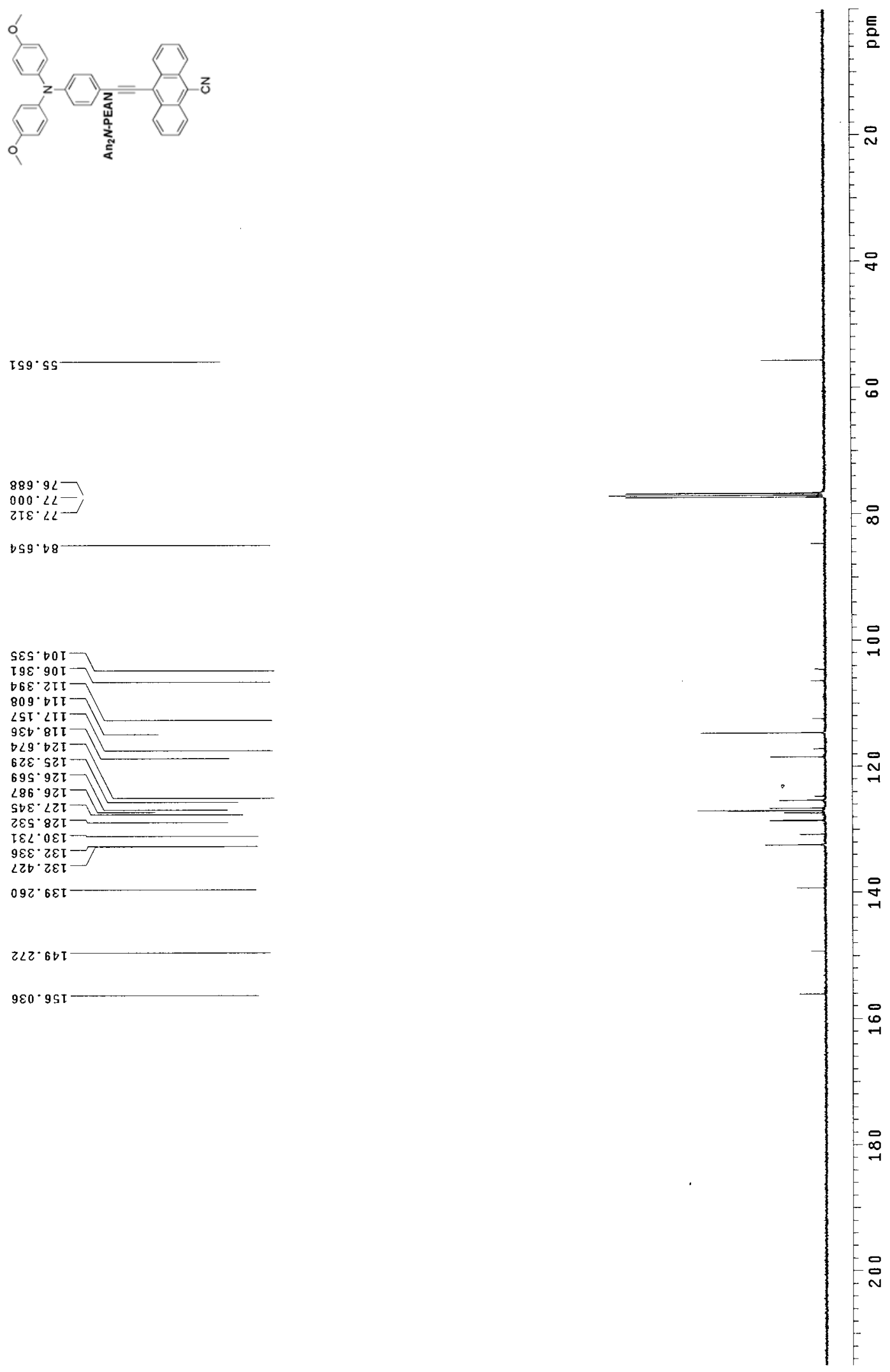
$\underline{\text { PANS }}$
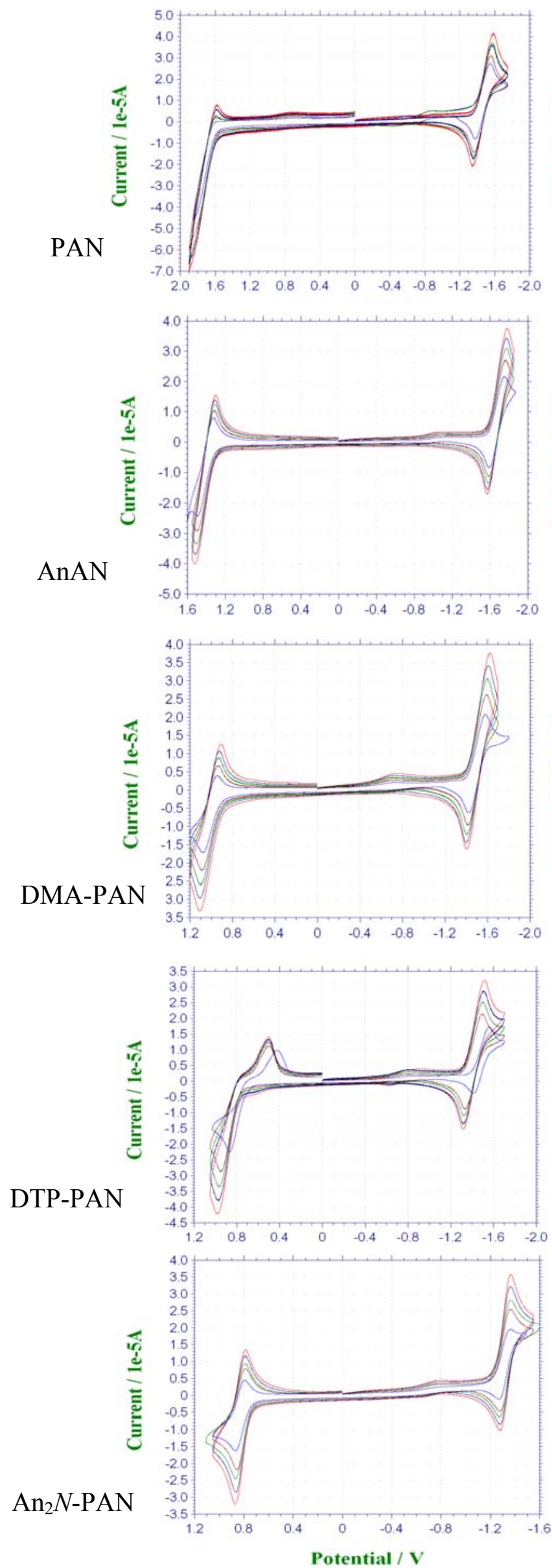

\section{PEANS}

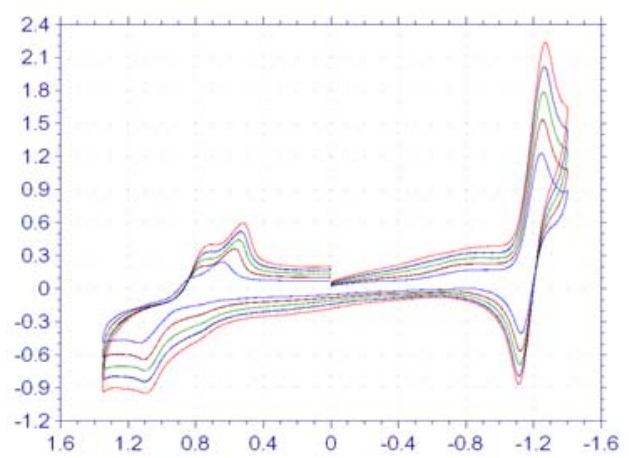

PEAN

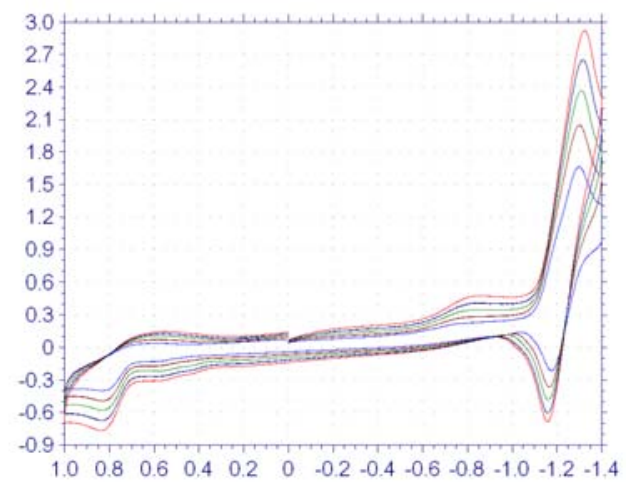

AnEAN

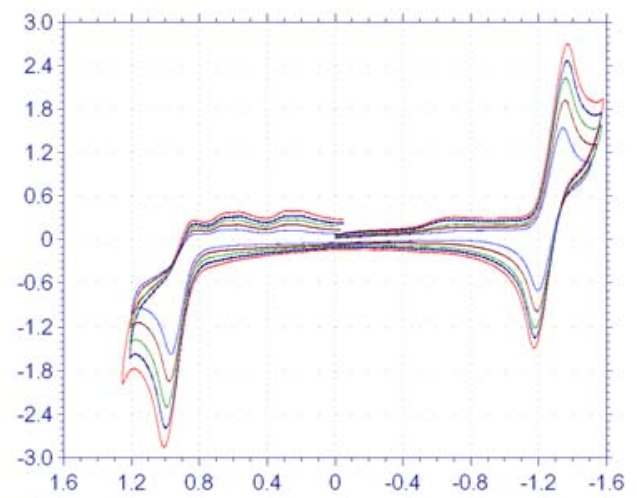

DMA-PEAN

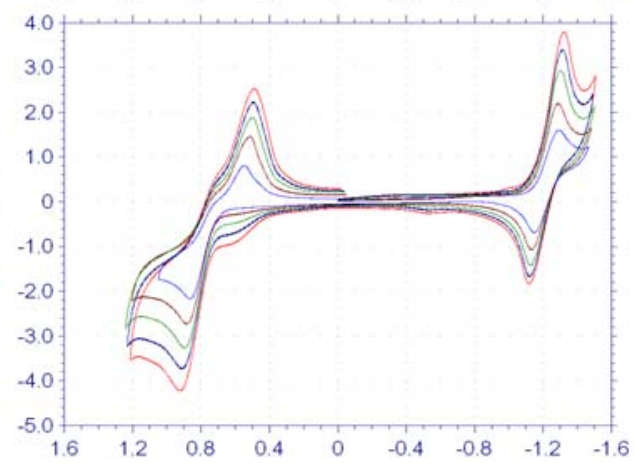

DTP-PEAN

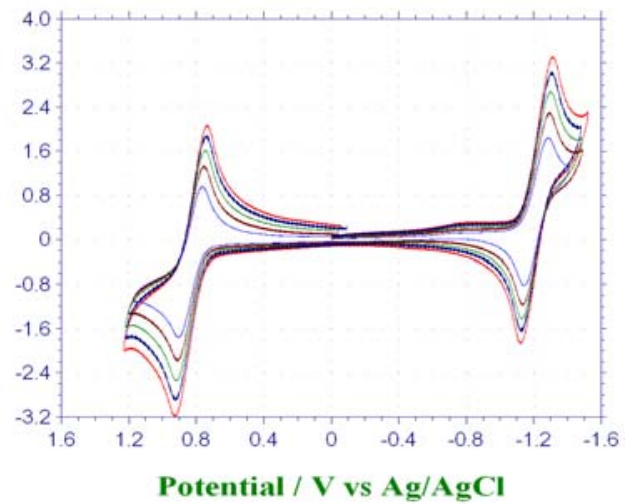

$\mathrm{An}_{2} N$-PEAN

Multi scan CV traces for PAN and PEAN series of compounds recorded reference to $\mathrm{Ag} / \mathrm{Ag}^{+}$at different scan rates in $\mathrm{CH}_{2} \mathrm{Cl}_{2}\left(10^{-3} \mathrm{M}\right.$ with $50 \mathrm{mM}$ TBAP). Color code - Red: $50 \mathrm{mV}$; blue: $75 \mathrm{mV}$; green: $100 \mathrm{mV}$; crimson: $125 \mathrm{mV}$ and navy blue: $150 \mathrm{mV}$. 

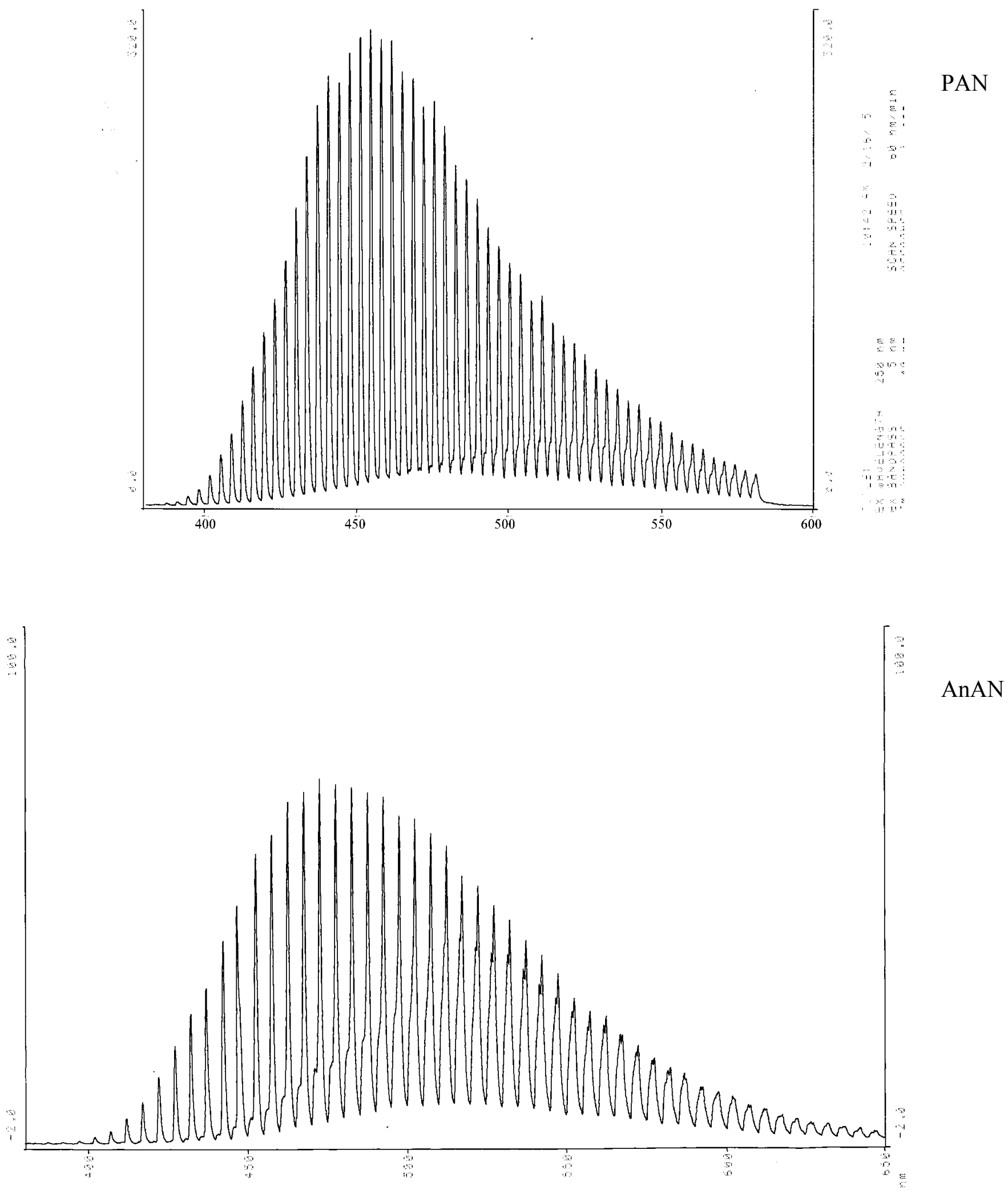


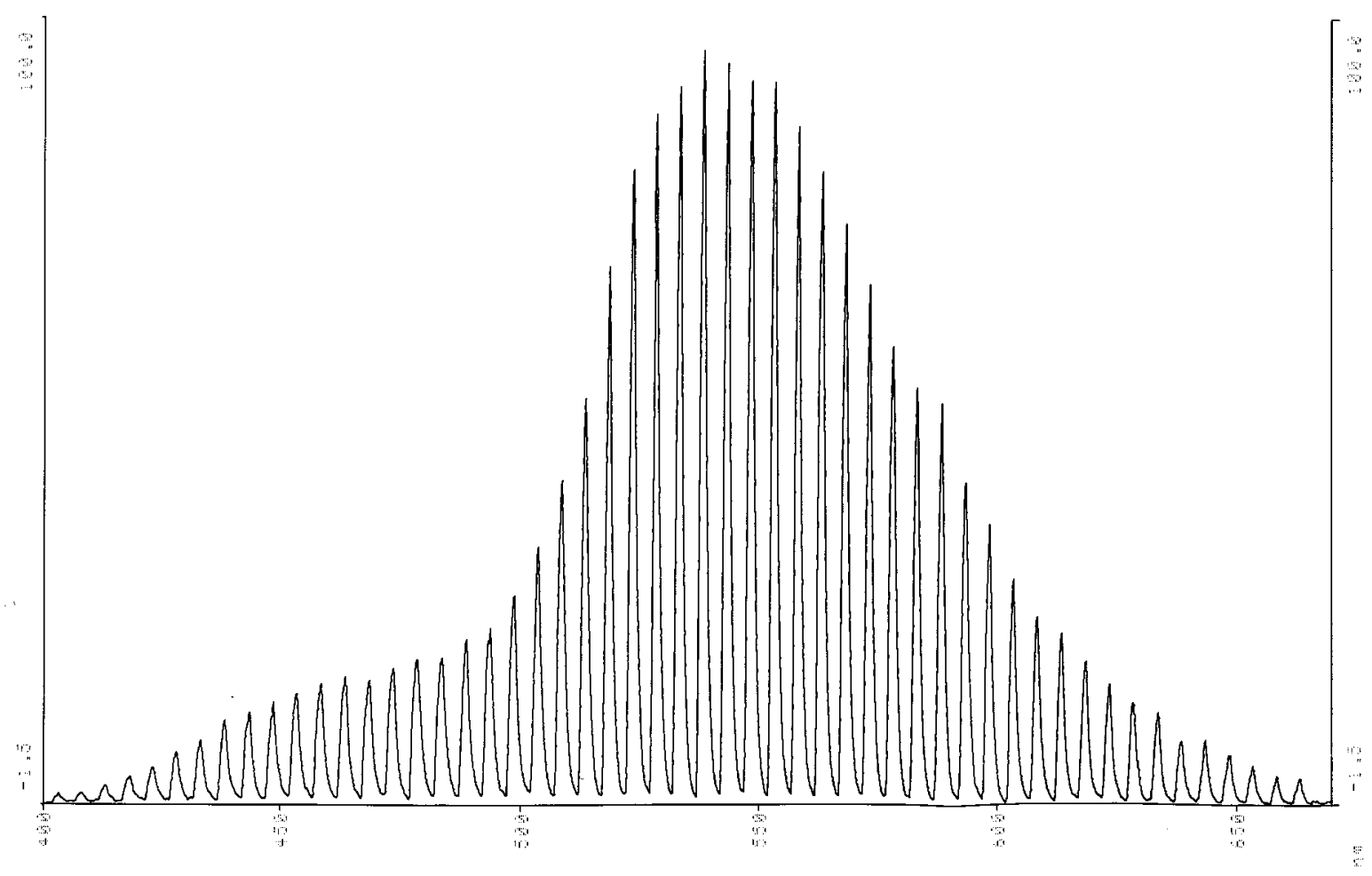

DMA-PAN

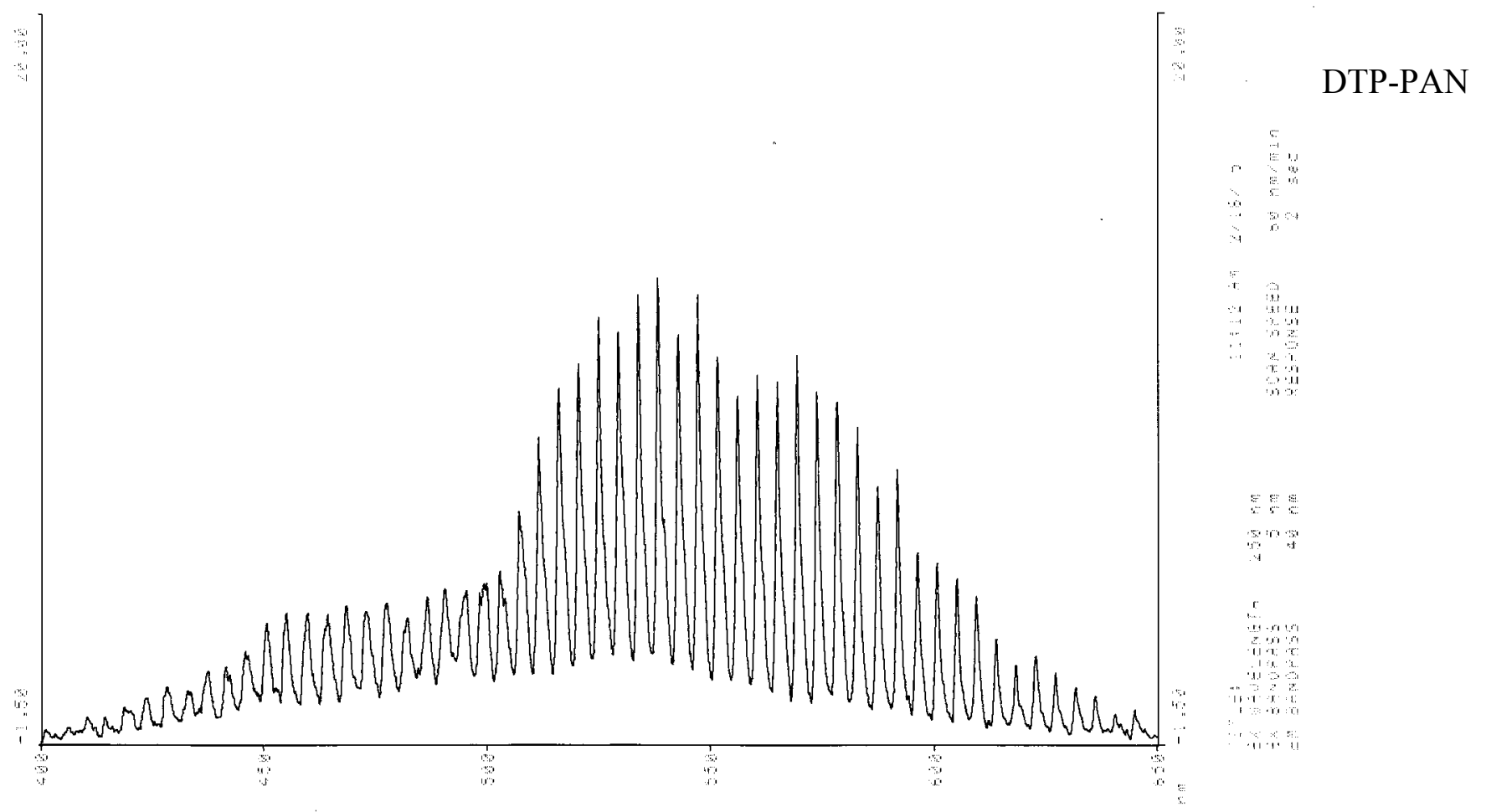





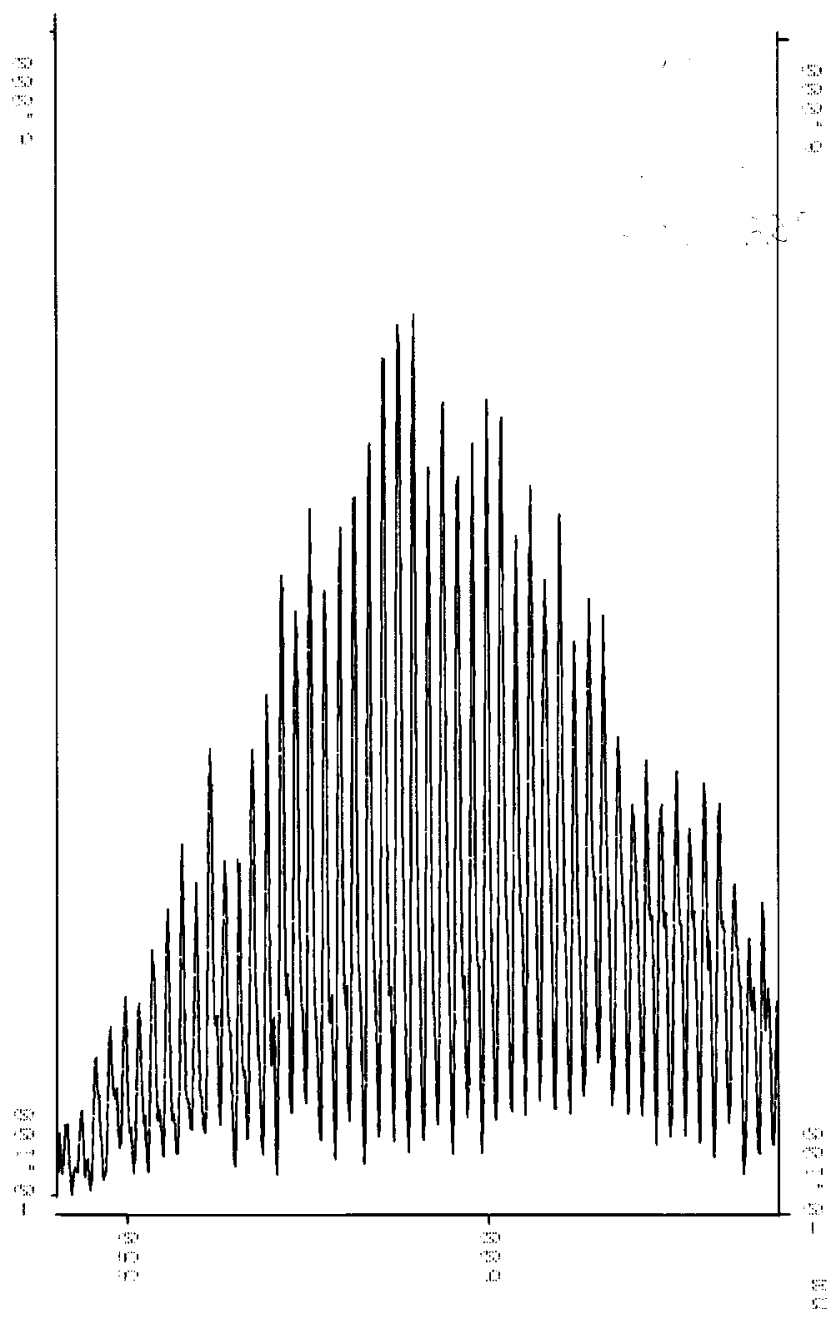

DMA-PEAN

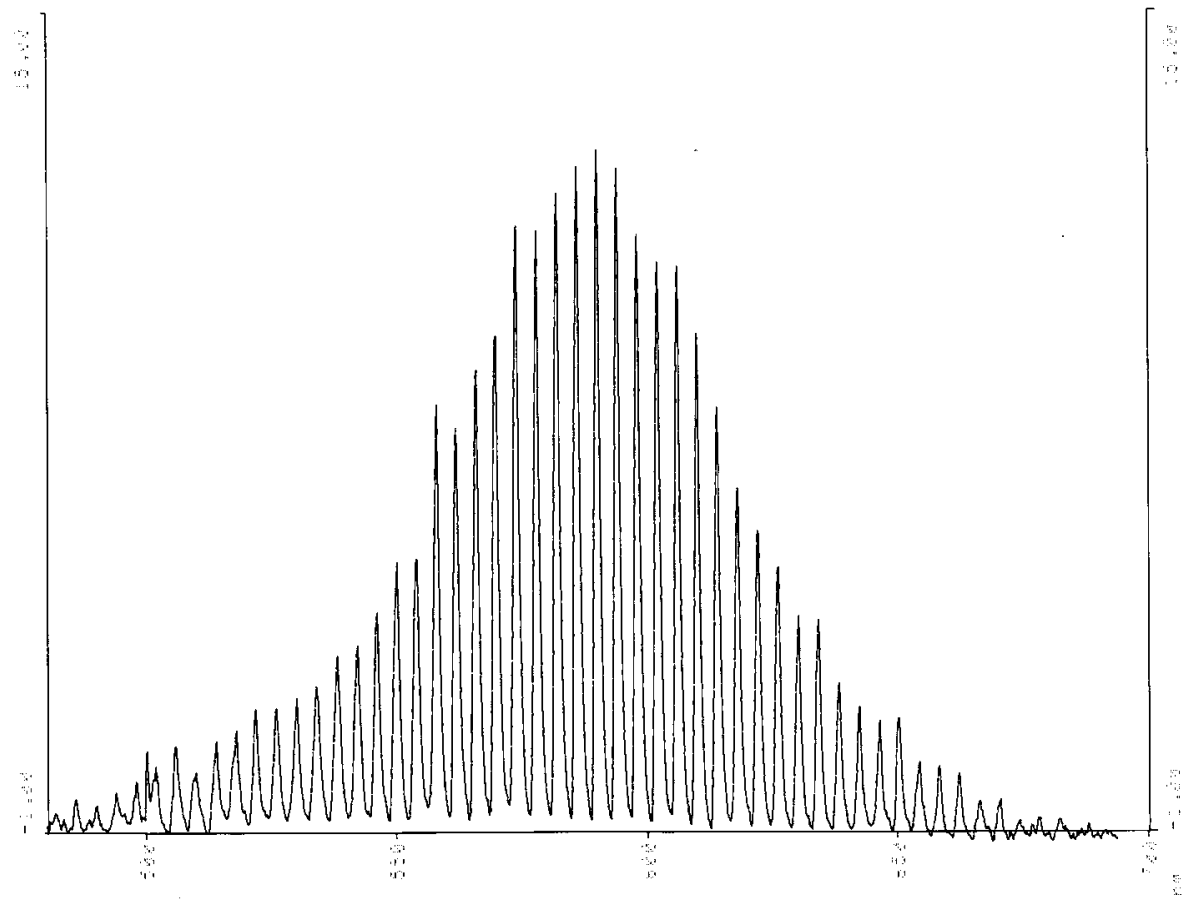

DTP-PEAN 


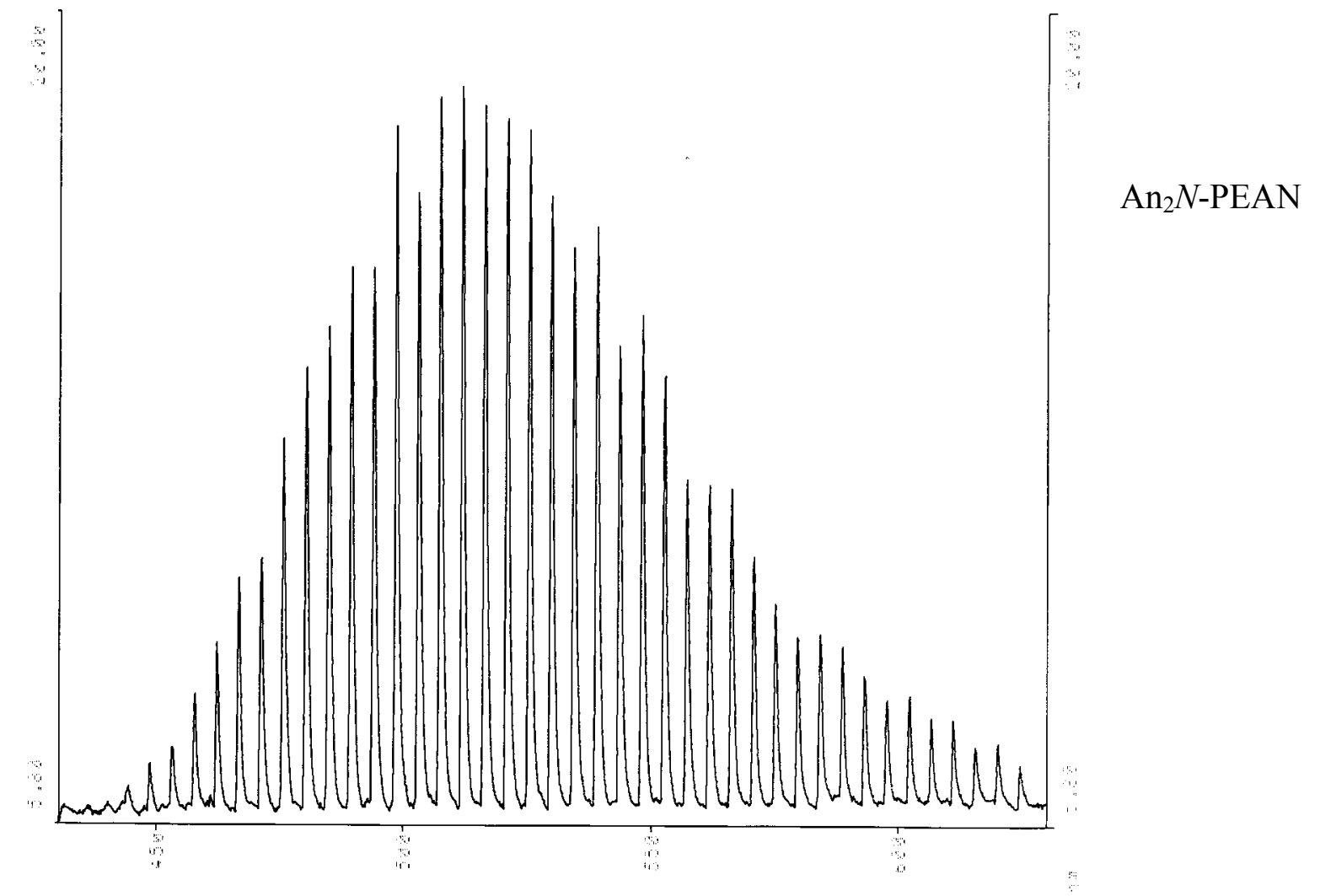


Cartessian coordinates of PANs PEANs

PAN Energy: -862.83 au; imaginary frequency: none.

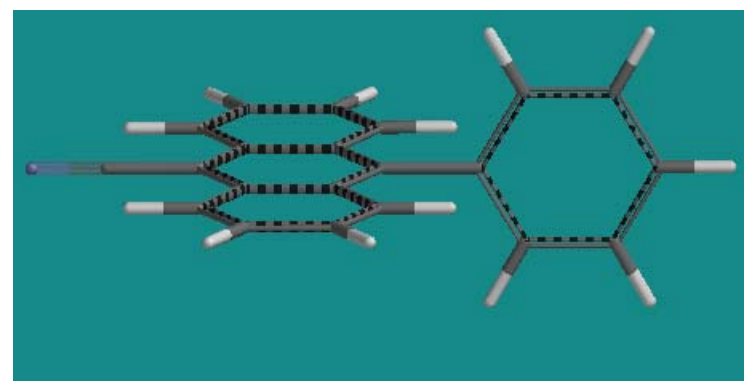

\begin{tabular}{|c|c|c|c|c|c|}
\hline & & & $\underset{x}{\text { Cartesian }}$ & $\underset{\mathrm{Y}}{\text { Coordinates }}$ & ingstroms ) \\
\hline & & Atom & $\mathrm{X}$ & $\mathrm{Y}$ & Z \\
\hline & & ------- & --ー--ー-----ー & ------------- & ------------ \\
\hline 1 & $\mathrm{H}$ & $\mathrm{H} 1$ & -4.6110737 & 0.0000000 & 2.4878323 \\
\hline 2 & $\mathrm{C}$ & $\mathrm{C} 1$ & -3.6678410 & 0.0000000 & 1.9489184 \\
\hline 3 & $\mathrm{H}$ & $\mathrm{H} 6$ & -2.4848340 & 0.0000000 & 3.7271195 \\
\hline 4 & $\mathrm{C}$ & $\mathrm{C} 6$ & -2.4857225 & 0.0000000 & 2.6415687 \\
\hline 5 & $\mathrm{C}$ & $\mathrm{C} 3$ & -2.4883079 & 0.0000000 & -0.1666718 \\
\hline 6 & $\mathrm{C}$ & $\mathrm{C} 5$ & -1.2341752 & 0.0000000 & 1.9550103 \\
\hline 7 & $\mathrm{C}$ & $\mathrm{C} 2$ & -3.6692497 & 0.0000000 & 0.5272722 \\
\hline 8 & $\mathrm{C}$ & $\mathrm{C} 4$ & -1.2275945 & 0.0000000 & 0.5129253 \\
\hline 9 & $\mathrm{C}$ & $\mathrm{C} 10$ & 0.0000000 & 0.0000000 & 2.6458643 \\
\hline 10 & $\mathrm{H}$ & $\mathrm{H} 2$ & -4.6140801 & 0.0000000 & -0.0088592 \\
\hline 11 & $\mathrm{H}$ & H3 & -2.4949749 & 0.0000000 & -1.2507650 \\
\hline 12 & $\mathrm{C}$ & $\mathrm{C9}$ & 1.2341752 & 0.0000000 & 1.9550103 \\
\hline 13 & $\mathrm{C}$ & C1 4 & 2.4857225 & 0.0000000 & 2.6415687 \\
\hline 14 & $\mathrm{C}$ & C8 & 1.2275945 & 0.0000000 & 0.5129253 \\
\hline 15 & $\mathrm{H}$ & $\mathrm{H} 11$ & 2.4949749 & 0.0000000 & -1.2507650 \\
\hline 16 & $\mathrm{C}$ & $\mathrm{C} 7$ & 0.0000000 & 0.0000000 & -0.1880106 \\
\hline 17 & $\mathrm{C}$ & C13 & 3.6678410 & 0.0000000 & 1.9489184 \\
\hline 18 & $\mathrm{H}$ & H1 4 & 2.4848340 & 0.0000000 & 3.7271195 \\
\hline 19 & $\mathrm{H}$ & $\mathrm{H} 13$ & 4.6110737 & 0.0000000 & 2.4878323 \\
\hline 20 & $\mathrm{C}$ & C12 & 3.6692497 & 0.0000000 & 0.5272722 \\
\hline 21 & $\mathrm{H}$ & $\mathrm{H} 12$ & 4.6140801 & 0.0000000 & -0.0088592 \\
\hline 22 & $\mathrm{C}$ & C11 & 2.4883079 & 0.0000000 & -0.1666718 \\
\hline 23 & $\mathrm{C}$ & C15 & 0.0000000 & 0.0000000 & -1.6846210 \\
\hline 24 & $\mathrm{C}$ & C1 6 & 0.0000000 & 0.0000000 & -4.4950487 \\
\hline 25 & $\mathrm{C}$ & C1 7 & 0.0000000 & 1.2071950 & -2.3984977 \\
\hline 26 & $\mathrm{C}$ & C18 & 0.0000000 & -1.2071950 & -2.3984977 \\
\hline 27 & $\mathrm{C}$ & C19 & 0.0000000 & -1.2068222 & -3.7939135 \\
\hline 28 & $\mathrm{C}$ & $\mathrm{C} 20$ & 0.0000000 & 1.2068222 & -3.7939135 \\
\hline 29 & $\mathrm{H}$ & $\mathrm{H} 4$ & 0.0000000 & 2.1488654 & -1.8560370 \\
\hline 30 & $\mathrm{H}$ & H5 & 0.0000000 & -2.1488654 & -1.8560370 \\
\hline 31 & $\mathrm{H}$ & $\mathrm{H} 7$ & 0.0000000 & -2.1506575 & -4.3325427 \\
\hline 32 & $\mathrm{H}$ & H8 & 0.0000000 & 2.1506575 & -4.3325427 \\
\hline 33 & $\mathrm{H}$ & H9 & 0.0000000 & 0.0000000 & -5.5816533 \\
\hline 34 & $\mathrm{C}$ & $\mathrm{C} 21$ & 0.0000000 & 0.0000000 & 4.0758573 \\
\hline 35 & $\mathrm{~N}$ & N1 & 0.0000000 & 0.0000000 & 5.2408925 \\
\hline
\end{tabular}

Point Group $=\mathrm{CNV}$ Order $=2$ Nsymop $=4$ 
AnAN Energy: -977.35 au; Imaginary frequeny: none

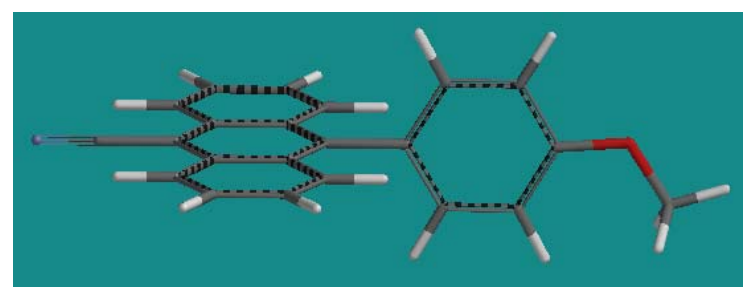

Atom

Cartesian Coordinates (Angstroms) $\mathrm{X}$ Y $\mathrm{Z}$

\begin{tabular}{|c|c|c|c|c|c|}
\hline 1 & $\mathrm{H}$ & $\mathrm{H} 1$ & -3.1954557 & 0.1129542 & 4.6108876 \\
\hline 2 & $\mathrm{C}$ & $\mathrm{C} 1$ & -2.6581511 & 0.0694235 & 3.6676797 \\
\hline 3 & $\mathrm{H}$ & H6 & -4.4305216 & 0.2122569 & 2.4845602 \\
\hline 4 & C & $\mathrm{C} 6$ & -3.3484616 & 0.1251213 & 2.4855208 \\
\hline 5 & $\mathrm{C}$ & C3 & -0.5495881 & -0.0998499 & 2.4879766 \\
\hline 6 & $\mathrm{C}$ & $\mathrm{C} 5$ & -2.6640826 & 0.0702126 & 1.2340515 \\
\hline 7 & $\mathrm{C}$ & $\mathrm{C} 2$ & -1.2411340 & -0.0451919 & 3.6690354 \\
\hline 8 & $\mathrm{C}$ & $\mathrm{C} 4$ & -1.2265521 & -0.0456863 & 1.2271370 \\
\hline 9 & $\mathrm{C}$ & $\mathrm{C} 10$ & -3.3528266 & 0.1264366 & 0.0000000 \\
\hline 10 & $\mathrm{H}$ & $\mathrm{H} 2$ & -0.7067401 & -0.0894311 & 4.6138714 \\
\hline 11 & $\mathrm{H}$ & H3 & 0.5309595 & -0.1866119 & 2.4934228 \\
\hline 12 & C & C9 & -2.6640826 & 0.0702126 & -1.2340515 \\
\hline 13 & C & C14 & -3.3484616 & 0.1251213 & -2.4855208 \\
\hline 14 & $\mathrm{C}$ & $\mathrm{C} 8$ & -1.2265521 & -0.0456863 & -1.2271370 \\
\hline 15 & $\mathrm{H}$ & $\mathrm{H} 11$ & 0.5309595 & -0.1866119 & -2.4934228 \\
\hline 16 & $\mathrm{C}$ & $\mathrm{C7}$ & -0.5258845 & -0.0994578 & 0.0000000 \\
\hline 17 & $\mathrm{C}$ & $\mathrm{C} 13$ & -2.6581511 & 0.0694235 & -3.6676797 \\
\hline 18 & $\mathrm{H}$ & $\mathrm{H} 14$ & -4.4305216 & 0.2122569 & -2.4845602 \\
\hline 19 & $\mathrm{H}$ & $\mathrm{H} 13$ & -3.1954557 & 0.1129542 & -4.6108876 \\
\hline 20 & $\mathrm{C}$ & $\mathrm{C} 12$ & -1.2411340 & -0.0451919 & -3.6690354 \\
\hline 21 & $\mathrm{H}$ & $\mathrm{H} 12$ & -0.7067401 & -0.0894311 & -4.6138714 \\
\hline 22 & C & $\mathrm{C} 11$ & -0.5495881 & -0.0998499 & -2.4879766 \\
\hline 23 & $\mathrm{C}$ & C15 & 0.9658454 & -0.2114012 & 0.0000000 \\
\hline 24 & $\mathrm{C}$ & C16 & 3.7796317 & -0.4137862 & 0.0000000 \\
\hline 25 & $\mathrm{C}$ & $\mathrm{C} 17$ & 1.5997657 & -1.4657266 & 0.0000000 \\
\hline 26 & $\mathrm{C}$ & $\mathrm{C} 18$ & 1.7709086 & 0.9317761 & 0.0000000 \\
\hline 27 & $\mathrm{C}$ & C19 & 3.1655924 & 0.8438361 & 0.0000000 \\
\hline 28 & $\mathrm{C}$ & $\mathrm{C} 20$ & 2.9846386 & -1.5695328 & 0.0000000 \\
\hline 29 & $\mathrm{H}$ & $\mathrm{H} 4$ & 0.9972877 & -2.3701327 & 0.0000000 \\
\hline 30 & $\mathrm{H}$ & H5 & 1.3053571 & 1.9137829 & 0.0000000 \\
\hline 31 & $\mathrm{H}$ & $\mathrm{H} 7$ & 3.7543439 & 1.7543373 & 0.0000000 \\
\hline 32 & $\mathrm{H}$ & $\mathrm{H} 8$ & 3.4762883 & -2.5373370 & 0.0000000 \\
\hline 33 & $\mathrm{C}$ & $\mathrm{C} 21$ & -4.7778696 & 0.2453459 & 0.0000000 \\
\hline 34 & $\mathrm{~N}$ & N1 & -5.9388736 & 0.3428143 & 0.0000000 \\
\hline 35 & 0 & 01 & 5.1271154 & -0.6222954 & 0.0000000 \\
\hline 36 & $\mathrm{C}$ & $\mathrm{C} 22$ & 5.9824354 & 0.5106872 & 0.0000000 \\
\hline 37 & $\mathrm{H}$ & $\mathrm{H} 9$ & 5.8327374 & 1.1288779 & -0.8950957 \\
\hline 38 & $\mathrm{H}$ & $\mathrm{H} 1 \mathrm{O}$ & 7.0002239 & 0.1165027 & 0.0000000 \\
\hline 39 & $\mathrm{H}$ & H15 & 5.8327374 & 1.1288779 & 0.8950957 \\
\hline
\end{tabular}

Point Group $=\mathrm{CS}$ Order $=1$ Nsymop $=2$ 


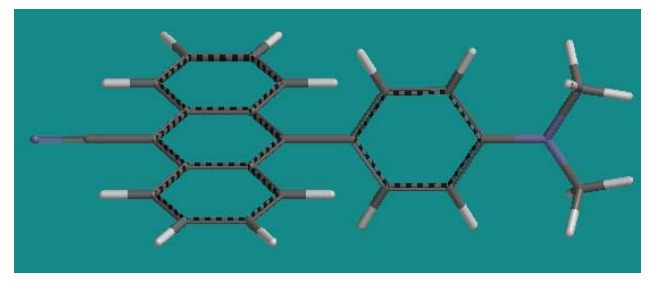

\begin{tabular}{|c|c|c|c|c|c|}
\hline & & Atom & $\begin{array}{l}\text { Cartesian } \\
\quad \mathrm{X}\end{array}$ & $\begin{array}{c}\text { Coordinates } \\
\text { Y }\end{array}$ & $\begin{array}{c}\text { (Angstroms) } \\
Z\end{array}$ \\
\hline 1 & $\mathrm{H}$ & $\mathrm{H} 1$ & -4.6110793 & 0.0000000 & -3.7649274 \\
\hline 2 & $\mathrm{C}$ & $\mathrm{C} 1$ & -3.6676528 & 0.0000000 & -3.2261087 \\
\hline 3 & $\mathrm{H}$ & H 6 & -2.4854988 & 0.0000000 & -5.0048750 \\
\hline 4 & C & $\mathrm{C} 6$ & -2.4857915 & 0.0000000 & -3.9192780 \\
\hline 5 & $\mathrm{C}$ & $\mathrm{C} 3$ & -2.4871280 & 0.0000000 & -1.1114307 \\
\hline 6 & $\mathrm{C}$ & $\mathrm{C} 5$ & -1.2340513 & 0.0000000 & -3.2331704 \\
\hline 7 & C & $\mathrm{C} 2$ & -3.6685865 & 0.0000000 & -1.8044913 \\
\hline 8 & C & $\mathrm{C} 4$ & -1.2266824 & 0.0000000 & -1.7911108 \\
\hline 9 & C & $\mathrm{C} 10$ & 0.0000000 & 0.0000000 & -3.9247342 \\
\hline 10 & $\mathrm{H}$ & $\mathrm{H} 2$ & -4.6133730 & 0.0000000 & -1.2680546 \\
\hline 11 & $\mathrm{H}$ & H3 & -2.4900556 & 0.0000000 & -0.0273947 \\
\hline 12 & C & C9 & 1.2340513 & 0.0000000 & -3.2331704 \\
\hline 13 & $\mathrm{C}$ & $\mathrm{C} 14$ & 2.4857915 & 0.0000000 & -3.9192780 \\
\hline 14 & C & $\mathrm{C} 8$ & 1.2266824 & 0.0000000 & -1.7911108 \\
\hline 15 & $\mathrm{H}$ & $\mathrm{H} 11$ & 2.4900556 & 0.0000000 & -0.0273947 \\
\hline 16 & C & $\mathrm{C} 7$ & 0.0000000 & 0.0000000 & -1.0863640 \\
\hline 17 & C & C13 & 3.6676528 & 0.0000000 & -3.2261087 \\
\hline 18 & $\mathrm{H}$ & $\mathrm{H} 14$ & 2.4854988 & 0.0000000 & -5.0048750 \\
\hline 19 & $\mathrm{H}$ & H13 & 4.6110793 & 0.0000000 & -3.7649274 \\
\hline 20 & $\mathrm{C}$ & C12 & 3.6685865 & 0.0000000 & -1.8044913 \\
\hline 21 & $\mathrm{H}$ & $\mathrm{H} 12$ & 4.6133730 & 0.0000000 & -1.2680546 \\
\hline 22 & C & $\mathrm{C} 11$ & 2.4871280 & 0.0000000 & -1.1114307 \\
\hline 23 & C & C15 & 0.0000000 & 0.0000000 & 0.4082498 \\
\hline 24 & C & C16 & 0.0000000 & 0.0000000 & 3.2652776 \\
\hline 25 & $\mathrm{C}$ & $\mathrm{C} 17$ & 0.0000000 & -1.1969331 & 1.1371102 \\
\hline 26 & $\mathrm{C}$ & $\mathrm{C} 18$ & 0.0000000 & 1.1969331 & 1.1371102 \\
\hline 27 & $\mathrm{C}$ & C19 & 0.0000000 & 1.2065990 & 2.5280954 \\
\hline 28 & $\mathrm{C}$ & $\mathrm{C} 20$ & 0.0000000 & -1.2065990 & 2.5280954 \\
\hline 29 & $\mathrm{H}$ & $\mathrm{H} 4$ & 0.0000000 & -2.1460231 & 0.6068002 \\
\hline 30 & $\mathrm{H}$ & H5 & 0.0000000 & 2.1460231 & 0.6068002 \\
\hline 31 & $\mathrm{H}$ & $\mathrm{H} 7$ & 0.0000000 & 2.1614100 & 3.0400575 \\
\hline 32 & $\mathrm{H}$ & $\mathrm{H} 8$ & 0.0000000 & -2.1614100 & 3.0400575 \\
\hline 33 & C & $\mathrm{C} 21$ & 0.0000000 & 0.0000000 & -5.3545776 \\
\hline 34 & $\mathrm{~N}$ & N1 & 0.0000000 & 0.0000000 & -6.5197618 \\
\hline 35 & $\mathrm{~N}$ & N2 & 0.0000000 & 0.0000000 & 4.6491212 \\
\hline 36 & C & $\mathrm{C} 22$ & 0.0000000 & -1.2559964 & 5.3753363 \\
\hline 37 & $\mathrm{H}$ & $\mathrm{H} 10$ & 0.8887045 & -1.8634723 & 5.1487450 \\
\hline 38 & $\mathrm{H}$ & H15 & -0.8887045 & -1.8634723 & 5.1487450 \\
\hline 39 & $\mathrm{H}$ & H1 6 & 0.0000000 & -1.0513950 & 6.4473464 \\
\hline 40 & $\mathrm{C}$ & C23 & 0.0000000 & 1.2559964 & 5.3753363 \\
\hline 41 & $\mathrm{H}$ & H9 & 0.0000000 & 1.0513950 & 6.4473464 \\
\hline 42 & $\mathrm{H}$ & $\mathrm{H} 17$ & -0.8887045 & 1.8634723 & 5.1487450 \\
\hline 43 & $\mathrm{H}$ & H18 & 0.8887045 & 1.8634723 & 5.1487450 \\
\hline
\end{tabular}

Point Group $=\mathrm{CNV}$ Order $=2$ Nsymop $=4$ 


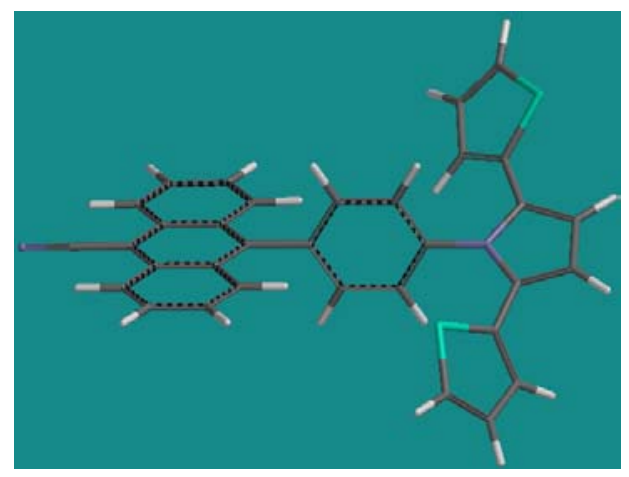

\begin{tabular}{|c|c|c|c|c|}
\hline & Atom & $\begin{array}{l}\text { Cartesian } \\
\quad \mathrm{X}\end{array}$ & $\begin{array}{c}\text { Coordinates } \\
\text { Y }\end{array}$ & $\begin{array}{c}\text { gstroms ) } \\
z\end{array}$ \\
\hline & & -------- & ------- & \\
\hline 1 & $\mathrm{H} 1$ & 5.0199599 & -4.2309093 & -1.6767573 \\
\hline 2 & $\mathrm{C} 1$ & 4.4675908 & -3.3629618 & -1.3279869 \\
\hline 3 & $\mathrm{H} 6$ & 6.2267846 & -2.2114685 & -0.9478658 \\
\hline 4 & $\mathrm{C} 6$ & 5.1418581 & -2.2409383 & -0.9236299 \\
\hline 5 & $\mathrm{C} 3$ & 2.3352219 & -2.3147609 & -0.8553166 \\
\hline 6 & $\mathrm{C} 5$ & 4.4366995 & -1.0891504 & -0.4608401 \\
\hline 7 & $\mathrm{C} 2$ & 3.0464780 & -3.4012226 & -1.2908834 \\
\hline 8 & $\mathrm{C} 4$ & 2.9947818 & -1.1173180 & -0.4286965 \\
\hline 9 & $\mathrm{C} 10$ & 5.1090770 & 0.0784007 & -0.0306892 \\
\hline 10 & $\mathrm{H} 2$ & 2.5251882 & -4.2996821 & -1.6091218 \\
\hline 11 & H3 & 1.2521056 & -2.3493610 & -0.8239262 \\
\hline $12 c$ & C9 & 4.4007574 & 1.2136097 & 0.4276122 \\
\hline $13 c$ & $\mathrm{C} 14$ & 5.0695100 & 2.3961143 & 0.8660203 \\
\hline 14 & $\mathrm{C} 8$ & 2.9588026 & 1.1761926 & 0.4494195 \\
\hline 15 & $\mathrm{H} 11$ & 1.1772409 & 2.3260024 & 0.9175727 \\
\hline 16 & $\mathrm{C} 7$ & 2.2765528 & 0.0141207 & 0.0213073 \\
\hline 17 & $\mathrm{C} 13$ & 4.3600611 & 3.4848796 & 1.3001770 \\
\hline 18 & $\mathrm{H} 14$ & 6.1547810 & 2.4168878 & 0.8484888 \\
\hline 19 & $\mathrm{H} 13$ & 4.8851402 & 4.3765671 & 1.6308879 \\
\hline 20 & C12 & 2.9383860 & 3.4574474 & 1.3184888 \\
\hline 21 & $\mathrm{H} 12$ & 2.3888712 & 4.3295885 & 1.6613439 \\
\hline 22 & $\mathrm{C} 11$ & 2.2610598 & 2.3408505 & 0.9056035 \\
\hline 23 & C15 & 0.7795076 & -0.0148146 & 0.0407870 \\
\hline 24 & C16 & -2.0278705 & -0.0493368 & 0.0791163 \\
\hline 25 & C17 & 0.0838977 & -0.3338961 & 1.2159671 \\
\hline 26 & $\mathrm{C} 18$ & 0.0448944 & 0.2839267 & -1.1157962 \\
\hline 27 & C19 & -1.3488040 & 0.2619542 & -1.1019721 \\
\hline 28 & $\mathrm{C} 20$ & -1.3093548 & -0.3471551 & 1.2397966 \\
\hline 29 & $\mathrm{H} 4$ & 0.6395380 & -0.5668923 & 2.1197953 \\
\hline 30 & H5 & 0.5702284 & 0.5351263 & -2.0328198 \\
\hline 31 & $\mathrm{H} 7$ & -1.9142450 & 0.4934106 & -1.9989010 \\
\hline 32 & $\mathrm{H} 8$ & -1.8441622 & -0.5869368 & 2.1530227 \\
\hline 33 & C21 & 6.5388876 & 0.1115847 & -0.0594493 \\
\hline 34 & N1 & 7.7032119 & 0.1384774 & -0.0832976 \\
\hline 35 & N2 & -3.4570100 & -0.0652174 & 0.0982524 \\
\hline
\end{tabular}




$\begin{array}{lllrrr}36 & \text { C } & \text { C22 } & -4.2553655 & -1.1875350 & 0.3253834 \\ 37 & \text { C } & \text { C23 } & -5.5785649 & -0.7750772 & 0.2607221 \\ 38 & \text { C } & \text { C27 } & -4.2803292 & 1.0420425 & -0.1121137 \\ 39 & \text { C } & \text { C28 } & -5.5936908 & 0.6085435 & -0.0088182 \\ 40 & \text { H } & \text { H34 } & -6.4343912 & -1.4267939 & 0.3724279 \\ 41 & \text { H } & \text { H35 } & -6.4607295 & 1.2489726 & -0.0924195 \\ 42 & \text { C } & \text { C24 } & -3.8203675 & 2.4000268 & -0.3655883 \\ 43 & \text { C } & \text { C25 } & -4.3798001 & 3.2873418 & -1.2586598 \\ 44 & \text { C } & \text { C26 } & -3.8151027 & 4.5938220 & -1.2028569 \\ 45 & \text { C } & \text { C29 } & -2.8248243 & 4.7032885 & -0.2662846 \\ 46 & \text { S } & \text { S2 } & -2.5729472 & 3.2082335 & 0.5763644 \\ 47 & \text { H } & \text { H15 } & -5.1672171 & 2.9958516 & -1.9451901 \\ 48 & \text { H } & \text { H16 } & -4.1223939 & 5.4112779 & -1.8462495 \\ 49 & \text { H } & \text { H19 } & -2.2110292 & 5.5636546 & -0.0344118 \\ 50 & \text { C } & \text { C31 } & -3.7381007 & -2.5293721 & 0.5433515 \\ 51 & \text { C } & \text { C32 } & -2.6708851 & -3.1884288 & -0.0268174 \\ 52 & \text { C } & \text { C33 } & -2.5257309 & -4.5325954 & 0.4242192 \\ 53 & \text { C } & \text { C34 } & -3.4802865 & -4.8979744 & 1.3328982 \\ 54 & \text { S } & \text { S1 } & -4.5686194 & -3.5908432 & 1.6705973 \\ 55 & \text { H } & \text { H24 } & -2.0259472 & -2.7312532 & -0.7680249 \\ 56 & \text { H } & \text { H26 } & -1.7481206 & -5.2027697 & 0.0730808 \\ 57 & \text { H } & \text { H28 } & -3.6111839 & -5.8535314 & 1.8226804\end{array}$

Point Group $=\mathrm{C} 1$ Order $=1$ Nsymop $=1$

\section{$\mathbf{A n}_{2} \mathbf{N}$-PAN Energy: -1609.18 au; imaginary frequency: none}

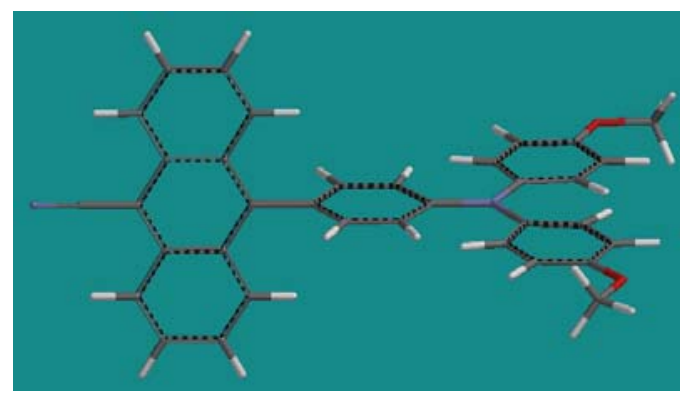

Atom

Cartesian Coordinates (Angstroms)

$\begin{array}{rll} & & \text { Atom } \\ & & \\ 1 & \text { H } & \text { H1 } \\ 2 & \text { C } & \text { C1 } \\ 3 & \text { H } & \text { H6 } \\ 4 & \text { C } & \text { C6 } \\ 5 & \text { C } & \text { C3 } \\ 6 & \text { C } & \text { C5 } \\ 7 & \text { C } & \text { C2 } \\ 8 & \text { C } & \text { C4 } \\ 9 & \text { C } & \text { C10 } \\ 10 & \text { H } & \text { H2 } \\ 11 & \text { H } & \text { H3 } \\ 12 & \text { C } & \text { C9 }\end{array}$

$\mathrm{X}$

Y

Z

$\begin{array}{lll}-6.3250019 & -0.2285093 & 4.6114787 \\ -5.7863217 & -0.2126868 & 3.6681311 \\ -7.5643442 & -0.2607495 & 2.4845755 \\ -6.4790714 & -0.2307955 & 2.4860985 \\ -3.6712339 & -0.1539978 & 2.4884451 \\ -5.7927635 & -0.2113242 & 1.2344567 \\ -4.3648190 & -0.1735815 & 3.6694931 \\ -4.3510965 & -0.1716682 & 1.2279929 \\ -6.4835414 & -0.2303809 & 0.0000000 \\ -3.8285341 & -0.1596377 & 4.6141893 \\ -2.5873747 & -0.1248112 & 2.4935453 \\ -5.7927635 & -0.2113242 & -1.2344567\end{array}$




\begin{tabular}{|c|c|c|c|c|c|}
\hline 13 & $\mathrm{C}$ & C14 & -6.4790714 & -0.2307955 & -2.4860985 \\
\hline 14 & C & $\mathrm{C} 8$ & -4.3510965 & -0.1716682 & -1.2279929 \\
\hline 15 & $\mathrm{H}$ & H11 & -2.5873747 & -0.1248112 & -2.4935453 \\
\hline 16 & $\mathrm{C}$ & $\mathrm{C} 7$ & -3.6496536 & -0.1531754 & 0.0000000 \\
\hline 17 & $\mathrm{C}$ & C13 & -5.7863217 & -0.2126868 & -3.6681311 \\
\hline 18 & $\mathrm{H}$ & $\mathrm{H} 14$ & -7.5643442 & -0.2607495 & -2.4845755 \\
\hline 19 & $\mathrm{H}$ & H13 & -6.3250019 & -0.2285093 & -4.6114787 \\
\hline 20 & $\mathrm{C}$ & $\mathrm{C} 12$ & -4.3648190 & -0.1735815 & -3.6694931 \\
\hline 21 & $\mathrm{H}$ & $\mathrm{H} 12$ & -3.8285341 & -0.1596377 & -4.6141893 \\
\hline 22 & $\mathrm{C}$ & $\mathrm{C} 11$ & -3.6712339 & -0.1539978 & -2.4884451 \\
\hline 23 & $\mathrm{C}$ & C15 & -2.1582682 & -0.1182421 & 0.0000000 \\
\hline 24 & $\mathrm{C}$ & C16 & 0.8368113 & -0.0537032 & 0.0000000 \\
\hline 25 & $\mathrm{C}$ & C17 & -1.3712292 & -1.2630695 & 0.0000000 \\
\hline 26 & C & C18 & -1.4213507 & 1.0592708 & 0.0000000 \\
\hline 27 & $\mathrm{C}$ & C19 & -0.0267179 & 1.0809653 & 0.0000000 \\
\hline 28 & $\mathrm{C}$ & $\mathrm{C} 20$ & 0.0224177 & -1.2234238 & 0.0000000 \\
\hline 29 & $\mathrm{H}$ & $\mathrm{H} 4$ & -1.8379421 & -2.2449522 & 0.0000000 \\
\hline 30 & $\mathrm{H}$ & H5 & -1.9303434 & 2.0200211 & 0.0000000 \\
\hline 31 & $\mathrm{H}$ & $\mathrm{H} 7$ & 0.3048784 & 2.0818726 & 0.0000000 \\
\hline 32 & $\mathrm{H}$ & H8 & 0.3999223 & -2.2073706 & 0.0000000 \\
\hline 33 & C & $\mathrm{C} 21$ & -7.9131581 & -0.2711466 & 0.0000000 \\
\hline 34 & $\mathrm{~N}$ & N1 & -9.0776924 & -0.3043169 & 0.0000000 \\
\hline 35 & $\mathrm{~N}$ & N2 & 2.3292853 & -0.0270704 & 0.0000000 \\
\hline 36 & C & $\mathrm{C} 22$ & 3.0620037 & 1.2975506 & 0.0000000 \\
\hline 37 & $\mathrm{C}$ & C23 & 4.5016938 & 3.9035432 & 0.0000000 \\
\hline 38 & C & C24 & 4.4779438 & 1.4723196 & 0.0000000 \\
\hline 39 & C & $\mathrm{C} 25$ & 2.4607500 & 2.5841577 & 0.0000000 \\
\hline 40 & $\mathrm{C}$ & C26 & 3.1184197 & 3.8185033 & 0.0000000 \\
\hline 41 & C & $\mathrm{C} 27$ & 5.1618628 & 2.6824755 & 0.0000000 \\
\hline 42 & $\mathrm{H}$ & H9 & 5.1761629 & 0.6816069 & 0.0000000 \\
\hline 43 & $\mathrm{H}$ & $\mathrm{H} 10$ & 1.4200539 & 2.7547804 & 0.0000000 \\
\hline 44 & $\mathrm{H}$ & H1 5 & 2.4939921 & 4.7052849 & 0.0000000 \\
\hline 45 & $\mathrm{H}$ & H1 6 & 6.2476711 & 2.6641639 & 0.0000000 \\
\hline 46 & $\mathrm{C}$ & $\mathrm{C} 28$ & 3.1106852 & -1.3244051 & 0.0000000 \\
\hline 47 & $\mathrm{C}$ & C29 & 4.6602195 & -3.8659753 & 0.0000000 \\
\hline 48 & C & C30 & 2.5594675 & -2.6416308 & 0.0000000 \\
\hline 49 & $\mathrm{C}$ & C31 & 4.5246833 & -1.4400782 & 0.0000000 \\
\hline 50 & C & C32 & 5.2717724 & -2.6223642 & 0.0000000 \\
\hline 51 & C & C33 & 3.2725273 & -3.8348331 & 0.0000000 \\
\hline 52 & $\mathrm{H}$ & H1 8 & 1.5270899 & -2.8596032 & 0.0000000 \\
\hline 53 & $\mathrm{H}$ & H1 9 & 5.1856568 & -0.6188064 & 0.0000000 \\
\hline 54 & $\mathrm{H}$ & $\mathrm{H} 2 \mathrm{O}$ & 6.3516248 & -2.5195975 & 0.0000000 \\
\hline 55 & $\mathrm{H}$ & H21 & 2.7185145 & -4.7688152 & 0.0000000 \\
\hline 56 & 0 & 01 & 5.2774713 & -5.0787212 & 0.0000000 \\
\hline 57 & 0 & $\mathrm{O} 2$ & 5.2496176 & 5.0403730 & 0.0000000 \\
\hline 58 & C & C34 & 6.6972901 & -5.0917728 & 0.0000000 \\
\hline 59 & $\mathrm{H}$ & H2 2 & 7.1043514 & -4.6023435 & -0.8947189 \\
\hline 60 & $\mathrm{H}$ & H23 & 6.9885448 & -6.1436110 & 0.0000000 \\
\hline 61 & $\mathrm{H}$ & H2 4 & 7.1043514 & -4.6023435 & 0.8947189 \\
\hline 62 & C & C35 & 4.5591036 & 6.2809182 & 0.0000000 \\
\hline 63 & $\mathrm{H}$ & $\mathrm{H} 17$ & 3.9324759 & 6.3926071 & -0.8947971 \\
\hline 64 & $\mathrm{H}$ & H2 5 & 5.3292267 & 7.0542544 & 0.0000000 \\
\hline 65 & $\mathrm{H}$ & $\mathrm{H} 26$ & 3.9324759 & 6.3926071 & 0.8947971 \\
\hline
\end{tabular}

Point Group $=$ CS Order $=1$ Nsymop $=2$ 
PEAN Energy:-938.99 au; Imaginary frequency: none

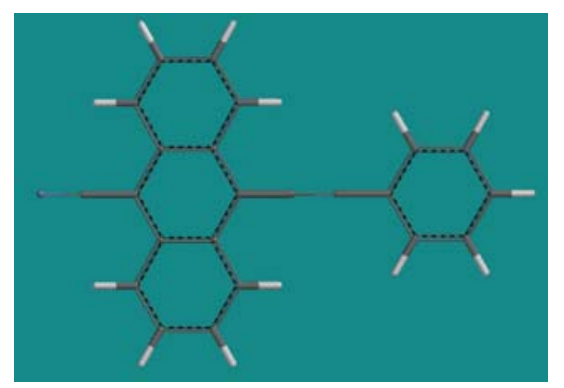

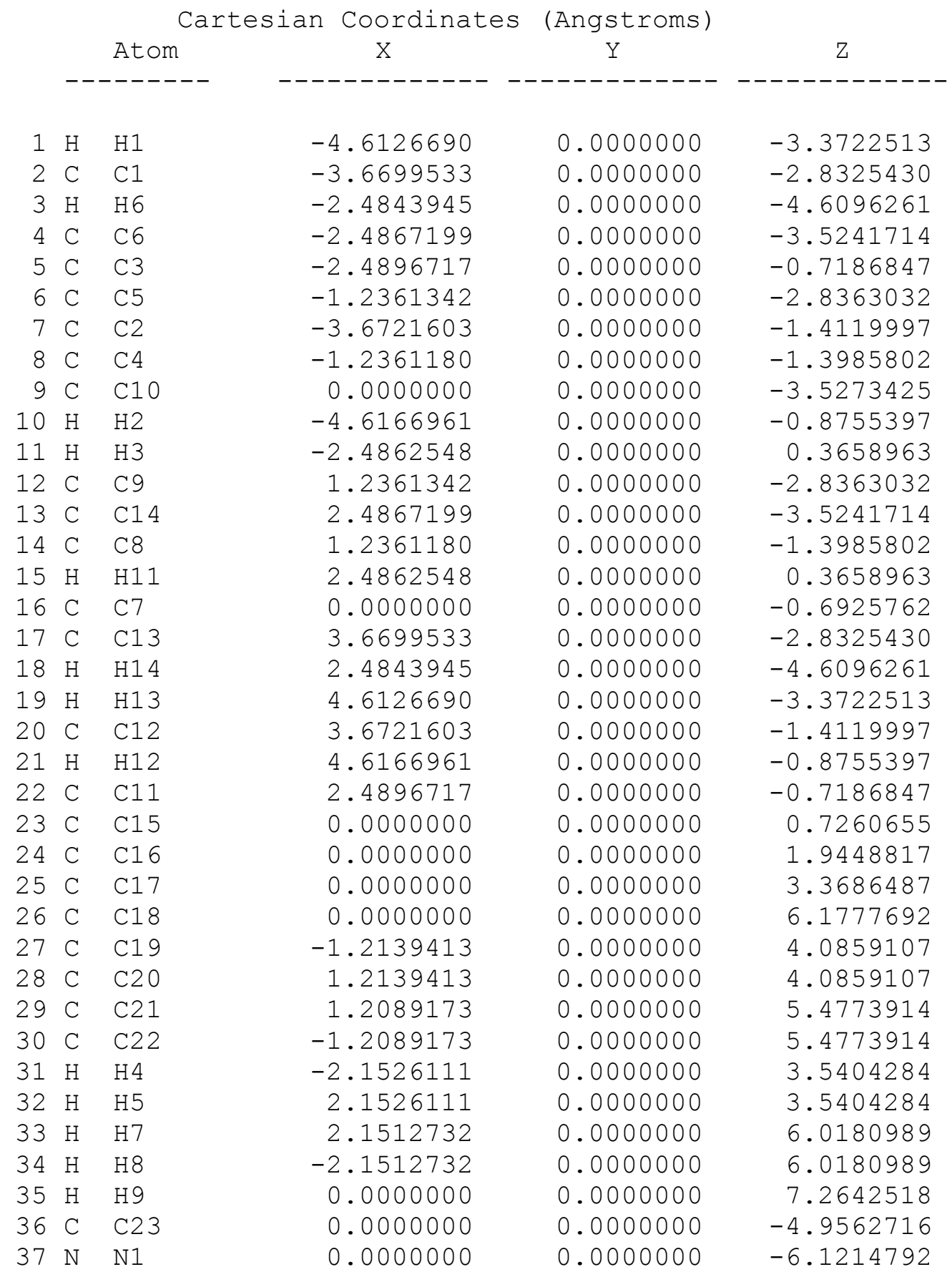


AnEAN

Energy: -1053.21 au; Imaginary frequency: none

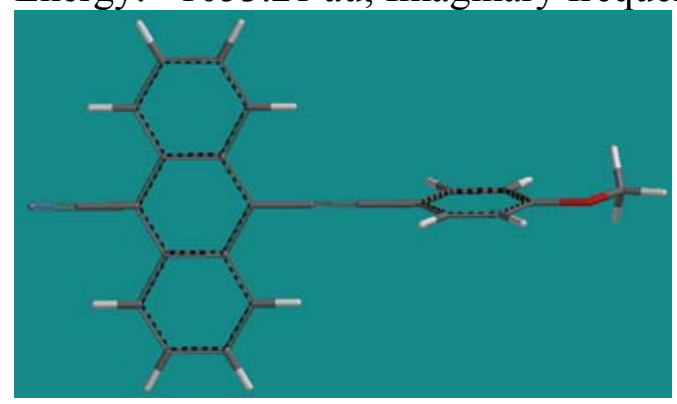

Cartesian Coordinates (Angstroms) Atom $\mathrm{X}$ Y Z

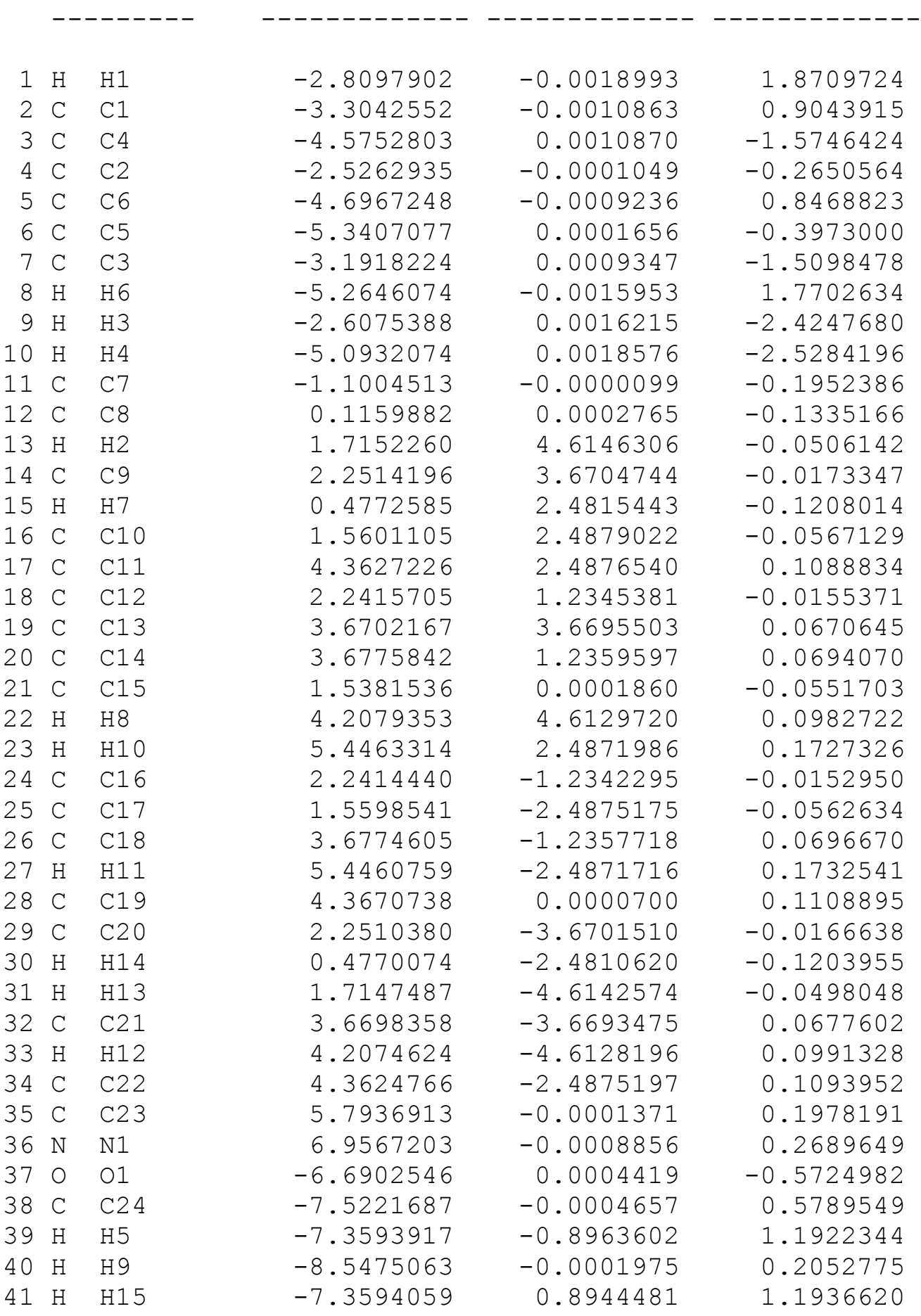


DMA-PEAN

Energy: -1072.96 au; Imaginary frequency: none

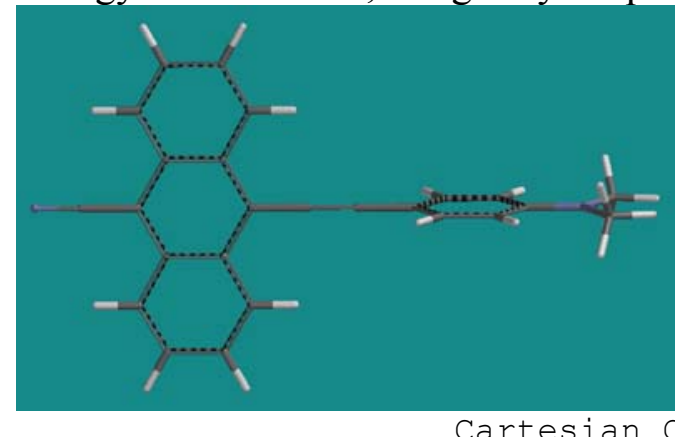

$\mathrm{X}$

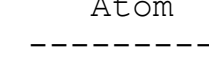

$X$
-------------

$-$

$\begin{array}{lll}1 & \mathrm{H} & \mathrm{H} 1\end{array}$

2 C C1

$3 \mathrm{C} \quad \mathrm{C} 4$

4 C $\mathrm{C} 2$

5 C C 6

6 C C5

$7 \mathrm{C} \mathrm{C} 3$

$8 \mathrm{H} H 6$

$9 \mathrm{H} \quad \mathrm{H} 3$

$10 \mathrm{H} \quad \mathrm{H} 4$

$11 \mathrm{C} \quad \mathrm{C} 7$

$12 \mathrm{C} \quad \mathrm{C} 8$

$13 \mathrm{H} \quad \mathrm{H} 2$

$14 \mathrm{C} \quad \mathrm{C} 9$

$15 \mathrm{H} \quad \mathrm{H} 7$

$16 \mathrm{C} \quad \mathrm{C} 10$

$17 \mathrm{C} \quad \mathrm{C} 11$

$18 \mathrm{C} \quad \mathrm{C} 12$

19 C C13

$20 \mathrm{C} \quad \mathrm{C} 14$

$21 \mathrm{C} \quad \mathrm{C} 15$

$22 \mathrm{H} H 8$

$23 \mathrm{H} \mathrm{H} 1 \mathrm{O}$

$24 \mathrm{C} \quad \mathrm{C} 16$

$25 \mathrm{C} \quad \mathrm{C} 17$

$26 \mathrm{C} \quad \mathrm{C} 18$

$27 \mathrm{H} \quad \mathrm{H} 11$

$28 \mathrm{C} \quad \mathrm{C} 19$

29 C C20

$30 \mathrm{H} \quad \mathrm{H} 14$

$31 \mathrm{H} \quad \mathrm{H} 13$

32 C C21

$33 \mathrm{H} H 12$

34 C C22

$35 \mathrm{C} \quad \mathrm{C} 23$

$36 \mathrm{~N} N 1$

$37 \mathrm{~N}$ N2

38 C C24

$39 \mathrm{H}$ H 9

$40 \mathrm{H}$ H15

$41 \mathrm{H} \quad \mathrm{H} 16$

42 C C25

$43 \mathrm{H}$ H5

$44 \mathrm{H} \quad \mathrm{H} 17$

$45 \mathrm{H}$ H18

0.0000000

0.0000000

0.0000000

0.0000000

0.0000000

0.0000000

0.0000000

0.0000000

0.0000000

0.0000000

0.0000000

0.0000000

$-4.6141606$

$-3.6701174$

$-2.4793837$

$-2.4872062$

$-2.4877355$

$-1.2340860$

$-3.6694622$

$-1.2358121$

0.0000000

$-4.6130317$

$-2.4878000$

1.2340860

2. 4872062

1.2358121

2. 4878000

0.0000000

3.6701174

2.4793837

4.6141606

3.6694622

4.6130317

2.4877355

0.0000000

0.0000000

0.0000000

0.0000000

0.8889325

$-0.8889325$

0.0000000

0.0000000

$-0.8889325$

0.8889325

0.0000000
2.1485457

1. 2036944

$-1.2092562$

0.0000000

1. 2092562

0.0000000

$-1.2036944$

2. 1626840

$-2.1485457$

$-2.1626840$

0.0000000

0.0000000

0.0000000

0.0000000

0.0000000

0.0000000

0.0000000

0.0000000

0.0000000

0.0000000

0.0000000

0.0000000

0.0000000

0.0000000

0.0000000

0.0000000

0.0000000

0.0000000

0.0000000

0.0000000

0.0000000

0.0000000

0.0000000

0.0000000

0.0000000

0.0000000

0.0000000

$-1.2562494$

$-1.8626275$

$-1.8626275$

$-1.0501108$

1. 2562494

1. 8626275

1. 8626275

1. 0501108
(Angstroms)

$\mathrm{Z}$

$$
\begin{aligned}
& 2.0479106 \\
& 2.5834344 \\
& 3.9701128 \\
& 1.8531353 \\
& 3.9701128 \\
& 4.7068041 \\
& 2.5834344 \\
& 4.4842286 \\
& 2.0479106 \\
& 4.4842286 \\
& 0.4274937
\end{aligned}
$$

$-0.7912959$

$-2.3926595$

$-2.9301549$

$-1.1535290$

$-2.2382831$

$-5.0455578$

$-2.9213392$

$-4.3513890$

$-4.3597493$

$-2.2153016$

$-4.8898969$

$-6.1310790$

$-2.9213392$

$-2.2382831$

$-4.3597493$

$-6.1310790$

$-5.0511024$

$-2.9301549$

$-1.1535290$

$-2.3926595$

$-4.3513890$

$-4.8898969$

$-5.0455578$

$-6.4802229$

$-7.6455216$

6.0875948

6.8159521

6.5893544

6.5893544

7.8874989

6.8159521

6.5893544

6.5893544

7.8874989 


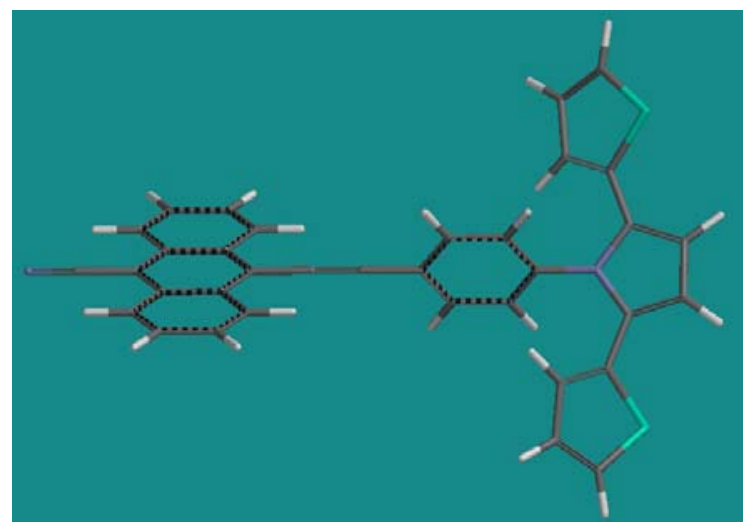

Atom

Cartesian Coordinates (Angstroms) $\mathrm{X}$

Y

Z

\begin{tabular}{|c|c|c|c|c|}
\hline 1 & $\mathrm{H} 1$ & -2.1481883 & 0.1313284 & 0.6030351 \\
\hline 2 & $\mathrm{C} 1$ & -1.2109280 & 0.0722837 & 1.1470052 \\
\hline 3 & $\mathrm{C} 4$ & 1.2094023 & -0.0727361 & 2.5375784 \\
\hline 4 & $\mathrm{C} 2$ & 0.0000000 & 0.0000000 & 0.4306551 \\
\hline 5 & $\mathrm{C} 6$ & -1.2094023 & 0.0727361 & 2.5375784 \\
\hline 6 & $\mathrm{C} 5$ & 0.0000000 & 0.0000000 & 3.2365632 \\
\hline 7 & C3 & 1.2109280 & -0.0722837 & 1.1470052 \\
\hline 8 & $\mathrm{H} 6$ & -2.1412066 & 0.1335732 & 3.0903690 \\
\hline 9 & H3 & 2.1481883 & -0.1313284 & 0.6030351 \\
\hline 10 & H4 & 2.1412066 & -0.1335732 & 3.0903690 \\
\hline 11 & C7 & 0.0000000 & 0.0000000 & -0.9960093 \\
\hline 12 & $\mathrm{C} 8$ & 0.0000000 & 0.0000000 & -2.2134056 \\
\hline 13 & $\mathrm{H} 2$ & -2.2952263 & -4.0047266 & -3.8139198 \\
\hline 14 & C9 & -1.8277730 & -3.1841547 & -4.3507965 \\
\hline 15 & $\mathrm{H} 7$ & -1.2354997 & -2.1556754 & -2.5727614 \\
\hline 16 & $\mathrm{C} 10$ & -1.2404124 & -2.1581664 & -3.6574885 \\
\hline 17 & $\mathrm{C} 11$ & -1.2406501 & -2.1563401 & -6.4654286 \\
\hline 18 & $\mathrm{C} 12$ & -0.6182923 & -1.0697596 & -4.3394977 \\
\hline 19 & C13 & -1.8278780 & -3.1825790 & -5.7724143 \\
\hline 20 & $\mathrm{C} 14$ & -0.6180405 & -1.0708516 & -5.7783124 \\
\hline 21 & C15 & 0.0000000 & 0.0000000 & -3.6367597 \\
\hline 22 & H8 & -2.2960754 & -4.0013965 & -6.3112790 \\
\hline 23 & $\mathrm{H} 10$ & -1.2393597 & -2.1542885 & -7.5509681 \\
\hline 24 & C16 & 0.6182923 & 1.0697596 & -4.3394977 \\
\hline 25 & C17 & 1.2404124 & 2.1581664 & -3.6574885 \\
\hline 26 & $\mathrm{C} 18$ & 0.6180405 & 1.0708516 & -5.7783124 \\
\hline 27 & H11 & 1.2393597 & 2.1542885 & -7.5509681 \\
\hline 28 & C19 & 0.0000000 & 0.0000000 & -6.4684356 \\
\hline 29 & $\mathrm{C} 20$ & 1.8277730 & 3.1841547 & -4.3507965 \\
\hline 30 & $\mathrm{H} 14$ & 1.2354997 & 2.1556754 & -2.5727614 \\
\hline 31 & $\mathrm{H} 13$ & 2.2952263 & 4.0047266 & -3.813919 \\
\hline 32 & $\mathrm{C} 21$ & 1.8278780 & 3.1825790 & -5.772414 \\
\hline 33 & $\mathrm{H} 12$ & 2.2960754 & 4.0013965 & -6.311279 \\
\hline 34 & $\mathrm{C} 22$ & 1.2406501 & 2.1563401 & -6.465428 \\
\hline
\end{tabular}




\begin{tabular}{|c|c|c|c|c|}
\hline $35 \mathrm{C}$ & $\mathrm{C} 23$ & 0.0000000 & 0.0000000 & -7.8981963 \\
\hline $36 \mathrm{~N}$ & $\mathrm{~N} 1$ & 0.0000000 & 0.0000000 & -9.0631859 \\
\hline $37 \mathrm{~N}$ & N2 & 0.0000000 & 0.0000000 & 4.6640753 \\
\hline $38 \mathrm{C}$ & $\mathrm{C} 24$ & 0.3837411 & -1.0709210 & 5.4749141 \\
\hline 39 & $\mathrm{C} 25$ & -0.3837411 & 1.0709210 & 5.4749141 \\
\hline $40 \mathrm{C}$ & $\mathrm{C} 26$ & -0.2378190 & 0.6634217 & 6.7931604 \\
\hline $41 \mathrm{H}$ & H1 5 & -0.4327580 & 1.2842170 & 7.6570071 \\
\hline $42 \mathrm{C}$ & $\mathrm{C} 27$ & 0.2378190 & -0.6634217 & 6.7931604 \\
\hline 43 & H5 & 0.4327580 & -1.2842170 & 7.6570071 \\
\hline 44 & $\mathrm{C} 28$ & -0.8125027 & 2.3650005 & 4.9703085 \\
\hline 45 & $\mathrm{~S} 2$ & -2.0322334 & 3.2642326 & 5.8613924 \\
\hline 46 & C30 & -1.9397226 & 4.5990560 & 4.7578133 \\
\hline 47 & C31 & -1.0411960 & 4.3580204 & 3.7556060 \\
\hline $48 \mathrm{C}$ & C32 & -0.3987674 & 3.0916627 & 3.8744204 \\
\hline $49 \mathrm{H}$ & $\mathrm{H} 2 \mathrm{O}$ & -2.5507193 & 5.4775050 & 4.9165936 \\
\hline $50 \mathrm{H}$ & H21 & -0.8303113 & 5.0645798 & 2.9598903 \\
\hline $51 \mathrm{H}$ & H23 & 0.3607440 & 2.7411712 & 3.1861217 \\
\hline 52 & C33 & 0.8125027 & -2.3650005 & 4.9703085 \\
\hline $53 \mathrm{C}$ & C34 & 0.3987674 & -3.0916627 & 3.8744204 \\
\hline $54 \mathrm{C}$ & C35 & 1.0411960 & -4.3580204 & 3.7556060 \\
\hline $55 \mathrm{C}$ & C36 & 1.9397226 & -4.5990560 & 4.7578133 \\
\hline $56 \mathrm{~S}$ & S 1 & 2.0322334 & -3.2642326 & 5.8613924 \\
\hline 57 & H2 6 & -0.3607440 & -2.7411712 & 3.1861217 \\
\hline $\mathrm{H}$ & H27 & 0.8303113 & -5.0645798 & 2.9598903 \\
\hline $59 \mathrm{H}$ & H3O & 2.5507193 & -5.4775050 & 4.916593 \\
\hline
\end{tabular}

Point Group $=\mathrm{CN}$ Order $=2$ Nsymop $=2$

\section{$\mathbf{A n}_{2} \mathbf{N}$-PEAN Energy: $-1685.35 \mathrm{au}$; imaginary frequency: none}

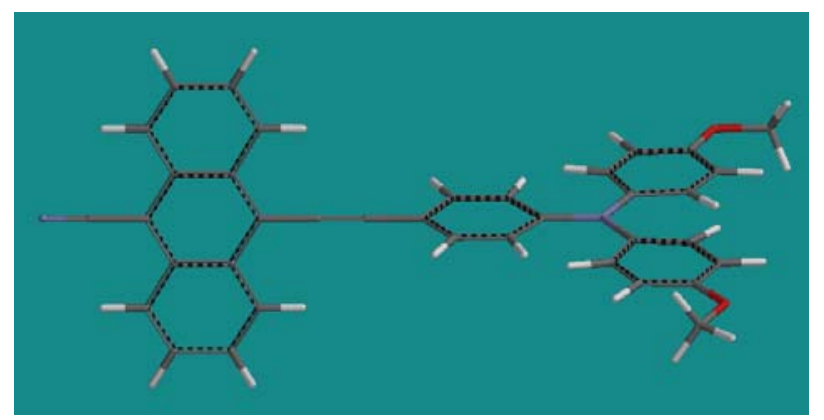

Atom

Cartesian Coordinates (Angstroms)

$\begin{array}{lll}1 & \mathrm{H} & \mathrm{H} 1 \\ 2 & \mathrm{C} & \mathrm{C} 1 \\ 3 & \mathrm{C} & \mathrm{C} 4 \\ 4 & \mathrm{C} & \mathrm{C} 2 \\ 5 & \mathrm{C} & \mathrm{C} 6 \\ 6 & \mathrm{C} & \mathrm{C} 5 \\ 7 & \mathrm{C} & \mathrm{C} 3\end{array}$

$\mathrm{X}$

Y

Z

$\begin{array}{rrr} & & \\ 0.8583758 & -2.0588145 & 0.0000000 \\ 0.3579979 & -1.0947587 & 0.0000000 \\ -1.0515128 & 1.2142336 & 0.0000000 \\ 1.1117545 & 0.0784879 & 0.0000000 \\ -1.0330903 & -1.0938739 & 0.0000000 \\ -1.8821597 & 0.0540516 & 0.0000000 \\ 0.3389038 & 1.2394548 & 0.0000000\end{array}$




\begin{tabular}{|c|c|c|c|c|}
\hline $8 \mathrm{H}$ & H6 & -1.3795554 & -2.0896369 & 0.0000000 \\
\hline $9 \mathrm{H}$ & H3 & 0.8227695 & 2.2118346 & 0.0000000 \\
\hline 10 & $\mathrm{H} 4$ & -1.4185461 & 2.2018418 & 0.0000000 \\
\hline 11 & C7 & 2.5351879 & 0.0905420 & 0.0000000 \\
\hline 12 & $\mathrm{C} 8$ & 3.7529780 & 0.1029547 & 0.0000000 \\
\hline 13 & $\mathrm{H} 2$ & 5.3530836 & 0.1206017 & 4.6146372 \\
\hline $14 \mathrm{C}$ & $\mathrm{Cg}$ & 5.8903992 & 0.1268400 & 3.6705378 \\
\hline 15 & $\mathrm{H} 7$ & 4.1130833 & 0.1060802 & 2.4818872 \\
\hline $16 c$ & $\mathrm{C} 10$ & 5.1978111 & 0.1186635 & 2.4878861 \\
\hline $17 \mathrm{C}$ & $\mathrm{C} 11$ & 8.0058052 & 0.1518378 & 2.4877009 \\
\hline 18 & $\mathrm{C} 12$ & 5.8808114 & 0.1266396 & 1.2344195 \\
\hline $19 \mathrm{C}$ & $\mathrm{C} 13$ & 7.3118731 & 0.1437313 & 3.6696723 \\
\hline 20 & $\mathrm{C} 14$ & 7.3194846 & 0.1436781 & 1.2357576 \\
\hline 210 & C15 & 5.1766801 & 0.1185746 & 0.0000000 \\
\hline $22 \mathrm{H}$ & $\mathrm{H} 8$ & 7.8503182 & 0.1503439 & 4.6131866 \\
\hline $23 \mathrm{H}$ & $\mathrm{H} 10$ & 9.0913591 & 0.1647522 & 2.4866183 \\
\hline $24 c$ & C16 & 5.8808114 & 0.1266396 & -1.2344195 \\
\hline 25 & C17 & 5.1978111 & 0.1186635 & -2.4878861 \\
\hline $26 c$ & $\mathrm{C} 18$ & 7.3194846 & 0.1436781 & -1.2357576 \\
\hline $27 \mathrm{H}$ & $\mathrm{H} 11$ & 9.0913591 & 0.1647522 & -2.4866183 \\
\hline 28 & $\mathrm{C} 19$ & 8.0104108 & 0.1518747 & 0.0000000 \\
\hline 29 & $\mathrm{C} 20$ & 5.8903992 & 0.1268400 & -3.6705378 \\
\hline 30 & $\mathrm{H} 14$ & 4.1130833 & 0.1060802 & -2.4818872 \\
\hline $31 \mathrm{H}$ & $\mathrm{H} 13$ & 5.3530836 & 0.1206017 & -4.6146372 \\
\hline $32 c$ & $\mathrm{C} 21$ & 7.3118731 & 0.1437313 & -3.6696723 \\
\hline 33 & $\mathrm{H} 12$ & 7.8503182 & 0.1503439 & -4.6131866 \\
\hline 34 & $\mathrm{C} 22$ & 8.0058052 & 0.1518378 & -2.4877009 \\
\hline 35 & $\mathrm{C} 23$ & 9.4396162 & 0.1690948 & 0.0000000 \\
\hline $36 N$ & N1 & 10.6046786 & 0.1830222 & 0.0000000 \\
\hline 371 & N2 & -3.3704386 & 0.0483194 & 0.0000000 \\
\hline 38 & $\mathrm{C} 24$ & -4.1347417 & 1.3579194 & 0.0000000 \\
\hline $39 c$ & $\mathrm{C} 25$ & -5.6539299 & 3.9173347 & 0.0000000 \\
\hline $40 \mathrm{C}$ & $\mathrm{C} 26$ & -3.5673399 & 2.6683773 & 0.0000000 \\
\hline 410 & $\mathrm{C} 27$ & -5.5467733 & 1.4903780 & 0.0000000 \\
\hline $42 c$ & $\mathrm{C} 28$ & -6.2801011 & 2.6810991 & 0.0000000 \\
\hline 43 & $\mathrm{C} 29$ & -4.2666063 & 3.8696531 & 0.0000000 \\
\hline $44 \mathrm{H}$ & $\mathrm{H} 5$ & -2.5325564 & 2.8758665 & 0.0000000 \\
\hline $45 \mathrm{H}$ & $\mathrm{H} 9$ & -6.2166385 & 0.6766597 & 0.0000000 \\
\hline 46 & $\mathrm{H} 15$ & -7.3610639 & 2.5908689 & 0.0000000 \\
\hline 47 & $\mathrm{H} 16$ & -3.7018402 & 4.7971452 & 0.0000000 \\
\hline 48 & C30 & -4.1235578 & -1.2671079 & 0.0000000 \\
\hline $49 \mathrm{C}$ & C31 & -5.5991359 & -3.8528862 & 0.0000000 \\
\hline $50 c$ & C32 & -5.5417502 & -1.4220637 & 0.0000000 \\
\hline 51 & C33 & -3.5403283 & -2.5618044 & 0.0000000 \\
\hline 52 & C34 & -4.2148724 & -3.7868180 & 0.0000000 \\
\hline 53 & C35 & -6.2422351 & -2.6227031 & 0.0000000 \\
\hline 54 & $\mathrm{H} 18$ & -6.2301214 & -0.6226232 & 0.0000000 \\
\hline 55 & $\mathrm{H} 19$ & -2.5022366 & -2.7470802 & 0.0000000 \\
\hline 56 & $\mathrm{H} 2 \mathrm{O}$ & -3.6025956 & -4.6819706 & 0.0000000 \\
\hline 57 & H21 & -7.3276705 & -2.5894410 & 0.0000000 \\
\hline 580 & 01 & -6.3624752 & -4.9788561 & 0.0000000 \\
\hline
\end{tabular}




$\begin{array}{rllrrr}59 & \mathrm{O} & \text { O2 } & -6.2564121 & 5.1370283 & 0.0000000 \\ 60 & \mathrm{C} & \mathrm{C} 36 & -5.6894406 & -6.2292122 & 0.0000000 \\ 61 & \mathrm{H} & \mathrm{H} 22 & -5.0645508 & -6.3495518 & -0.8948443 \\ 62 & \mathrm{H} & \mathrm{H} 23 & -6.4704707 & -6.9914792 & 0.0000000 \\ 63 & \mathrm{H} & \mathrm{H} 24 & -5.0645508 & -6.3495518 & 0.8948443 \\ 64 & \mathrm{C} & \mathrm{C} 37 & -7.6760418 & 5.1676204 & 0.0000000 \\ 65 & \mathrm{H} & \mathrm{H} 17 & -8.0889299 & 4.6833436 & -0.8947973 \\ 66 & \mathrm{H} & \mathrm{H} 25 & -7.9542106 & 6.2229720 & 0.0000000 \\ 67 & \mathrm{H} & \mathrm{H} 26 & -8.0889299 & 4.6833436 & 0.8947973\end{array}$

Point Group $=\mathrm{CS}$ Order $=1$ Nsymop $=2$

DTP-PAN $^{\bullet}$ (radical anion) Energy: - 21.75.46 au; imaginary frequency: none

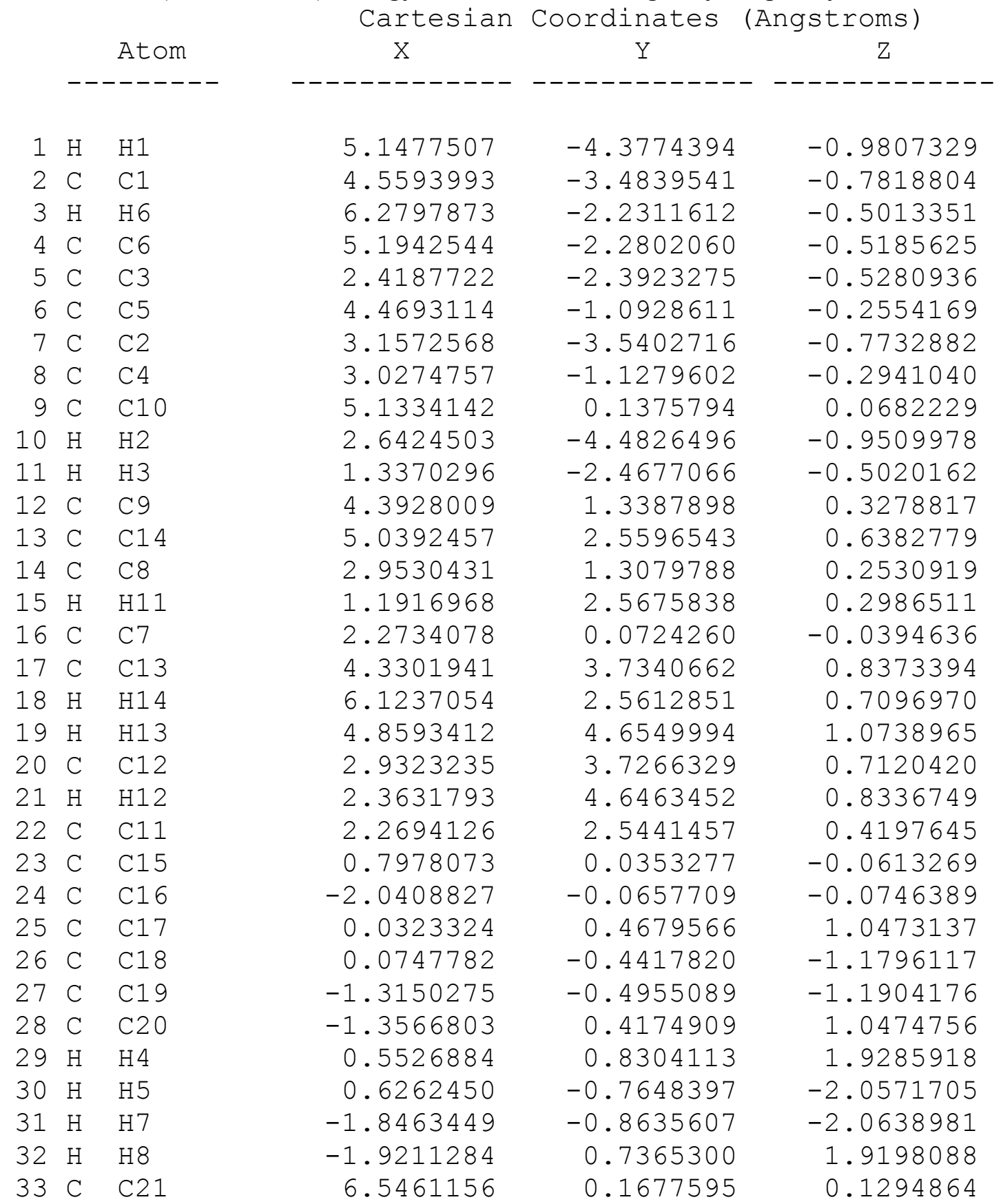




\begin{tabular}{|c|c|c|c|c|}
\hline 341 & N1 & 7.7172616 & 0.1934025 & 0.1810694 \\
\hline 351 & N2 & -3.4748177 & -0.1471918 & -0.0646688 \\
\hline 36 & $\mathrm{C} 22$ & -4.2201618 & -1.3125898 & 0.0953695 \\
\hline 37 & $\mathrm{C} 23$ & -5.5651802 & -0.9552695 & 0.0668797 \\
\hline 38 & $\mathrm{C} 27$ & -4.3435132 & 0.9320067 & -0.1880547 \\
\hline 39 & $\mathrm{C} 28$ & -5.6421427 & 0.4375812 & -0.1116045 \\
\hline 40 & H3 4 & -6.3917446 & -1.6438982 & 0.1807608 \\
\hline 41 & H35 & -6.5376270 & 1.0366882 & -0.2058474 \\
\hline 42 & $\mathrm{C} 24$ & -3.9506587 & 2.3194728 & -0.3688102 \\
\hline 43 & $\mathrm{C} 25$ & -4.6773222 & 3.4169621 & 0.0482356 \\
\hline 44 & $\mathrm{C} 26$ & -4.1218387 & 4.6622579 & -0.3578353 \\
\hline 45 & $\mathrm{C} 29$ & -2.9705776 & 4.5156253 & -1.0827367 \\
\hline 46 & $\mathrm{~S} 2$ & -2.5448528 & 2.8465197 & -1.2830934 \\
\hline 47 & H1 5 & -5.5755816 & 3.3217145 & 0.6488399 \\
\hline 48 & $\mathrm{H} 16$ & -4.5501237 & 5.6256794 & -0.1004097 \\
\hline 49 & H19 & -2.3220845 & 5.2861332 & -1.4784536 \\
\hline 50 & C31 & -3.6532981 & -2.6392142 & 0.2681903 \\
\hline 51 & C32 & -2.4490456 & -3.0631545 & 0.7888358 \\
\hline 52 & C33 & -2.3046668 & -4.4811002 & 0.8113242 \\
\hline 53 & C34 & -3.3905796 & -5.1431177 & 0.3112856 \\
\hline 54 & S 1 & -4.6159466 & -4.0328959 & -0.2207120 \\
\hline $\mathrm{H}$ & H2 4 & -1.6933140 & -2.3830652 & 1.1594147 \\
\hline 56 & H2 6 & -1.4208146 & -4.9820411 & 1.1923646 \\
\hline $\mathrm{H}$ & H2 8 & -3.5465249 & -6.2094688 & 0.2173947 \\
\hline
\end{tabular}

Point Group $=\mathrm{C} 1$ Order $=1$ Nsymop $=1$

DTP-PAN $^{+\bullet}$ (radical cation) Energy: -21.75 .19 au; imaginary frequency: none

\begin{tabular}{|c|c|c|c|c|c|}
\hline \multirow{2}{*}{\multicolumn{3}{|c|}{ Atom }} & \multirow{2}{*}{$\begin{array}{l}\text { Cartesian } \\
\quad \mathrm{X}\end{array}$} & \multicolumn{2}{|c|}{$\begin{array}{c}\text { Coordinates } \\
\mathrm{Y}\end{array}$} \\
\hline & & & & ---- & ----- \\
\hline 1 & $\mathrm{H}$ & $\mathrm{H} 1$ & 5.1987971 & -4.2156213 & -1.4219950 \\
\hline 2 & $\mathrm{C}$ & $\mathrm{C} 1$ & 4.6162859 & -3.3482421 & -1.1268246 \\
\hline 3 & $\mathrm{H}$ & $\mathrm{H} 6$ & 6.3298475 & -2.1163082 & -0.8017214 \\
\hline 4 & $\mathrm{C}$ & $\mathrm{C} 6$ & 5.2466794 & -2.1820810 & -0.7840398 \\
\hline 5 & $\mathrm{C}$ & C3 & 2.4469139 & -2.3445282 & -0.7208558 \\
\hline 6 & C & $\mathrm{C} 5$ & 4.4990389 & -1.0297985 & -0.3938938 \\
\hline 7 & $\mathrm{C}$ & $\mathrm{C} 2$ & 3.1977098 & -3.4300133 & -1.0881334 \\
\hline 8 & $\mathrm{C}$ & $\mathrm{C} 4$ & 3.0574958 & -1.0961949 & -0.3734071 \\
\hline 9 & $\mathrm{C}$ & $\mathrm{C} 10$ & 5.1356061 & 0.1753825 & -0.0210417 \\
\hline 10 & $\mathrm{H}$ & $\mathrm{H} 2$ & 2.7081032 & -4.3640290 & -1.3490722 \\
\hline 11 & $\mathrm{H}$ & H3 & 1.3666837 & -2.4301149 & -0.6877443 \\
\hline 12 & $\mathrm{C}$ & C9 & 4.3973795 & 1.3171805 & 0.3637855 \\
\hline 13 & $\mathrm{C}$ & $\mathrm{C} 14$ & 5.0423611 & 2.5345032 & 0.7389715 \\
\hline 14 & $\mathrm{C}$ & $\mathrm{C} 8$ & 2.9555938 & 1.2515344 & 0.3680045 \\
\hline 15 & $\mathrm{H}$ & $\mathrm{H} 11$ & 1.1544083 & 2.4248169 & 0.7072206 \\
\hline 16 & $\mathrm{C}$ & $\mathrm{C} 7$ & 2.3086837 & 0.0452882 & 0.0033127 \\
\hline 17 & $\mathrm{C}$ & C13 & 4.3132524 & 3.6387302 & 1.0909841 \\
\hline
\end{tabular}




\begin{tabular}{|c|c|c|c|c|}
\hline $18 \mathrm{H}$ & $\mathrm{H} 14$ & 6.1271351 & 2.5694577 & 0.7371799 \\
\hline $19 \mathrm{H}$ & H13 & 4.8190252 & 4.5566308 & 1.3747832 \\
\hline 20 & C12 & 2.8926842 & 3.5902435 & 1.0764756 \\
\hline $21 \mathrm{H}$ & $\mathrm{H} 12$ & 2.3243847 & 4.4765507 & 1.3442624 \\
\hline 22 & C11 & 2.2382081 & 2.4395649 & 0.7237058 \\
\hline 23 & C15 & 0.8159496 & -0.0176688 & 0.0092675 \\
\hline 24 & C16 & -1.9862932 & -0.0979975 & 0.0145701 \\
\hline 25 & C17 & 0.0958174 & 0.0683771 & 1.2139969 \\
\hline 26 & C18 & 0.0981826 & -0.1547996 & -1.1912772 \\
\hline 27 & C19 & -1.2947628 & -0.2015111 & -1.1951555 \\
\hline 28 & $\mathrm{C} 20$ & -1.2972665 & 0.0347562 & 1.2232092 \\
\hline $29 \mathrm{H}$ & H4 & 0.6352834 & 0.1680948 & 2.1508364 \\
\hline $30 \mathrm{H}$ & H5 & 0.6386738 & -0.2228399 & -2.1302666 \\
\hline 31 & H7 & -1.8416624 & -0.3090600 & -2.1272147 \\
\hline 32 & H8 & -1.8463585 & 0.1057537 & 2.1575053 \\
\hline 33 & C21 & 6.5653320 & 0.2414023 & -0.0341166 \\
\hline $34 \mathrm{~N}$ & N1 & 7.7281168 & 0.2955292 & -0.0450002 \\
\hline $35 \mathrm{~N}$ & N2 & -3.4274587 & -0.1380890 & 0.0167058 \\
\hline 36 & $\mathrm{C} 22$ & -4.2088737 & -1.2773606 & 0.1760851 \\
\hline 37 & $\mathrm{C} 23$ & -5.5722979 & -0.8625425 & 0.1019481 \\
\hline 38 & $\mathrm{C} 27$ & -4.2547515 & 0.9637880 & -0.1537384 \\
\hline 39 & $\mathrm{C} 28$ & -5.6018559 & 0.4920211 & -0.0983416 \\
\hline 40 & H34 & -6.4258193 & -1.5199935 & 0.1925916 \\
\hline 41 & H35 & -6.4817386 & 1.1106176 & -0.1954915 \\
\hline 42 & C24 & -3.8609365 & 2.3151934 & -0.3454773 \\
\hline 43 & C25 & -4.7655207 & 3.3742197 & -0.5250980 \\
\hline 44 & $\mathrm{C} 26$ & -4.1489083 & 4.6230615 & -0.6914137 \\
\hline 45 & C29 & -2.7721970 & 4.5386848 & -0.6404988 \\
\hline 46 & S2 & -2.2085092 & 2.9304263 & -0.3914053 \\
\hline 47 & H1 5 & -5.8396542 & 3.2359468 & -0.5374726 \\
\hline 48 & H1 6 & -4.6867451 & 5.5512610 & -0.8438375 \\
\hline 49 & H19 & -2.0558583 & 5.3445078 & -0.7408967 \\
\hline 50 & C31 & -3.7429629 & -2.6061445 & 0.3743633 \\
\hline 51 & C32 & -2.4539267 & -3.1447909 & 0.4730675 \\
\hline 52 & C33 & -2.4447713 & -4.5358293 & 0.6769223 \\
\hline 53 & C34 & -3.7128792 & -5.0781753 & 0.7348773 \\
\hline 54 & S1 & -4.9430190 & -3.8960723 & 0.5418377 \\
\hline 55 & H2 4 & -1.5535541 & -2.5523704 & 0.405529 \\
\hline 56 & H2 6 & -1.5414868 & -5.1254797 & 0.780691 \\
\hline $57 \mathrm{H}$ & H2 8 & -3.9835648 & -6.1158688 & 0.882740 \\
\hline
\end{tabular}

Point Group $=\mathrm{C} 1$ Order $=1$ Nsymop $=1$ 
DTP-PEAN $^{-\bullet}$ (radical anion) Energy: -2251.64 au; imaginary frequency: none

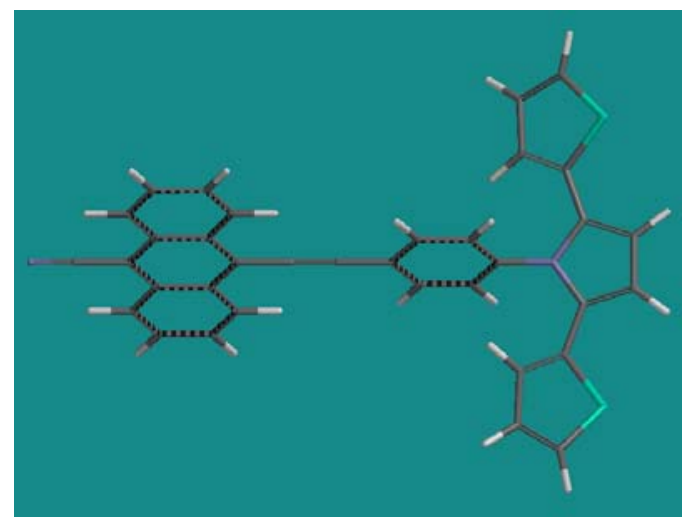

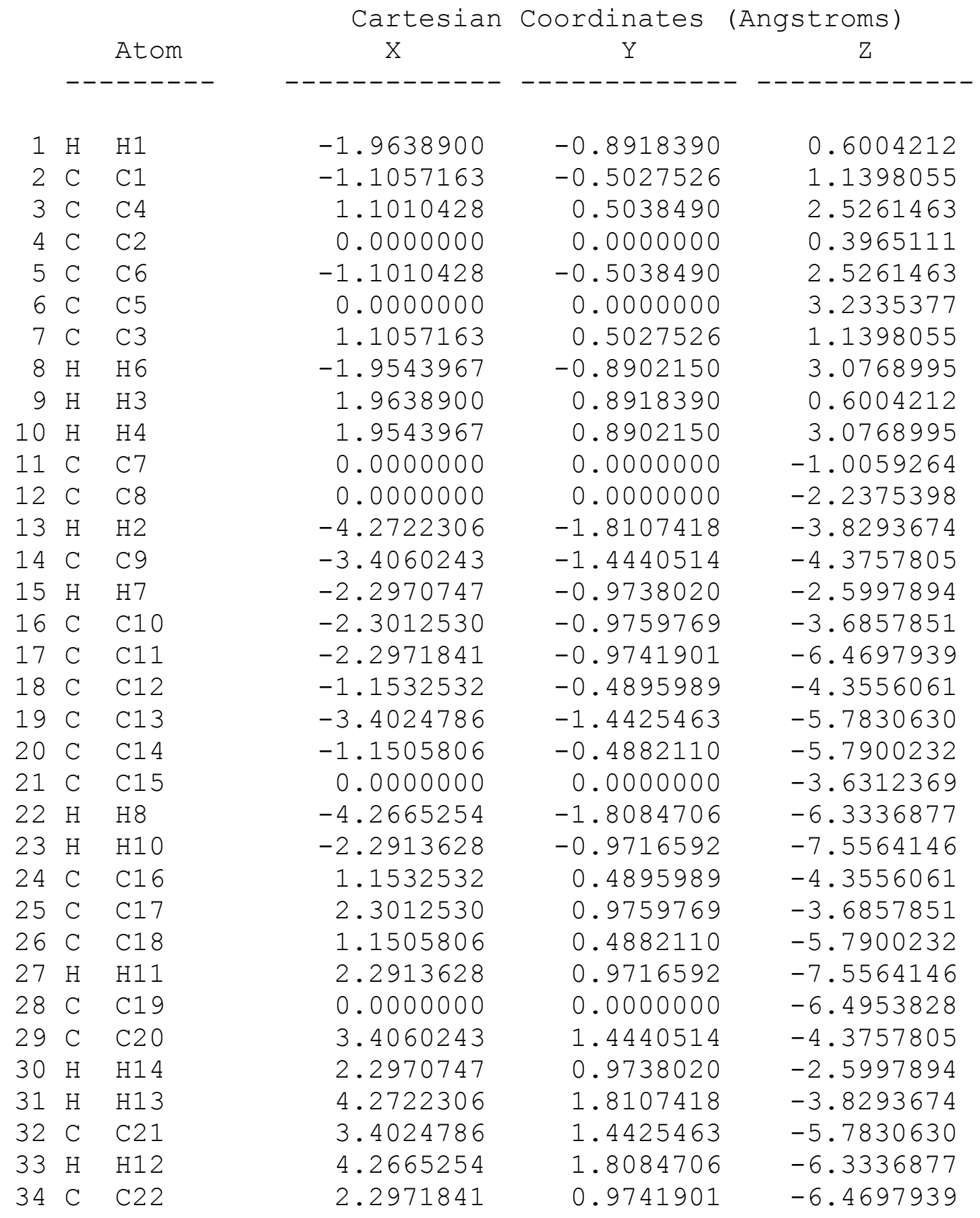




\begin{tabular}{|c|c|c|c|c|}
\hline 35 & $\mathrm{C} 23$ & 0.0000000 & 0.0000000 & -7.9103584 \\
\hline 36 & N1 & 0.0000000 & 0.0000000 & -9.0821358 \\
\hline 37 & N2 & 0.0000000 & 0.0000000 & 4.6684860 \\
\hline 38 & C24 & 0.7448374 & -0.8535129 & 5.4790478 \\
\hline 39 & $\mathrm{C} 25$ & -0.7448374 & 0.8535129 & 5.4790478 \\
\hline 40 & $\mathrm{C} 26$ & -0.4623892 & 0.5294434 & 6.8025028 \\
\hline 41 & H1 5 & -0.8752187 & 1.0329208 & 7.6664495 \\
\hline 42 & $\mathrm{C} 27$ & 0.4623892 & -0.5294434 & 6.8025028 \\
\hline 43 & H5 & 0.8752187 & -1.0329208 & 7.6664495 \\
\hline 44 & $\mathrm{C} 28$ & -1.6333604 & 1.8930093 & 4.989237 \\
\hline 45 & $\mathrm{~S} 2$ & -2.9796616 & 2.3935700 & 6.0102773 \\
\hline 46 & $\mathrm{C} 30$ & -3.4742123 & 3.6060969 & 4.8696440 \\
\hline 47 & C31 & -2.6689734 & 3.6059834 & 3.765216 \\
\hline 48 & C32 & -1.6249264 & 2.6375019 & 3.8288007 \\
\hline 49 & $\mathrm{H} 2 \mathrm{O}$ & -4.3279210 & 4.2355618 & 5.082071 \\
\hline 50 & H2 1 & -2.8052196 & 4.2793833 & 2.925338 \\
\hline 51 & $\mathrm{H} 23$ & -0.8859134 & 2.5049572 & 3.049475 \\
\hline 52 & C33 & 1.6333604 & -1.8930093 & 4.989237 \\
\hline 53 & C34 & 1.6249264 & -2.6375019 & 3.8288007 \\
\hline 54 & C35 & 2.6689734 & -3.6059834 & 3.765216 \\
\hline 55 & C36 & 3.4742123 & -3.6060969 & 4.869644 \\
\hline 56 & S1 & 2.9796616 & -2.3935700 & 6.010277 \\
\hline 57 & H2 6 & 0.8859134 & -2.5049572 & 3.049475 \\
\hline 58 & H2 7 & 2.8052196 & -4.2793833 & 2.925338 \\
\hline 59 & H3O & 4.3279210 & -4.2355618 & 5.082071 \\
\hline
\end{tabular}

Point Group $=\mathrm{CN}$ Order $=2$ Nsymop $=2$ 
$\underline{\text { UV-vis. Absorption solvatochromic spectra of selected compounds }}$
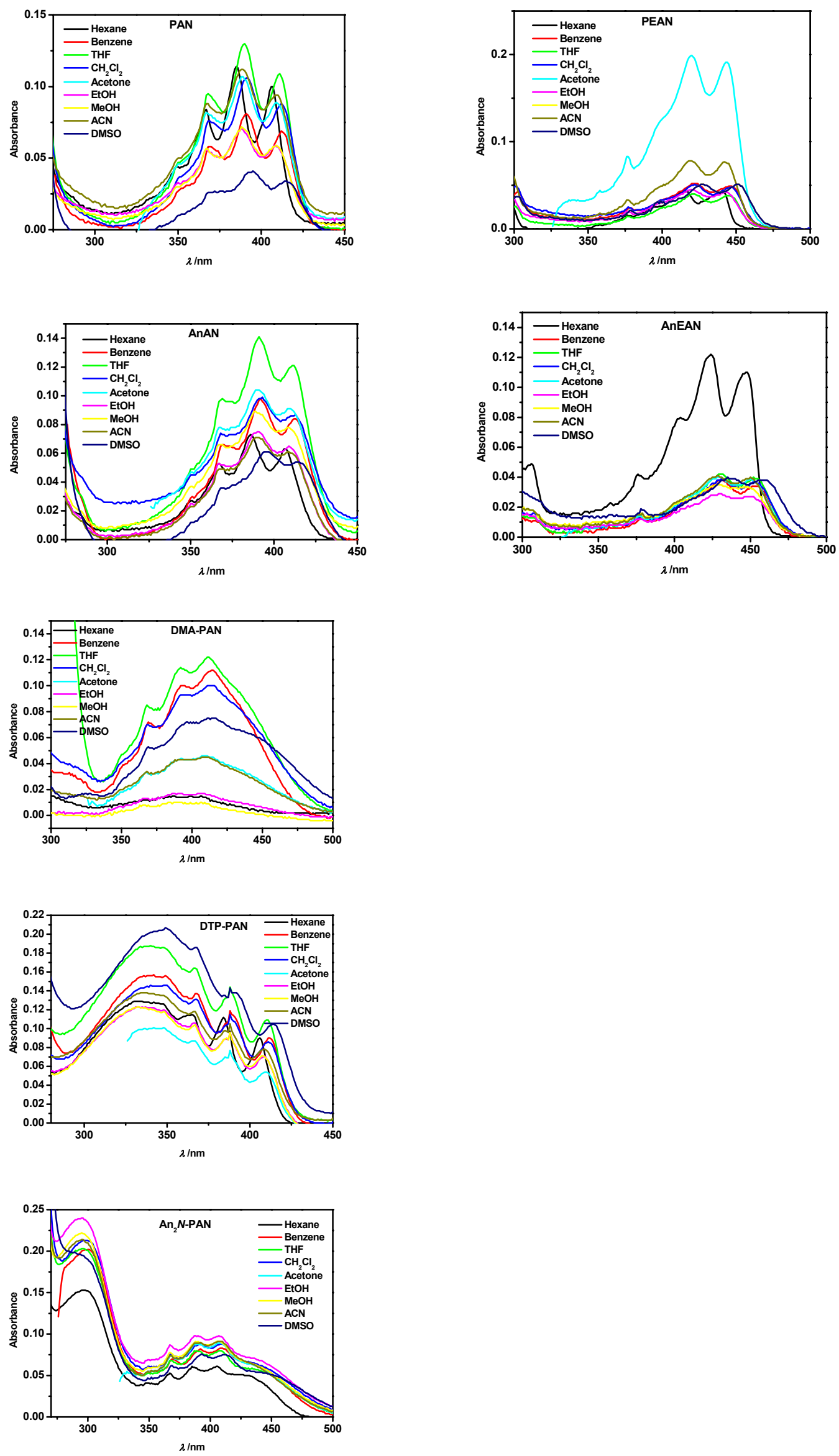


\section{REFERENCES}

1. (a) Nakazaki, J.; Chung, I.; Matsushita, M. M.; Sugawara, T.; Watanabe, R.; Izuoka, A.; Kawada, Y. J. Mater. Chem. 2003, 13, 1011. (b) Kim, K.-S.; Kang, M.-S.; Ma. H.; Jen, A. K.-Y. Chem. Mater. 2004, 16, 1558.

2. (b) Lambert, C.; Nöll. G.; Schmälzlin, E.; Meerholz, K.; Bräuchele, C. Chem. Eur. J. 1998, 4, 2129.

3. Elangovan, A.; Wang, Y.-H.; Ho, T.-I. Org. Lett. 2003, 5, 1841.

4. Reynolds G. A.; Drexhage, K. H. Optics Commun. 1975, 13, 222.

5. (a) Lakovickz, J. R. Principles of Fluorescence Spectroscopy Plenum Press: New York, 1983, p.187 (b) Lippert, Von E. Z. Electrochem.1957, 61, 962.

6. (a) Chen, F.-C.; Ho, J.-H.; Chen, C.-Y.; Su, Y. O.; Ho, T.-I. J. Electroanal. Chem. 2001, 499, 17. (b) Elangovan, A.; Chen, T.-Y.; Chen, C.-Y.; Ho, T.-I. Chem. Commun. 2003, 2146.

7. Elangovan, A.; Kao, K.-M.; Yang, S.-W.; Chen, Y.-L.; Ho, T.-I.; Su, O. Y. J. Org. Chem. 2005, 70, 4460.

8. Spartan'04 Windows, Wavefunction, Inc. 18401 Von Karman Avenue, Suite 370, Irvine, CA 92612 U.S.A.

9. (a) Hehre, W. J. A guide to Molecular Mechanics and Quantum Chemical Calculations, Wavefunction Inc. Irvine, 2003. (b) Lee, C.; Yang, W.; Parr, R. G. Phys. Rev. B 1988, 37, 785. (c) Beck, A. D. J. Chem. Phys. 1993, 98, 5648. 